\title{
Klimawandel - Auswirkungen mit Blick auf den Tourismus
}

\author{
Coordinating Lead Authors (CLAs) \\ Marc Olefs, Herbert Formayer \\ Lead Authors (LAs) \\ Marc Olefs, Herbert Formayer, Andreas Gobiet, Thomas Marke, \\ Wolfgang Schöner \\ Contributing Authors (CAs) \\ Andrea Fischer, Günther Aigner
}

\subsection{Einführung}

Das Klimasystem der Erde besteht aus dem komplexen $\mathrm{Zu}$ sammenspiel der verschiedenen Sphären, bei dem Energieund Stoffströme zwischen den Weltmeeren, den Landmassen und der Atmosphäre ausgetauscht werden. Dieser Austausch wird durch die Energie der Sonne angetrieben, wobei die Energieumsetzung noch zusätzlich durch Wechselwirkungen mit der Erdoberfläche (z. B. Eis, Schnee und Wasser) sowie den Lebewesen modifiziert wird. Grundsätzlich versucht das Klimasystem ein Gleichgewicht - sowohl über Zeit als auch Raum - der Energieverteilung auf der Erde herzustellen. Durch die unterschiedlichen Reaktionszeiten der verschiedenen Sphären kommt es jedoch zu Überlagerungen von verschiedenen Prozessen und periodischen Schwankungen (Stephenson et al. 2004).

Auf kurzen und langen Zeiträumen, von Jahrzehnten bis Millionen von Jahren, finden permanent klimatische Veränderungen auf der Erde statt (Zachos et al. 2001). Geologisch gesehen befindet sich die Erde in einem Eiszeitalter (dem Quartär), da große Flächen (ca. 10 \%) permanent mit Eis oder Schnee bedeckt sind (Ehlers und Gibbard 2011). Während eines Eiszeitalters ist das Klimasystem besonders empfindlich und bereits geringe Schwankungen des Energieeintrages, wie sie etwa durch Veränderungen der Erdbahngeometrie, den sogenannten Milankovitch-Zyklen (Milankovitch 1941), ausgelöst werden, führen zu starken Schwankungen der globalen Mitteltemperatur. Die lang anhaltenden Schwankungen des Strahlungseintrages werden durch positive Rückkopplungen (z. B. Eisalbedo ${ }^{1}$ oder Löslichkeit von Gasen in den Ozeanen) verstärkt und haben zur Entstehung von Kaltzeiten (Glazialen) und Warmzeiten

\footnotetext{
${ }^{1}$ Eisalbedo bezeichnet die Rückstrahlung der vereisten Flächen an den Polkappen.
}

(Interglazialen) geführt (Shakun et al. 2012; Ganopolski et al. 2010). Außerhalb von Eiszeitaltern ist die Wirkung der Milankovitch-Zyklen nicht so ausgeprägt, da die Verstärkung durch die Eisalbedorückkopplung fehlt.

Die derzeitige Warmzeit (Holozän) begann vor etwa 11.000 Jahren und seither ist das Klima der Erde sehr stabil; die globale Mitteltemperatur schwankte nur um weniger als $1^{\circ} \mathrm{C}$ (Walker et al. 2009). Aufgrund der Milankovitch-Zyklen wurde der wärmste Abschnitt vor 7000-4000 Jahren erreicht, wobei sich die Erde aufgrund der Veränderungen der Erdbahngeometrie gerade langsam wieder auf eine neue Kaltzeit zubewegt. Dieser Prozess würde aber noch einige Zehntausende Jahre benötigen, bevor wieder eine Vereisung und starke Abkühlung einsetzen (Tzedakis et al. 2012). Derzeit sprechen immer mehr Wissenschaftler wegen des prägenden Einflusses des Menschen auf den Planeten Erde von einer neuen geologischen Epoche, dem Anthropozän (Waters et al. 2016).

Besonders seit dem Beginn der industriellen Revolution im 18. Jahrhundert greift der Mensch, v. a. durch die Verbrennung fossiler Brennstoffe und die damit einhergehende Freisetzung von Treibhausgasen, wie dem Kohlendioxid $\left(\mathrm{CO}_{2}\right)$, massiv in das Klimasystem ein. Treibhausgase sind in der Lage, die langwellige Wärmestrahlung der Erde zu absorbieren und diese teilweise wieder zum Erdboden zurückzustrahlen. Dadurch erhöht sich die atmosphärische Gegenstrahlung und damit der Energieeintrag zur Erdoberfläche und den bodennahen Luftschichten. Derzeit beträgt der zusätzliche durch menschliche Aktivitäten erhöhte Strahlungseintrag in etwa $2,5 \mathrm{~W} / \mathrm{m}^{2}$ verglichen mit der Zeit vor der industriellen Revolution (Jahr 1750; IPCC 2013, 2018).

Laut dem 1,5-Grad-Bericht des Weltklimarats IPCC (IPCC 2018) liegt die globale Mitteltemperatur heute bereits $1{ }^{\circ} \mathrm{C}$ über dem vorindustriellen Niveau (Zeitraum 1850-1900). Besonders die rasche Erwärmung der letzten Jahrzehnte (Abb. 2.1, links) kann dabei nur durch die menschlichen Ak- 

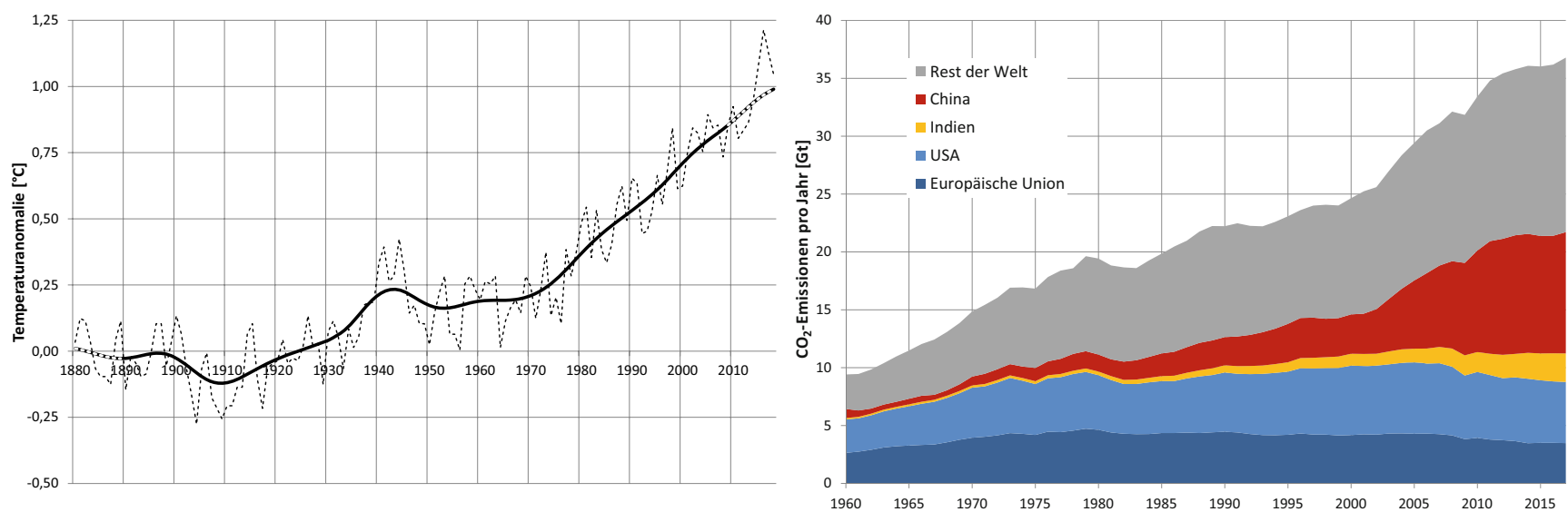

Abb. 2.1 Entwicklung der globalen Mitteltemperatur (Lufttemperatur in $2 \mathrm{~m}$ Höhe) seit 1850, dargestellt als Abweichung (Anomalie) bezogen auf den Zeitraum 1850-1900 (links; Datenquelle: CRU). Im Vergleich dazu die globalen $\mathrm{CO}_{2}$-Emissionen aus fossiler Energie seit 1960 (rechts; Datenquelle: Global Carbon Project). (Grafik: Herbert Formayer)

tivitäten erklärt werden. Die globalen Emissionen an $\mathrm{CO}_{2}$ aus fossiler Energie steigen noch weiter an (Abb. 2.1, rechts). Zwar ist es in Europa und den USA inzwischen gelungen die Emissionen leicht zu reduzieren, jedoch führt die starke wirtschaftliche Entwicklung in Asien und anderen Entwicklungs- und Schwellenländern zu einem weiteren Anstieg der globalen Emissionen.

Der vom Menschen verursachte Klimawandel bezieht sich nicht nur auf die Temperatur. Weltweit kommt es auch zu Änderungen des Niederschlags, der Sonneneinstrahlung und anderer meteorologischer Größen. Die Veränderungen sind jedoch regional sehr unterschiedlich ausgeprägt. Neben den mittleren Verhältnissen ändern sich auch die Extremereignisse (IPCC 2013). Bei der Temperatur muss man generell von einer Zunahme warmer Extreme sowie einer Abnahme kalter Extreme ausgehen (IPCC 2013). Beim Niederschlag ist von einer Zunahme der Niederschlagsintensität auszugehen, da eine wärmere Atmosphäre mehr Wasserdampf aufnehmen und anschließend wieder als Niederschlag abgeben kann (siehe z. B. Gobiet et al. 2014).

Im Pariser Klimaabkommen (UNFCCC 2015) hat sich die Weltgemeinschaft verpflichtet, die globale Erwärmung bis Ende dieses Jahrhunderts auf einem Niveau wesentlich unter $2{ }^{\circ} \mathrm{C}$, wenn möglich auf $1,5^{\circ} \mathrm{C}$, bezogen auf das vorindustrielle Temperaturniveau, zu begrenzen. Um das 1,5-GradZiel zu erreichen, müssen die globalen Emissionen möglichst sofort abnehmen, sodass die Nettoemissionen bereits 2055 auf null gesunken sind. Derartige Emissionsreduktionen können nur durch eine umfassende Neuausrichtung unserer technischen und wirtschaftlichen Entwicklung erreicht werden. Daher spricht man auch von einer notwendigen Transformation der Gesellschaft (Geels 2018).

Um durch Klimamodellierungen die ganze Bandbreite der möglichen zukünftigen Klimaveränderungen zu untersuchen, werden verschiedene Entwicklungen des menschlichen Verhaltens angenommen. Derzeit werden vier verschiedene sogenannte Representative Concentration Pathways (RCPs; van Vuuren et al. 2011; Rogelj et al. 2018) als Rahmenbedingungen in der Klimamodellierung eingesetzt. Jedes RCP hat als Angabe eine Zahl, welche den zusätzlichen Strahlungsantrieb am Ende des 21. Jahrhunderts angibt, verglichen mit dem Niveau vor der industriellen Revolution. Je höher diese Zahl ist, umso stärker ist die menschliche Beeinflussung des Klimas. Das Szenario mit den geringsten Emissionen - in dem das Pariser Abkommen mit großer Wahrscheinlichkeit eingehalten würde - ist das RCP 2.6. Bei diesem Szenario ist am Ende des 21. Jahrhunderts die Treibhausgaswirkung wieder etwa so hoch, wie sie heute bereits ist. Dies bedeutet, dass alle Emissionen, die von jetzt an in die Atmosphäre eingetragen werden, durch natürliche Abbauprozesse oder technische Maßnahmen bis zum Ende des Jahrhunderts wieder aus der Atmosphäre entfernt werden müssten. Das Szenario RCP 8.5 dagegen stellt das Extremszenario dar, bei dem es nicht gelingt, weltweite Klimaschutzziele umzusetzen, und fossile Brennstoffe die Hauptenergiequelle im 21. Jahrhundert bleiben. Zwischen diesen beiden Extremszenarien liegen die Szenarien RCP 4.5 sowie RCP 6. Für diese vier Emissionsszenarien liegen Klimamodellsimulationen vor, wobei für sozioökonomische Untersuchungen noch weitere Emissionsszenarien verwendet werden (Rogelj et al. 2018), auf die hier nicht weiter eingegangen wird. In Abschn. 2.2.2 werden die Ergebnisse der RCPs 2.6, 4.5 und 8.5 für Österreich näher betrachtet.

Im alltäglichen Leben sind die Folgen des Klimawandels, abgesehen von Extremereignissen, die in der öffentlichen Diskussion meist dem Klimawandel zugeschrieben werden, oft schwer wahrnehmbar. Dies gilt auch für Klimagrößen, die für touristische Aktivitäten relevant sind. In der menschlichen 


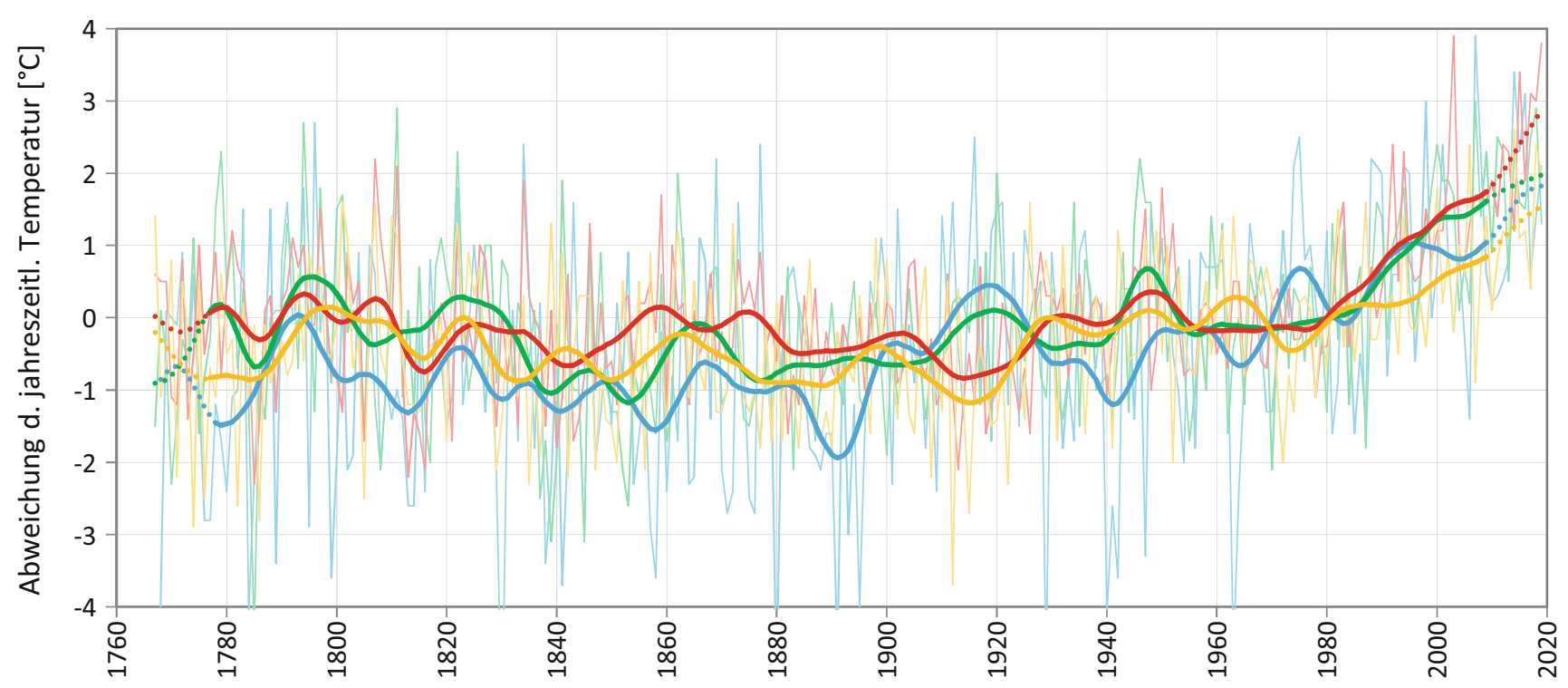

Abb. 2.2 Entwicklung der mittleren Winter- (blau), Frühlings- (grün), Sommer- (rot) und Herbsttemperatur (gelb) in Österreich $1767-2018$. Dargestellt sind jährliche Abweichungen vom Mittel der Jahre 1961-1990 (dünne Linien) und deren geglättete Trends (dicke Linien, 21-jähriger Gauß'scher Tiefpassfilter). (Olefs et al. 2019)

Abb. 2.3 Wintertemperaturen (DJF) der Station Sonnblick

(3105 m) zwischen 1930/1931 und 2019/2020. Linearer Trend über die gesamte Periode (fett, schwarz) und über die 30-jährigen Teilperioden A, B und $\mathrm{C}$ (farbig) mit Angabe des jeweiligen Mittelwertes und des sog. p-Wertes zur Prüfung von etwaigen Trends ( $p$-Werte kleiner 0,05 würden beim gewählten Signifikanzniveau einen statistisch signifikanten Trend bedeuten; Gobiet et al. 2017; aktualisiert). Die Abbildung verdeutlicht damit, dass die Betrachtung kurzer Zeiträume zu falschen Schlussfolgerungen führen kann

\section{Wintertemperaturen Sonnblick (3105 m) der letzten 90 Jahre}

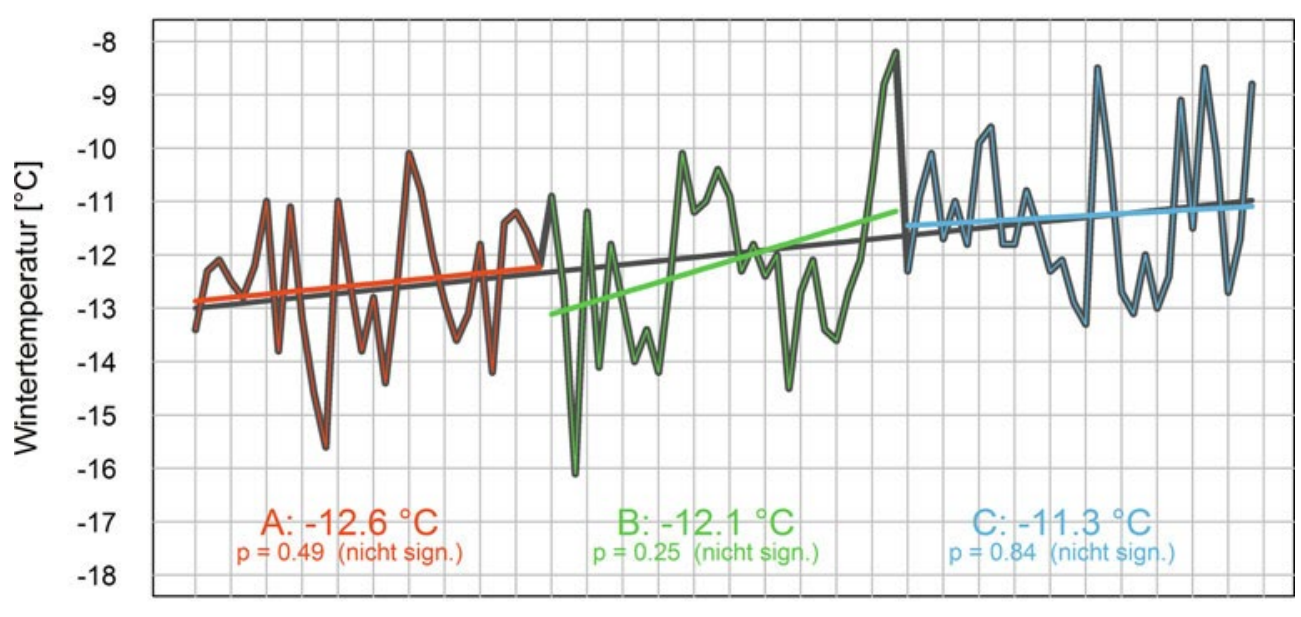

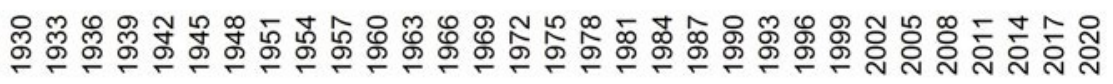
Jahr
Wahrnehmung dominieren vorwiegend die Schwankungen von Jahr zu Jahr gegenüber den langfristigen Änderungen (Abb. 2.2 und 2.3). Dies wird sofort verständlich, wenn man überlegt, dass der Unterschied zwischen einem sehr kalten und einem sehr heißen Sommer etwa $6{ }^{\circ} \mathrm{C}$ beträgt (ZAMG 2019). Im Winter ist dieser Unterschied mit ca. $10^{\circ} \mathrm{C}$ sogar noch größer (ZAMG 2020a). Vergleicht man dies mit dem langfristigen Temperaturanstieg, der in der Größenordnung von $0,2-0,5^{\circ} \mathrm{C}$ pro Dekade liegt, so wird klar, dass dieser bei Betrachtung kurzer Zeiträume daher schwer wahrnehmbar ist. Beim Niederschlag sind Änderungen noch schwieriger erkennbar und daher oft auch nicht statistisch signifikant. Werden jedoch Grenzwerte des Klimas überschritten, etwa kritische Temperaturen für das Pflanzenwachstum, dann wird die Folge des Klimawandels klar sichtbar. Ein Beispiel dafür ist die Verschiebung der Vegetationsperiode. So hat sich beispielsweise der mittlere Termin der Apfelblüte in Österreich in den letzten 40 Jahren um rund 14 Tage nach vorne verschoben (Kromp-Kolb und Formayer 2018). Auch der markante Gletscherrückgang im Alpenraum (Fischer et al. 2015; Helf- 
richt et al. 2019) macht den Klimawandel für uns Menschen direkt erlebbar.

Trotz der globalen Erwärmung wird es auch in Zukunft immer wieder kühle oder verregnete Sommer geben oder schneereiche Winter mit lang anhaltenden Kältewellen (siehe hierzu auch Abschn. 2.2.2 Zukünftiges Klima). Selbst die Abfolge von mehreren Jahren mit ungewöhnlich niedrigen Temperaturen in einer Jahreszeit ist möglich, da die natürlichen dekadischen Schwankungen den Klimawandel überlagern können. Der vom Menschen verursachte langfristige Klimawandel setzt sich dennoch weiter fort. Das beobachtete Klima ergibt sich aus der Überlagerung von natürlichen Schwankungen und dem anthropogenen Klimawandel. Auch wenn die dekadischen Schwankungen den Klimawandel teilweise überdecken, können sie bei einer positiven Überlagerung zu neuen Extremen und Ausprägungen der Witterung führen, die bisher noch nicht beobachtet wurden.

Generell beeinflusst der vom Menschen verursachte Landnutzungswandel, besonders im städtischen Bereich den Energie- und Stoffaustausch zwischen Boden, Wasser, Bio- und Atmosphäre (Auer et al. 1989; Zebisch et al. 2018). So werden dort die Straßen und Gebäude durch die Sonnenenergie stark erwärmt. Diese speichern die Wärmeenergie während des Tages und geben sie in der Nacht wieder ab (Žuvela-Aloise et al. 2018). Dieser Effekt erzeugt die sogenannte städtische Wärmeinsel und führt dazu, dass in windschwachen, wolkenlosen Nächten die Temperatur innerhalb der Stadt um mehrere Grad wärmer ist als im nichtverbauten Umland (Böhm 1979; Trimmel et al. 2019). Dies ist besonders während sommerlicher Hitzewellen ein Problem, da hier in Städten teilweise die Temperatur in der Nacht nicht mehr unter $20^{\circ} \mathrm{C}$ absinkt. Dadurch werden die Erholungsphasen vom Hitzestress für den Menschen immer kürzer und auch das Kühlen der Gebäude durch nächtliches Lüften weniger effizient (APCC 2018). Da der Prozess der Urbanisierung mit anhaltendem Wachstum städtischen Lebensraums in Österreich weiter voranschreitet, werden in Zukunft mehr Menschen vom Effekt der städtischen Wärmeinsel betroffen sein und dieser Effekt wird sich weiter intensivieren (APCC 2018). In Kombination mit dem Temperaturanstieg durch den Klimawandel wird dies zu einer massiven Zunahme der Hitzebelastung in unseren Städten führen und sicherlich auch den Städtetourismus beeinflussen (Trimmel et al. 2019).

\subsection{Klimawandel - speziell bezogen auf Österreich}

\subsubsection{Vergangenes Klima}

\section{Lufttemperatur und Luftdruck}

Die Aussagen über das vergangene Klima in Österreich beziehen sich auf die instrumentelle Periode der letzten 130-250 Jahre und die für den Tourismus relevanten meteorologischen Größen. Für die Zeit davor und für weitere meteorologische Größen können die Daten bei Auer et al. (2014) nachgelesen werden.

Seit der vorindustriellen Zeit ist die mittlere jährliche Lufttemperatur im Tiefland Österreichs um $1,8^{\circ} \mathrm{C}$ angestiegen (1989-2018 vs. 1850-1900), d. h. etwa doppelt so stark wie im globalen Mittel (Abb. 2.1 und ZAMG 2020b; APCC 2014) und $20 \%$ über dem Anstieg der mittleren Lufttemperatur von $1,5^{\circ} \mathrm{C}$ über den globalen Landflächen (IPCC 2019a). Ein Großteil dieser Erwärmung fand in den letzten vier Dekaden seit 1980 mit einer enormen Rate von fast $0,5^{\circ} \mathrm{C}$ pro Dekade statt. Die Hauptursache für die im Vergleich zum globalen Mittel stärkere Temperaturzunahme ist raschere Erwärmung der Luft über Landflächen gegenüber der über thermisch trägeren Ozeanen (FAQ 2.1, Figure 2 in Hartmann et al. 2013; IPCC 2019a), aber auch der Einfluss gestiegener bodennaher solarer Einstrahlung seit den 1980er-Jahren spielt höchstwahrscheinlich eine wichtige Rolle (Scherrer und Begert 2019). Ob dieser Anstieg vorwiegend auf abnehmende Aerosolekonzentrationen in der Luft (,global brightening“s. u.) oder auch auf abnehmende Bewölkung infolge des eventuell nordwärts verlagerten subtropischen Hochdruckgürtels zurückzuführen ist, wird in der Fachliteratur noch diskutiert. Letzteres würde die beobachtete gemeinsame Zunahme von Luftdruck und Sonnenschein bis ca. zum Jahr 1990 erklären (siehe Abschn. Sonnenschein und Auer et al. 2007, 2009), nicht aber die markante Trendumkehr des Luftdrucks um 1990 bei nach wie vor steigenden Werten der Sonnenscheindauer (Auer et al. 2014). Trends aus vorhandenen homogenisierten direkten Beobachtungen der Bewölkungsmenge bis 2012 liefern kein eindeutiges Indiz zu dieser Fragestellung (Auer et al. 2014). Die Erwärmungsphase des frühen 20. Jahrhunderts wird als Übergang vom natürlichen Klima, in dem für den Strahlungsantrieb solare und vulkanische Einflüsse praktisch allein ausschlaggebend waren, zum menschlich beeinflussten Klima mit einsetzendem anthropogenen Treibhauseffekt gesehen (Auer et al. 2014; Abb. 2.2). Die Abkühlung nach der Jahrhundertmitte in den Jahrzehnten des Wirtschaftswachstums ist durch den Effekt des anthropogenen Aerosolausstoßes ${ }^{2}$, hauptsächlich von Sulfat aus der Verbrennung von Kohle und Erdöl, verursacht, indem die Aerosole (Luftpartikel) die am Erdboden eintreffende Sonnenstrahlung durch Absorption

\footnotetext{
2 Aerosole sind feine Partikel in der Luft.
} 
und Reflexion abmindern (sog. Global Dimming). Als gegen Ende des 20. Jahrhunderts Maßnahmen zur Luftreinhaltung wirksam werden und sich zusätzlich die Treibhausgasemissionen aus Industrie und Verkehr verstärken, tritt die Erde endgültig ins anthropogene Treibhauszeitalter ein (sog. Global Brightening; Wild 2009; Nabat et al. 2014; Manara et al. 2016; Pfeifroth et al. 2018). Es ist extrem wahrscheinlich, dass mehr als die Hälfte des beobachteten Anstiegs der globalen Mitteltemperatur zwischen 1951 und 2010 durch den anthropogenen Anstieg der Treibhausgaskonzentration und Folgeeffekte anderer anthropogener Antriebsformen verursacht wurde (IPCC 2014).

Innerhalb Österreichs zeigen sich die Langzeitveränderungen der Temperatur in großer räumlicher Übereinstimmung. Nennenswerte Unterschiede in der Temperaturentwicklung gab es weder regional noch in Abhängigkeit von der Seehöhe: In den Gipfelregionen hat sich die Atmosphäre seit der vorindustriellen Zeit ebenfalls um 1,8 ${ }^{\circ} \mathrm{C}$ erwärmt (1989-2018 vs. 1850-1900; Auer et al. 2007; Böhm 2012; Auer et al. 2014; ZAMG 2020b). Die Temperaturentwicklung in höheren Luftschichten, abgeleitet aus homogenisierten Radiosondenmessungen, ist in $3000 \mathrm{~m}$ Höhe dem Verlauf an hochalpinen Stationen sehr ähnlich (Haimberger et al. 2012; Auer et al. 2014). Saisonal betrachtet ist die Erwärmung im Tiefland im Frühjahr und Winter am stärksten $\left(+2,1^{\circ} \mathrm{C}\right.$ bzw. $\left.+2,3{ }^{\circ} \mathrm{C}\right)$, gefolgt vom Sommer $\left(+1,8^{\circ} \mathrm{C}\right)$, am schwächsten ist sie im Herbst $\left(+1,3{ }^{\circ} \mathrm{C}\right.$; Abb. 2.2). Den einzigen signifikanten Unterschied in der Erwärmung zwischen tiefen und hohen Lagen gibt es im Winter $\left(+1,7^{\circ} \mathrm{C}\right.$ in den Gipfelregionen vs. $+2,3{ }^{\circ} \mathrm{C}$ im Tiefland). Nach einer kurzen Abkühlungsphase (zwischen 1995 und 2005 im Tiefland bzw. 1989 und 2012 in den Gipfelregionen) haben die Wintertemperaturen wieder deutlich zugenommen (Abb. 2.2; Auer et al. 2014; Olefs et al. 2019). Die hochalpinen Wintertemperaturen einzelner Stationen der Ostalpen nördlich und entlang des Alpenhauptkamms haben langfristig gesehen statistisch hochsignifikant zugenommen (z. B. $+1,9$ bis $+2,4{ }^{\circ} \mathrm{C}$ an den Stationen Zugspitze, Sonnblick, Säntis, Obergurgl, Patscherkofel, Schmittenhöhe innerhalb der letzten 90 Jahre). Gleichzeitig sind sie auch von der stärksten Jahr-zu-Jahr-Variabilität aller vier Jahreszeiten geprägt (s. Abschn. 2.1): die langfristige Erwärmung ist überlagert von kurz- bis mittelfristigen natürlichen Schwankungen des Klimas (Jahr-zu-Jahr bis zu ca. 20 Jahren; Gobiet et al. 2017). Diese führen dazu, dass die 30-jährigen Unterperioden A, B und C in Abb. 2.3 unterschiedlich starke und nicht signifikante Erwärmungstrends aufweisen und der Zeitraum 1989 bis 2012 sogar durch eine Abkühlung gekennzeichnet ist. Saffioti et al. (2016) konnten eindeutig zeigen, dass diese europäische winterliche Abkühlungsperiode ein Resultat der natürlichen Klimavariabilität war. Dies bedeutet gleichzeitig, dass für diese kürzeren Zeiträume und lokale bis regionale Betrachtungen, in denen natürliche Klimaschwankungen dominieren, zuverlässige Vorhersagen für die Zukunft derzeit noch nicht möglich sind, ganz im Gegensatz zum langfristigen Erwärmungstrend. Diese natürlichen Klimaschwankungen werden wesentlich von Wechselwirkungen zwischen Atmosphäre und Ozean verursacht. So konnte gezeigt werden, dass z. B. die sog. nordatlantische Oszillation (NAO) starken Einfluss auf die Jahr-zu-Jahr- und dekadischen Schwankungen der Wintertemperatur in Europa und auch Österreich hat (Hurrell 1995; Schöner et al. 2009).

Als kleinskaligere Phänomene sind sogenannte Temperaturinversionen z. B. wichtig für die Erzeugung von technischem Schnee im Herbst und Winter, da sie mit der Bildung bodennaher Kaltluftseen verbunden sind, welche die Rahmenbedingungen für die Kunstschneeproduktion verändern. Sie sind geprägt durch eine Umkehr des vertikalen Temperaturgradienten, d. h., die Luft ist in höheren Lagen wärmer als darunter. Aus täglichen flächigen Beobachtungsdaten konnte abgeleitet werden, dass solche Inversionen in der Periode 1961 bis 2017, insbesondere in den Monaten Oktober, Dezember und Januar, im Süden Österreichs sowie zentralalpin seltener und weniger intensiv geworden sind (Hiebl und Schöner 2018).

Der deutliche Temperaturanstieg führte auch zu einer Veränderung der temperaturabhängigen Extremwerte. So stieg z. B. die Anzahl von Sommertagen (Tageshöchstwert $\geq 25^{\circ} \mathrm{C}$ ) und Tropennächten (Tagestiefstwert nicht unter $20^{\circ} \mathrm{C}$ ) im Zeitraum 1948 bis 2010 signifikant (Nemec et al. 2013). Die Temperaturvariabilität, also die Wechselhaftigkeit zwischen kalten und warmen Extremen, hat sich hingegen seit Ende des 19. Jahrhunderts in Österreich langfristig nicht signifikant geändert (Hiebl und Hofstätter 2012).

Die Temperaturänderung seit Ende des 19. Jahrhunderts wurde auch durch die völlig unabhängig gemessene Entwicklung des Luftdrucks in unterschiedlichen Höhen bestätigt (Böhm et al. 1998; Böhm 2012).

\section{Niederschlag}

Abgesehen von der ersten Hälfte des 19. Jahrhunderts, die relativ niederschlagsreich ausfiel, und der trockensten Phase der Messgeschichte in den 1860er-Jahren (Haslinger et al. 2018), gestaltete sich das Niederschlagsklima im Mittel über ganz Österreich auch saisonal ohne ausgeprägte langfristige Schwankungen (Auer et al. 2007). Allerdings zeigen sich in einzelnen Regionen Österreichs unterschiedliche, teilweise sogar gegenläufige Niederschlagstrends, die vor allem im Winter zu beobachten sind: In Westösterreich (Vorarlberg, Nordtirol) nahm der Niederschlag, über den gesamten Zeitraum seit 1858 betrachtet, um ca. 10-15\% zu, während er im Südosten Österreichs (Unterkärnten, West- und Oststeiermark, Südburgenland) über die letzten 200 Jahre hinweg abnahm (Böhm 2006).

\section{Sonnenschein}

Bei der Entwicklung der Sonnenscheindauer ist seit Ende des 19. Jahrhunderts eine zweistufige Zunahme zu beobach- 
ten: Die erste Zuwachsphase vom Ende des 19. zur Mitte des 20. Jahrhunderts hin erreichte ihren Höhepunkt in den sonnenreichen Nachkriegssommern der späten 1940er- und frühen 1950er-Jahre. Der Rückgang des sommerlichen Schönwetters in den 1950er-, 1960er- und 1970er-Jahren schlägt sich deutlich in den Jahressummen des Sonnenscheins durch. Nach einem Wendepunkt um 1980 folgt die zweite Phase rascher Sonnenscheinzunahme (um ca. 10\%) der letzten 35 Jahre, wobei 2003 und 2011 herausragen. Die auffallende Ähnlichkeit der zweistufigen Entwicklung der Temperatur- und Sonnenscheinkurven im 20. und 21. Jahrhundert deutet auf eine Rolle der solaren Einstrahlung beim überproportionalen Temperaturanstieg im Alpenraum gegenüber den globalen Landflächen hin (siehe Abschn. Lufttemperatur; Scherrer und Begert 2019).

\section{Wind}

Überschreitet die Windgeschwindigkeit $75 \mathrm{~km} / \mathrm{h}$ oder 9 Beaufort, so nennt man diesen Wind Sturm (WMO 1970). Zur robusten Beschreibung von Änderungen des Windklimas eignen sich lange zurückreichende Luftdruckmessreihen, aus denen räumliche Luftdruckunterschiede berechnet werden, besser als direkte Windmessungen (Auer et al. 2007; Matulla et al. 2008). Eine Untersuchung von Matulla et al. (2008) zeigt in drei untersuchten Regionen Europas (Nordwest-, Nord- und Mitteleuropa) keinen langfristigen Trend zu mehr Stürmigkeit. Auch andere Untersuchungen über das Sturmklima über Nordwesteuropa (Feser et al. 2015) verdeutlichen zwar die hohe Variabilität auf der jährlichen und dekadischen (10- bis 50-jährigen) Zeitskala, zeigen aber keine Zunahme der Stürmigkeit während der letzten 100 Jahre. Die Zugbahnen der Tiefdruckgebiete über Europa haben sich demnach weiter nach Norden bzw. Nordosten hin verlagert. Die Ausbildung von kleinräumigen, thermisch induzierten Windsystemen wie Hangwinden und Thermik sind an eine ganze Reihe von gleichzeitig auftretenden meteorologischen Grundvoraussetzungen gekoppelt (Rafelsberger 2007): Neben der Topografie, dem Untergrund, der vertikalen atmosphärischen Schichtung, den Feuchte-, Temperatur- und Strahlungsverhältnissen (und somit auch der Jahreszeit) darf keine Beeinträchtigung durch Fronten oder Föhnwinde gegeben sein und somit ist auch eine Abhängigkeit von der Häufigkeit bestimmter Wetterlagen gegeben. Studien zu möglichen langfristigen Veränderungen in der Vergangenheit sind für Österreich derzeit keine bekannt. Aussagen über kleinräumige in Zusammenhang mit Gewittern entstehende Windböen sind an Konvektion gebunden, über welche derzeit für die Vergangenheit in Österreich keine verlässlichen Aussagen hinsichtlich Trends gemacht werden können (siehe nächster Abschnitt). Bei der Betrachtung von ganz Europa gibt es aber mittlerweile deutliche Anzeichen für einen Anstieg dieser lokalen Windböen (Rädler et al. 2018).

\section{Starkniederschläge, Gewitter und Hagel}

Starkniederschläge definieren sich aus einer Kombination von Intensität und Dauer des Niederschlags. Für flächige Starkniederschlagsereignisse, die durch Tiefdruckgebiete oder Staueffekte verursacht werden und das ganze Jahr auftreten können, lassen sich derzeit für Österreich keine einheitlichen und kaum signifikante Trends in der Vergangenheit erkennen (Hofstätter et al. 2018; Pistotnik et al. 2020). Kleinräumige Starkniederschläge sind an Konvektion gebunden und treten vorwiegend im Sommerhalbjahr auf. Aufgrund der zu geringen räumlichen Dichte konventioneller meteorologischer Messnetze und der zu geringen zeitlichen Auflösung der Messungen vor Beginn der Automatisierung Ende der 1980er-Jahre können derzeit keine verlässlichen Aussagen über vergangene Trends konvektiver Starkniederschläge respektive Gewitter gemacht werden (Pistotnik et al. 2020). Eine Analyse des flächigen Niederschlagsbeobachtungsdatensatzes Spartacus (Hiebl und Frei 2018) deutet im Mittel über Österreich für das Gesamtjahr auf eine Abnahme der Häufigkeit von schwachen oder moderaten Niederschlagstagen und eine Zunahme von starken bis extremen Niederschlagstagen hin, insbesondere im Sommer und Herbst (Chimani et al. 2016). Die Gesamtanzahl der Niederschlagstage bleibt im Jahresmittel dabei gleich, es kommt somit nur zu einer Verschiebung in den Intensitäten.

Aus physikalischen Gründen - eine wärmere Atmosphäre kann mehr Wasserdampf aufnehmen - muss man von einer Zunahme der Niederschlagsintensität von ca. $7 \%$ pro ${ }^{\circ} \mathrm{C}$ ausgehen (Clausius-Clapeyron-Gesetz). Auswertungen von Stundenniederschlägen der Station Wien Hohe Warte (Formayer und Fritz 2017) ergeben eine Zunahme der Niederschlagsintensität von etwa $10 \%$ pro ${ }^{\circ} \mathrm{C}$ Temperaturanstieg, Schroeer und Kirchengast (2018) finden Werte von bis zu $14 \%$ pro ${ }^{\circ} \mathrm{C}$ basierend auf 10 -Minuten-Messungen an extremen Niederschlagstagen (98. Perzentil) in Südostösterreich. Beide Werte überschreiten deutlich den durch das theoretische Clausius-Clapeyron-Gesetz vorgegebenen Grenzwert, was durch zahlreiche weltweite Studien für kurzzeitige, konvektive Niederschlagsereignisse bestätigt wird (z. B. Ivancic und Shaw 2016; Lochbihler et al. 2019). Als Hauptgrund für die höhere Zunahme wird vermehrt frei werdende Kondensationswärme durch den höheren Feuchtegehalt genannt, was die Labilität der Luftschichtung erhöht und somit die Konvektion und Niederschlagsbildung zusätzlich verstärkt (Lenderink und van Meijgaard 2008, 2010). Nachdem die Ausbildung von Hagel an Konvektion gebunden ist und somit auch ein sehr kleinräumiges Phänomen darstellt, für das es zudem keine systematische meteorologische Messung gibt, sind auch hier verlässliche Aussagen über klimawandelbedingte vergangene Veränderungen nicht möglich.

Europaweit betrachtet wird verlässlich davon ausgegangen, dass Starkniederschläge in den letzten Jahrzehnten in vielen Teilen Europas zugenommen haben, wenn auch mit 

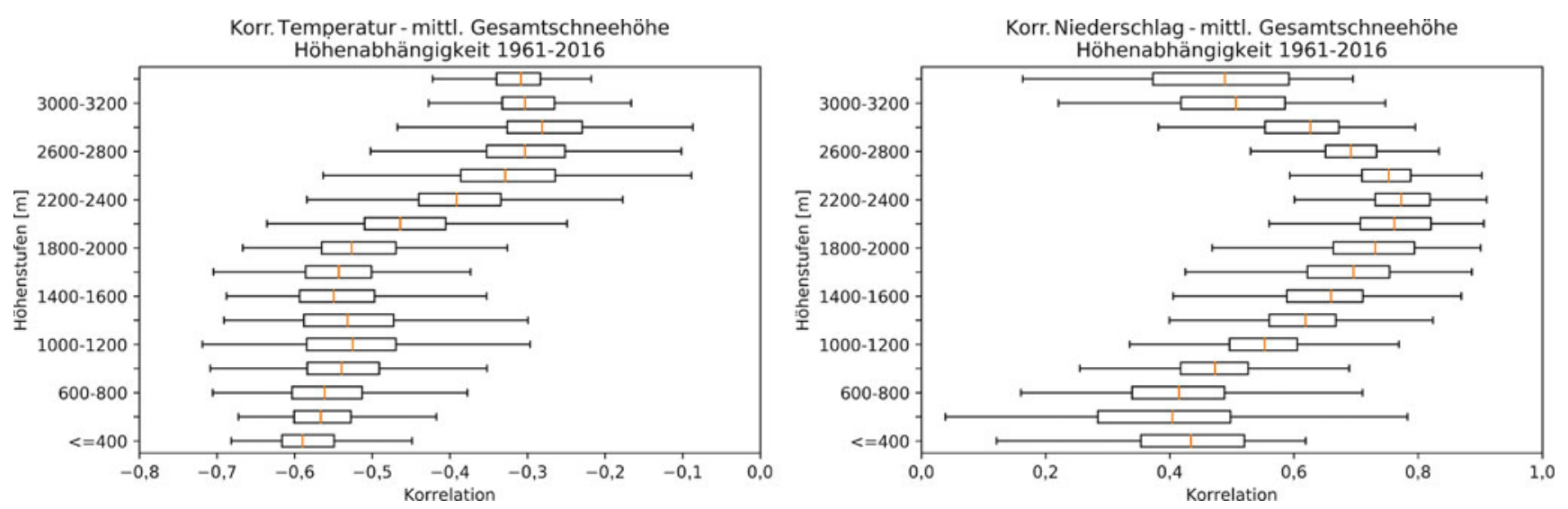

Abb. 2.4 Pearson-Korrelationskoeffizient zwischen mittlerer Gesamtschneehöhe (Nov. bis Apr.) und Temperatur (links) bzw. Niederschlagssumme (rechts) in Österreich für den Zeitraum 1961/1962 bis 2016/2017 nach Höhenstufen. (Olefs et al. 2019)

regionalen Abweichungen (Hartmann et al. 2013; Lehmann et al. 2015; Fischer und Knutti 2016). Ergebnisse eines mit Ereignisdaten validierten statistischen Modells, das für Europa mit flächigen Beobachtungsdaten im Zeitraum 1979 bis 2016 angetrieben wurde (ERA-Interim), zeigen eine deutliche Zunahme der Auftrittswahrscheinlichkeit von Blitz-, Windund großen Hagelereignissen. Die Zunahme wird dabei auf eine Labilisierung der Luftschichtung durch vermehrt frei werdende Kondensationswärme aufgrund eines höheren Feuchtegehalts zurückgeführt (Rädler et al. 2018).

\section{Saisonale Schneedecke}

Die Schneedeckenentwicklung im Laufe der Saison ist durch den räumlich sehr variablen Schneedeckenauf-/-abbau gekennzeichnet. Beim Schneedeckenaufbau spielt neben der Schneefallgrenze insbesondere die Niederschlagsmenge eine große Rolle. Der Schneedeckenabbau ist andererseits gesteuert über die sogenannte Energiebilanz der Schneeoberfläche, d. h., wie viel Energie dem Schnee netto zur Schmelze oder zum Übergang in den gasförmigen Zustand (Sublimation) zur Verfügung steht (Pomeroy und Brun 2001). Angenähert wird die Energiebilanz häufig über die deutlich einfacher messbare Lufttemperatur, was insbesondere für horizontale Flächen und bis in mittlere Höhenlagen eine ausreichend genaue Annäherung an die tatsächliche Energiebilanz darstellt (Hock 2003). Aufgrund der starken Abhängigkeit des Schnees von Lufttemperatur und Niederschlag sind räumliche Unterschiede in der zeitlichen Schneedeckenentwicklung vor allem bedingt durch die Seehöhe, schneebringende Luftmassen und deren Herkunft (Formayer und Haas 2011; Schöner et al. 2018) und regional- bis lokalklimatologische Gegebenheiten (z. B. Föhnhäufigkeit, Inversionen, Stauniederschläge, Absinkeffekte der Schneefallgrenze) festzustellen. Abb. 2.4 zeigt höhenabhängige Korrelationen der Schneehöhe mit der Lufttemperatur bzw. dem Niederschlag auf Basis von flächigen Beobachtungsdatensätzen im Zeitraum 1961-2016 für ganz
Österreich und verdeutlicht, dass der Einfluss der Temperatur auf die mittlere Schneehöhe bis in Höhen von etwa 2000 m praktisch konstant bleibt und darüber deutlich abnimmt. Andererseits ist der Einfluss des Niederschlags bereits ab ca. $1300 \mathrm{~m}$ größer als der der Temperatur. Für die Vergangenheit lässt sich also sagen: Schnee in Höhenlagen unter etwa $2000 \mathrm{~m}$ reagiert relativ empfindlich auf höhere Temperaturen, über etwa 1300 m kann dieser Effekt aber durch zusätzlichen Niederschlag (falls vorhanden) kompensiert werden. Oberhalb von $2000 \mathrm{~m}$ spielt die Temperatur eine untergeordnete Rolle (Scherrer et al. 2004; Schöner et al. 2018; Gobiet et al. 2018; Olefs et al. 2019), hier ist die Schneehöhe aufgrund ohnehin niedriger Temperaturen in erster Linie vom Niederschlag abhängig.

Die winterliche Schneedecke weist von Jahr zu Jahr und multidekadisch (d. h. über mehrere Jahrzehnte hinweg) große natürliche Schwankungen auf und reagiert innerhalb verschiedener Höhenlagen und Regionen unterschiedlich auf Temperatur- und Niederschlagsänderung und somit auf Klimaänderungen (Roth 2018). Die hohe zeitliche Variabilität überdeckt das langjährige Klimasignal und erschwert Aussagen über Trends im Bereich der Schneedecke (Schöner et al. 2018). Wechselwirkungen zwischen der Atmosphäre und den Ozeanen werden als Hauptgrund für die natürliche kurz- bis mittelfristige Klimavariabilität der Schneedecke gesehen (Scherrer et al. 2004). Die nordatlantische Oszillation erklärt z. B. großteils die Jahr-zu-Jahr-Schwankungen der Schneedecke in den südlichen Regionen der Schweiz und die dekadische Variabilität in südlichen und nördlichen Regionen der Schweiz (Scherrer et al. 2004) während die atlantische multidekadische Oszillation (AMO) eindeutige Zusammenhänge mit der Reduktion der alpinen Schneefälle im Frühjahr aufweist (Zampieri et al. 2013). Um robuste Schlussfolgerungen bzgl. der Schneedeckenreaktion auf Klimaänderungen sowie der räumlichen und zeitlichen Variabilität der Schneebedingungen ziehen zu können, werden somit langjährige und kon- 

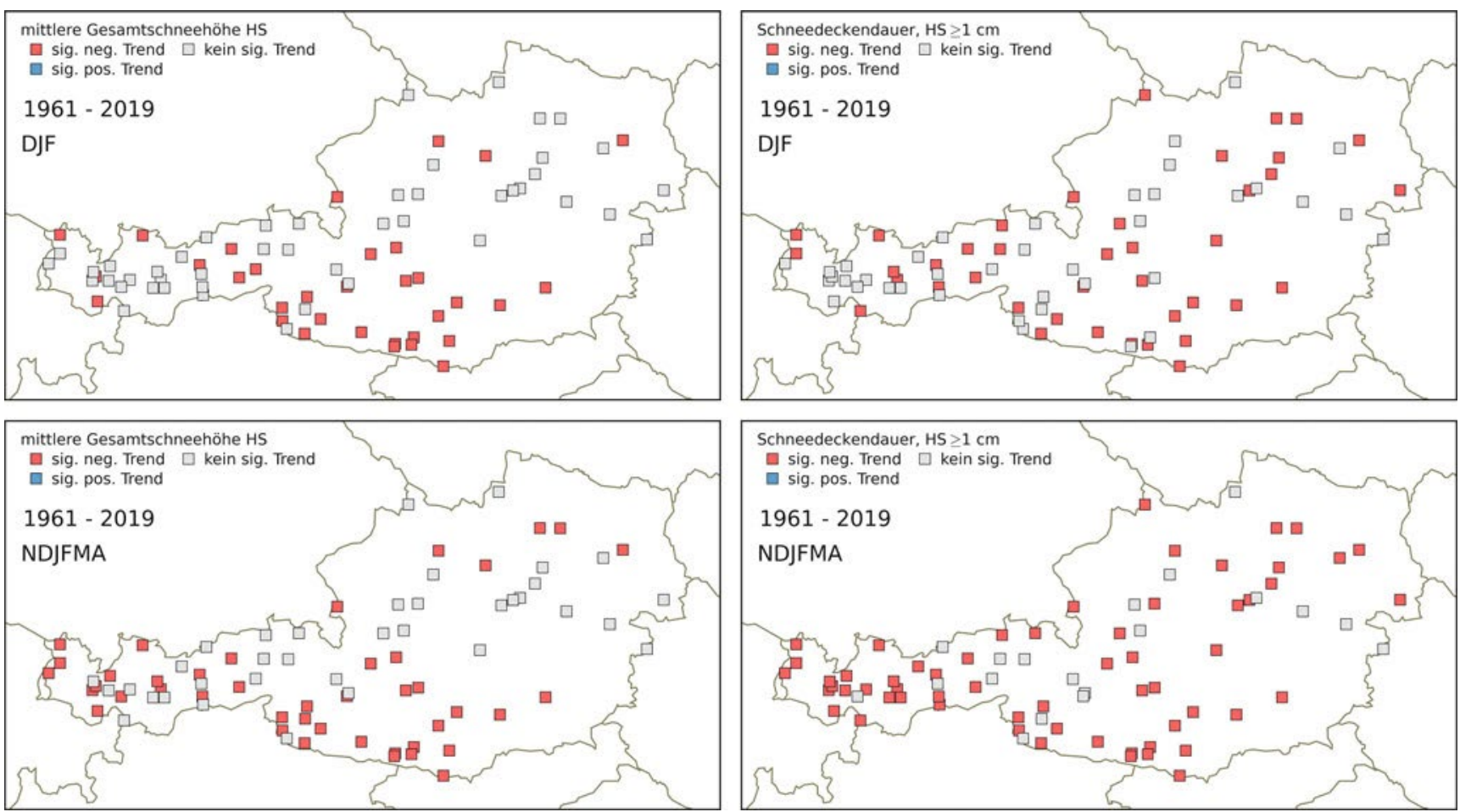

Abb. 2.5 Trendanalyse nach Mann-Kendall (Signifikanzniveau =0,05) der saisonalen mittleren Gesamtschneehöhe (links) und der saisonalen Schneedeckendauer (rechts) für die Wintersaisonen Dezember bis Februar (oben) und November bis April (unten). Der Test umfasst die Wintersaisonen von 1961 bis 2019. Rot (Blau) entspricht signifikant negativ (positiv) bezüglich eines $95 \%$-Konfidenzintervalls und Grau kennzeichnet keinen signifikanten Trend (Olefs et al. 2019). Es wurden keinerlei signifikant positive Trends (blau) errechnet

sistente Zeitreihen benötigt. Die bisher umfassendste Arbeit zur Homogenisierung und Analyse von Langzeitmessreihen der Gesamtschneehöhe und Neuschneemenge in Österreich wurde dabei im Rahmen des Projekts SNOWPAT gemacht (Schöner et al. 2018). Die Auswertungen für den Zeitraum 1961-2012 zeigen langfristig signifikant abnehmende Schneehöhen und Schneedeckendauern, insbesondere im Süden, aber in abgeschwächter Form auch im Westen Österreichs. Im Nordosten hingegen wurden keine signifikanten Trends gefunden. Diese generellen Muster werden sowohl mit Daten bis 2019 (Abb. 2.5; Olefs et al. 2019) als auch durch zahlreiche Studien aus der Schweiz (z. B. Beniston 1997; Laternser und Schneebeli 2003; Scherrer et al. 2004; Marty 2008; Serquet et al. 2011; Klein et al. 2016; Marty et al. 2017a) und dem Alpenraum (Beniston et al. 2018) bestätigt. Zwei unabhängige numerische Experimente mit räumlich verteilten Schneedeckenmodellen über einen vergleichbaren Zeitraum kommen zu einem ähnlichen Ergebnis (Olefs et al. 2017; Marke et al. 2018). Die relativen Abnahmen erstrecken sich über alle untersuchten Höhenlagen bis $2100 \mathrm{~m}$ Seehöhe und betragen 30-90 \% (bzw. 60 \% oberhalb $1000 \mathrm{~m}$ Seehöhe), bei der Schneedeckendauer liegt dieser Wert bei 0-70 \% (bzw. $25 \%$ > 1000 m Seehöhe; Abb. 2.6). In absoluten Zahlen hat die Schneedeckendauer im österreichischen Flächenmittel von 1961 bis 2016 um -44 Tage hochsignifikant abgenommen (Olefs et al. 2017; entspricht einer Rate von -8 Tagen pro Dekade und liegt somit etwas oberhalb des globalen Mittels von -5 Tagen pro Dekade; IPCC 2019b). Die Trendstärke ist insbesondere bei der Schneedeckendauer stark von der Seehöhe abhängig. Abnahmen größer $80 \%$ werden nur an Stationen südlich des Alpenhauptkamms beobachtet. Hier spiegelt sich neben der langfristigen Erwärmung auch eine Reduktion in der Intensität und/oder Häufigkeit von Südwestwetterlagen im Zeitraum von ca. Ende der 1980er-Jahre bis 2003 wieder (Olefs et al. 2019). Aigner et al. (2018) finden für 122-, 100- und 30-jährige Zeitfenster vor heute keine statistisch signifikanten Trends bei der jährlichen maximalen Schneehöhe und der Schneedeckendauer. Die Trendanalysen wurden allerdings auf Basis von Zeitreihen erstellt, die nicht homogenisiert wurden und zudem aus mehreren Stationen in klimatisch völlig unterschiedlichen Regionen und unterschiedlicher Seehöhe gemittelt wurden. Aus diesen Gründen und aufgrund mangelnder methodischer Transparenz ist diese Studie als nicht wissenschaftlich einzustufen und die Ergebnisse sind nicht belastbar.

Zur Aufrechterhaltung des Skibetriebs im Winter spielt die technische Schneeproduktion eine wesentliche Rolle (Steiger und Abegg 2013). Die atmosphärischen Rahmenbedingungen dafür sind durch die Feuchttemperatur vorgegeben, die sowohl Information über die Temperatur als auch den Feuchtegehalt der Luft beinhaltet. Die wenigen existierenden Studien für Österreich zeigen eine generelle langfristige Abnahme 


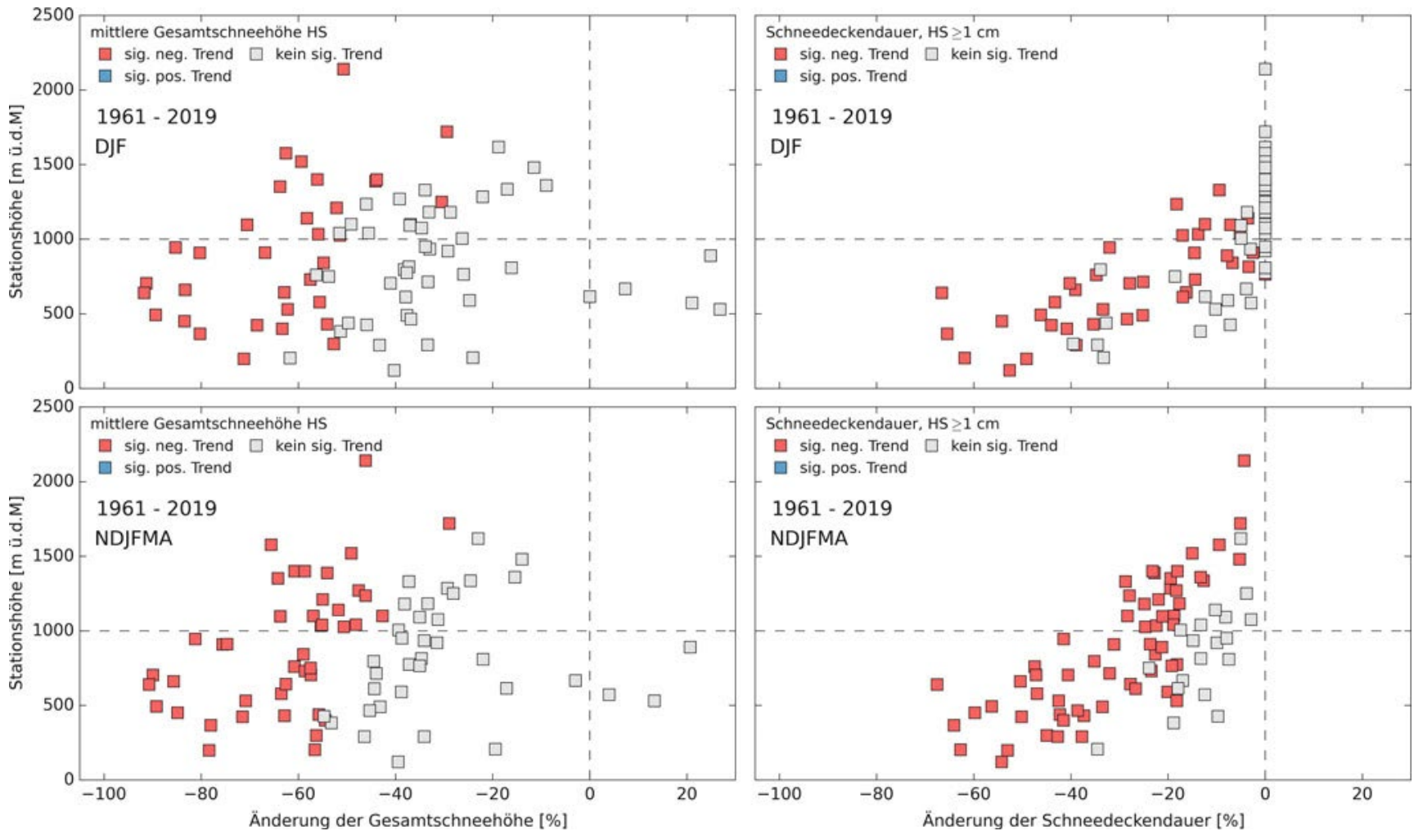

Abb. 2.6 Prozentuelle Änderung (abgeleitet aus den linearen Trends (Theil-Sen Slope)) im Zeitraum 1961 bis 2019 in Abhängigkeit der Seehöhe der saisonalen mittleren Gesamtschneehöhe (links) und der saisonalen Schneedeckendauer (rechts) für die Wintersaisonen Dezember bis Februar (oben) und November bis April (unten). Die Trendsignifikanz bezüglich eines 95\%-Konfidenzintervalls wurde nach Mann-Kendall abgeschätzt. Rot (Blau) entspricht signifikant negativ (positiv) und Grau kennzeichnet keinen signifikanten Trend (Olefs et al. 2019). Es wurden keinerlei signifikant positive Trends (blau) errechnet

der potenziellen Beschneitage pro Wintersaison (Tage mit Feuchttemperatur $<-2{ }^{\circ} \mathrm{C}$ ). Innerhalb des Früh- und Kernwinters fallen die deutlichsten Änderungen auf den Monat Dezember, aufgrund des Einflusses der Luftfeuchte und weiterer mikroklimatischer Prozesse (z. B. Inversionen) sind die signifikanten Trends aber insbesondere auf monatlicher Basis nicht immer über einheitliche Perioden verteilt (Olefs et al. 2010; Hartl et al. 2018). Die Studie von Spandre et al. (2015) findet ähnliche Muster für Frankreich. Für eine Analyse allgemeinerer Auswirkungen des Klimawandels auf den Skibetrieb sei an dieser Stelle auch auf Abschn. 6.3.1 verwiesen. Andere technische Maßnahmen zur Erhöhung der Schneesicherheit (Abdecken, Wasserinjektion, Kompaktion) wurden in früheren Experimenten in Gletscherskigebieten miteinander verglichen und ausgiebig wissenschaftlich evaluiert. Ergebnisse haben gezeigt, dass Abdecken mit Geotextilien ${ }^{3}$ oberhalb der Waldgrenze am effizientesten ist und die beobachtete natürliche Abschmelzung um ca. $60 \%$ reduzieren

\footnotetext{
${ }^{3}$ Flächige, wasserdurchlässige Textilien (in diesem Fall hergestellt aus künstlichen Stoffen wie Polypropylen oder Polyethylen), die herkömmlich als Baustoff und zu geotechnischen Sicherungsarbeiten verwendet werden.
}

kann (Olefs und Fischer 2008; Olefs und Lehning 2010). Vergleichbare Experimente mit natürlicher Abdeckung mittels Sägespäne in mittleren Höhenlagen der Schweiz über den Sommer haben sehr ähnliche Ergebnisse gebracht (Grünewald et al. 2018).

\section{Gletscher und Permafrost}

Die derzeit 890 österreichischen Gletscher bedeckten im Jahr 2006 (aktuelles österr. Gletscherinventar) eine Fläche von $414,1 \mathrm{~km}^{2}$ mit einem Eisvolumen von $15,9 \mathrm{~km}^{3}$ (Helfricht et al. 2019). Seit dem letzten Maximalstand der Alpengletscher im Jahr 1850 (letzter Höhepunkt der sogenannten kleinen Eiszeit) haben sie damit um $56 \%$ an Fläche verloren (Fischer et al. 2015). Diese Abnahmen folgen somit dem globalen Trend (IPCC 2019b).

$47 \%$ dieser Gletscher sind heute kleiner als 10 Hektar $\left(0,1 \mathrm{~km}^{2}\right)$. Im Zeitraum 2006-2016 gab es noch einen geschätzten weiteren Volumenverlust von ca. $3,5 \mathrm{~km}^{3}$ Eis (Helfricht et al. 2019). Für Gletscherskigebiete bedeutet der Gletscherschwund einen erhöhten, laufenden technischen Anpassungsaufwand (z. B. baulichen Schutz der am Gletscher errichteten Infrastruktur, wie z. B. Liftstützen; Fischer et al. 2011). Effektive Maßnahmen zur künstlichen Reduktion der 
Massenverluste durch z. B. Abdeckvliese wurden ausgiebig getestet, wissenschaftlich bewertet (Olefs und Obleitner 2007; Olefs und Fischer 2008; Olefs und Lehning 2010) und werden seitdem angewendet. Aufgrund des hohen Materialund Personalaufwandes bleiben diese Eingriffe jedoch auf neuralgische Stellen der Skigebiete beschränkt. Nachweisbar ist auch ein langfristig positiver Effekt auf die lokale Massenbilanz dieser Gletscher zehn Jahre nach Beginn der ersten Maßnahmen (Fischer et al. 2016).

Boden, der im Untergrund über mindestens zwei Jahre hinweg gefroren bleibt, wird als Permafrost bezeichnet. Dabei wird zwischen eisarmem (kontrolliert durch thermische Prozesse) und eisreichem Permafrost (kontrolliert durch Massenablagerungen, z. B. Blockgletscher) unterschieden. Das Auftreten von eisarmem Permafrost zeigt eine starke Korrelation mit der Seehöhe (als Proxy für die Jahresmitteltemperatur) und Sonneneinstrahlung, während eisreicher Permafrost an das Vorhandensein entsprechender gravitativer Massenablagerungen gebunden ist (Kenner et al. 2019). Die Messung von Permafrost erfolgt mittels Temperatursensoren in unterschiedlich tiefen, vertikalen Bohrlöchern. Die längste europäische Messreihe startete erst 1987 (Haeberli und Beniston 1998), deutlich später als bei allen anderen hier untersuchten Größen. Diese und andere Reihen zeigen einen klaren langfristigen Erwärmungstrend und eine Vergrößerung der oberflächennahen Auftauschicht (Noetzli et al. 2016), dies zeigt sich auch klar auf globaler Skala (Biskaborn et al. 2019). In Österreich beginnen derartige Messungen erst im Jahr 2006 im Sonnblickgebiet, was für Trendanalysen noch zu kurz ist (Schöner et al. 2012).

Sowohl Gletscher- als auch Permafrostrückgang haben diverse negative Auswirkungen auf die alpine Infrastruktur (z. B. hoch gelegene Skigebiete oder Verkehrswege) und den Alpinismus (s. Abschn. 7.3.2) ${ }^{4}$.

\section{Temperaturen von Oberflächen- und Fließgewässern}

Matulla et al. (2018a) zeigen, dass die Temperaturen österreichischer Seen seit Beginn der 1980er-Jahre im Sommer um ca. $2{ }^{\circ} \mathrm{C}$ gestiegen sind, im Herbst ist die Erwärmung in etwa halb so stark. Die Studie basiert auf Beobachtungsdaten von Wassertemperaturen zwölf österreichischer Seen seit 1950. Die Daten wurden homogenisiert und bis ins Jahr 1880 rekonstruiert. Auch die Temperaturen der österreichischen Fließgewässer sind seit Beginn des 20. Jahrhunderts signifikant gestiegen (Blöschl et al. 2011), wie u. a. an mehreren Messstellen der Donau nachgewiesen wurde (Dokulil und Donabaum 2014; BMLRT 2019). Daraus ergeben sich Auswirkungen auf die Fischfauna und entsprechenden Tourismus (s. Abschn. 7.3.2).

\footnotetext{
${ }^{4}$ Eine alpenweite Karte mit der derzeitigen Auftrittswahrscheinlichkeit von Permafrost ist verfügbar (Boeckli et al. 2012; https://www.geo.uzh ch/microsite/cryodata/PF_map_explanation.html), eine methodisch aktualisierte Karte ist derzeit leider nur für die Schweiz vorhanden (Kenner et al. 2019 bzw. https://map.geo.admin.ch).
}

\section{Klimatische Wasserbilanz}

Die klimatische Wasserbilanz ergibt sich aus der Differenz des Niederschlags und der potenziellen Evapotranspiration (Verdunstung) und hat direkte Auswirkungen auf die Bodenfeuchte. Sie spielt daher insbesondere für die Landwirtschaft eine wichtige Rolle. Aufgrund der aktuellen klimatischen Verteilung von Niederschlag und Verdunstung sind in Österreich vor allem Gebiete der pannonischen Tiefebene im Norden und Osten des Landes im Sommerhalbjahr von trockeneren Verhältnissen stärker betroffen als der Rest des Landes (geringe Niederschläge, hohe Verdunstung; Reniu 2017). Neben den Wetterlagen und der Verdunstung sind auch die Vorbedingungen, also die Bodenfeuchte im Frühjahr für die Ausbildung einer evtl. Dürre später im Jahr verantwortlich (Haslinger et al. 2019). Langfristig hat sich die klimatische Wasserbilanz in der Vergangenheit vor allem in den letzten Dekaden und in tiefen Lagen der warmen Jahreszeit aufgrund der temperaturund strahlungsbedingt gestiegenen Verdunstung und dadurch abnehmenden Bodenfeuchte in Richtung trockenerer Verhältnisse verschoben (Haslinger und Bartsch 2016; Trnka et al. 2016; Haslinger et al. 2019). Probleme ergeben sich für den Golftourismus und Wassersport (s. Abschn. 7.3.2).

\section{Abfluss}

In den letzten 30 Jahren haben in etwa $20 \%$ der Einzugsgebiete in Österreich die Hochwässer zugenommen (im Winter deutlich stärker als im Sommer), besonders in kleinen Gebieten nördlich des Alpenhauptkammes. Die Häufung der Hochwässer in den letzten Jahrzehnten liegt dabei im Rahmen der natürlichen Variabilität, aber auch ein Einfluss einer Klimaänderung ist nicht auszuschließen (Blöschl et al. 2011, 2017; BMLFUW 2017). In den alpinen Gebieten Österreichs treten die Niederwässer im Winter zufolge Schnee bzw. Gefrierprozesse auf, im Flachland des Ostens im Sommer zufolge Verdunstung. In den meisten Pegeleinzugsgebieten gibt es in den letzten 30 Jahren keinen signifikanten Trend der Niedrigwasserabflüsse, über $900 \mathrm{~m}$ Seehöhe in $14 \%$ bzw. $3 \%$ der Gebiete eine Zu- bzw. Abnahme, unterhalb $900 \mathrm{~m}$ Seehöhe in $10 \%$ bzw. $5 \%$ der Einzugsgebiete eine Ab- bzw. Zunahme.

\section{Sonstige touristisch relevante Klimafolgen}

Grundsätzlich beeinflusst die Klimavariabilität die Wasserqualität durch die Temperatur sowie das Wasserdargebot. In der Vergangenheit erfolgten die anthropogenen Einflüsse auf die Qualität von Wasserkörpern (Verschmutzung und Sanierung) wesentlich rascher, als sich klimatische Rahmenbedingungen verändert haben (Blöschl et al. 2011).

\subsubsection{Zukünftiges Klima}

Um der Gesellschaft informierte Entscheidungen zu ermöglichen, verlangt das aus der Umweltethik stammende 
Sicherheitsprinzip, dass die volle Bandbreite möglicher zukünftiger Auswirkungen der globalen Erderwärmung darzustellen ist (Kromp-Kolb et al. 2014). Zur Analyse dieser werden in der Klimaforschung globale und regionale Klimamodelle sowie diverse statistische Verfahren eingesetzt. Um der Unsicherheit Rechnung zu tragen, die durch das zukünftige menschliche Handeln entsteht, wurden verschiedene Szenarien entwickelt. Diese Szenarien oder Entwicklungspfade (Representative Concentration Pathways, RCPs; s. Abschn. 2.1) beschreiben unterschiedliche zukünftige Entwicklungen der Bevölkerung, der Weltwirtschaft, des technologischen Fortschritts und des menschlichen Handelns und die damit einhergehende Entwicklung klimarelevanter Treibhausgase und Aerosole und dienen als Randbedingung für die Klimamodelle. Man spricht daher auch von Klimaprojektionen (mögliche zukünftige Klimazustände) und nicht von Klimavorhersagen. Um weitere Unsicherheiten der Klimamodelle zu berücksichtigen, wie z. B. die Modellierungsmethoden an sich oder natürliche Klimaschwankungen, werden für jedes Klimaszenario viele Klimasimulationen mehrerer unterschiedlicher Modelle mit leicht unterschiedlichen Anfangsbedingungen gerechnet („Modellensemble“). Für jedes Klimaszenario ergibt sich somit eine Bandbreite an möglichen zukünftigen Entwicklungen. Im Folgenden werden die RCPs 2.6 (,Paris-Ziel“), 4.5 („wirksame Klimaschutzmaßnahmen“) und 8.5 („,business as usual") betrachtet (s. Abschn. 2.1 für eine detaillierte Beschreibung der Szenarien). Um die Auswirkungen der Emissionsszenarien global zu beschreiben, werden sogenannte globale Klimamodelle (GCMs) mit einer derzeitigen Auflösung von ca. 100-200 km verwendet. Aufgrund der groben horizontalen Auflösung von GCMs werden regionale Klimamodelle (RCMs) mit einer höheren räumlichen Auflösung in das GCM eingebettet und davon angetrieben (,nesting“ oder „dynamic downscaling“).

Für die Einschätzung der zukünftigen Klimaänderung in Österreich stehen die ÖKS15-Klimaszenarien (Chimani et al. 2016) für die Parameter Lufttemperatur (tägliches Maximum und Minimum), Niederschlag (tägliche Summe) und Globalstrahlung (Tagessummen) für den Zeitraum 1971 bis 2100 zur Verfügung. Dabei stellt der Zeitraum 1971 bis 2005 den historischen (auf Basis beobachteter Emissionen) und der Zeitraum 2006 bis 2100 die Klimaprojektionen (auf Basis von Emissionsszenarien) dar. Der ÖKS15-Datensatz besteht aus gegitterten Klimadatensätzen und Emissionsszenarien für die österreichische Klimaforschung. Das verwendete Ensemble beinhaltet 13 EURO-CORDEX (Jacob et al. 2014) RCMs (Ensemblemitglieder) und zwei unterschiedliche Emissionsszenarien (RCP 4.5 und RCP 8.5). Um der topografischen Komplexität Österreichs bestmöglich gerecht zu werden, wurden die Daten von einem ursprünglich weitmaschigen mit 12,5 km horizontaler Auflösung auf ein engmaschiges Netz von $1 \mathrm{~km}$ gebracht. Dafür wurden die Daten in einem ersten Schritt auf das Gitter von Beobachtungsdatensätzen interpoliert und anschließend pro RCM und Gitterpunkt um systematische Fehler (eng. Biases) bereinigt. Als Methode der Biaskorrektur wird das sogenannte Scaled Distribution Mapping verwendet, bei dem die Klimaänderungssignale der Modelle erhalten bleiben (Chimani et al. 2016; Switanek et al. 2017). Dadurch wird z. B. der Einfluss von Inversionswetterlagen (,im Tal ist es kälter als am Berg") oder vom regionalen Klimamodell falsch berechneter Niederschläge regional systematisch korrigiert. Wichtig zu verstehen ist, dass diese Korrektur dort am besten funktioniert, wo auch Stationsmessungen aus dem flächigen Beobachtungsdatensatz vorhanden sind. Im Jahr 2019 wurde der Datensatz um das Szenario RCP 2.6 und eine detaillierte Richtlinie inklusive Evaluierung des ÖKS15-Datensatzes erweitert (Chimani et al. 2019). Die Richtlinie richtet sich an Nutzerinnen und Nutzer des Datensatzes und klärt über Potenziale und Einschränkungen auf. Durch die Verwendung eines Ensembles aus mehreren Modellen wird eine große Bandbreite an möglichen Entwicklungen abgedeckt. Um eine Klimaänderung aus einem Ensemble abzuschätzen, wird im Folgenden der Ensemblemedian verwendet. Er entspricht dem zentralen (,durchschnittlichen“) Modell der Verteilung: Die eine Hälfte der Modelle liegt oberhalb des Medians, die andere unterhalb. Zusätzlich wird auch die Bandbreite des Ensembles angegeben, dazu werden das $5 \%$-Perzentil und das $95 \%$-Perzentil verwendet. Das Klimaänderungssignal wird in ÖKS15 immer im Vergleich zum Mittel der Klimanormalperiode 1971 bis 2000 angegeben. Zur Bewertung der Aussagekraft der Klimaänderungen des Ensembles wird einerseits der Grad der Übereinstimmung zwischen den unterschiedlichen Modellen herangezogen, andererseits wird überprüft, ob die jeweiligen Ensemblemitglieder modellintern eine signifikante Klimaänderung ergeben. Wenn viele der Modelle, die auf denselben physikalischen Grundannahmen, aber unterschiedlichen Vereinfachungen und Parametrisierungen beruhen, in ihrer Aussage übereinstimmen, kann diesem Ensemble ein größeres Vertrauen entgegengebracht werden als einem Ensemble, bei dem die Modelle zwar statistisch signifikante Änderungen anzeigen, sich aber widersprechen (Chimani et al. 2016). Aufgrund der Ungewissheit über das zukünftige menschliche Verhalten, der Komplexität des Klimasystems sowie der Unvollkommenheit der Modelle kann nicht ausgeschlossen werden, dass die tatsächliche zukünftige Klimaentwicklung aber auch außerhalb der angegebenen Bandbreiten liegen wird.

Andere Untersuchungen mit Klimamodellen für Europa kommen grundlegend zu sehr ähnlichen Ergebnissen (z. B. Einzelmodelle mit vielen Ensemble-Members, vgl. Leduc et al. 2019), die aktuellen Schweizer Klimaszenarien CH2018 basieren auf den gleichen EURO-CORDEX RCMs, verwenden allerdings eine andere Downscaling-Methodik (NCCS 2018). 


\section{Lufttemperatur}

Für die nahe Zukunft (2021-2050) zeigen die ÖKS15-Daten praktisch unabhängig vom Szenario einen weiteren Temperaturanstieg von etwa $1,3{ }^{\circ} \mathrm{C}$ (Ensemblemedian) im Vergleich zur Referenzperiode 1971-2000 (Abb. 2.7). Für die ferne Zukunft (2071-2100) zeigen die Modelle eine Erwärmung (Ensemblemedian) von $1,0{ }^{\circ} \mathrm{C}(\mathrm{RCP} 2.6), 2,1^{\circ} \mathrm{C}(\mathrm{RCP} 4.5)$ bzw. $4,0^{\circ} \mathrm{C}$ (RCP 8.5). Die Bandbreite von RCP 8.5 liegt dabei zwischen $+3,6$ und $+5,8^{\circ} \mathrm{C}$. Saisonal liegt die mögliche Erwärmung für Österreich (Flächenmittel des Ensemblemedians) in der nahen Zukunft bei $1,0^{\circ} \mathrm{C}$ (Frühling) bis $1,5{ }^{\circ} \mathrm{C}$ (Sommer/Herbst), in der fernen Zukunft bei $3,5^{\circ} \mathrm{C}$ (Frühling) bis $4,4{ }^{\circ} \mathrm{C}$ (Winter; Abb. 2.8). Mit dem Anstieg der mittleren Lufttemperatur ändern sich auch die Temperaturextrema in allen Höhenlagen. Signifikante Änderungen der Hitzetage (Tage mit Höchstwerten über $30^{\circ} \mathrm{C}$ ) ergeben sich jedoch nur für Lagen unterhalb von etwa $1000 \mathrm{~m}$. Stärkste Zunahmen sind im Sommer, im Alpenvorland, dem Flachund Hügelland und dem Klagenfurter Becken zu finden. Des Weiteren kommt es zu einem zunehmenden Auftreten von Sommer- und Hitzetagen in den Übergangsjahreszeiten. So steigt beispielsweise die Anzahl der Hitzetage in der nahen bzw. fernen Zukunft um 3 Tage (RCP 2.6; gleich für nahe und ferne Zukunft), 4 bzw. 7 Tage (RCP 4.5) und 12 bzw. 19 Tage (RCP 8.5) im Österreichmittel des jeweiligen Ensemblemedians. Massive Änderungen ergeben sich in RCP 8.5 in der fernen Zukunft mit einer Zunahme an Sommertagen (Tage mit Höchstwerten über $25^{\circ} \mathrm{C}$ ) von 50 Tagen in der Südoststeiermark und im Nordwesten Vorarlbergs sowie mit einer Zunahme von knapp über 40 Hitzetagen in der Südoststeiermark und im Tiroler Inntal. Die Anzahl der Tropennächte (Tiefstwert nicht unter $20^{\circ} \mathrm{C}$ ) steigt im Mittel um 1 Tag (nahe Zukunft) bzw. 1 bis 8 Tage (RCP 2.6/8.5) in der fernen Zukunft (Chimani et al. 2016). Am Beispiel des Bundeslandes Wien bedeutet das eine mögliche Erhöhung der mittleren Anzahl an Hitzetagen von 11 Tagen (Referenzzeitraum 1971-2000) auf 17 (12 bis 25 Tage) oder 40 Tage (30 bis 73 Tage; Ensemblemedian und Bandbreite von RCP 2.6 bzw. 8.5) in der fernen Zukunft (2071-2100). Ohne globale und effektive Klimaschutzmaßnahmen würde das Rekordjahr 2019 (zweitwärmster Sommer der 250-jährigen Messgeschichte; 38 Hitzetage in Wien) am Ende des Jahrhunderts somit zu einem durchschnittlichen Jahr, im Extremfall sogar zu einem unterdurchschnittlichen. Einzeljahre mit deutlich mehr Hitzetagen sind dann möglich.

Aufgrund verschiedener Rückkopplungsprozesse (z. B. Schneealbedofeedback) und der rascheren Erwärmung von Land- gegenüber Ozeanflächen ist auch in Zukunft im Alpenraum mit einer höheren Empfindlichkeit des Klimasystems verglichen mit dem globalen Mittel zu rechnen, auch wenn die zukünftig erwartete Erwärmungsrate von $0,25^{\circ} \mathrm{C}$ bzw. $0,36{ }^{\circ} \mathrm{C}$ pro Jahrzehnt (nahe/ferne Zukunft) vermutlich unter dem sehr hohen beobachteten Wert der letzten vier Dekaden liegen wird (Gobiet et al. 2014).

\section{Niederschlag und Globalstrahlung}

Beim Niederschlag sind die Änderungen oft nicht signifikant, da die natürlichen Schwankungen des Niederschlags

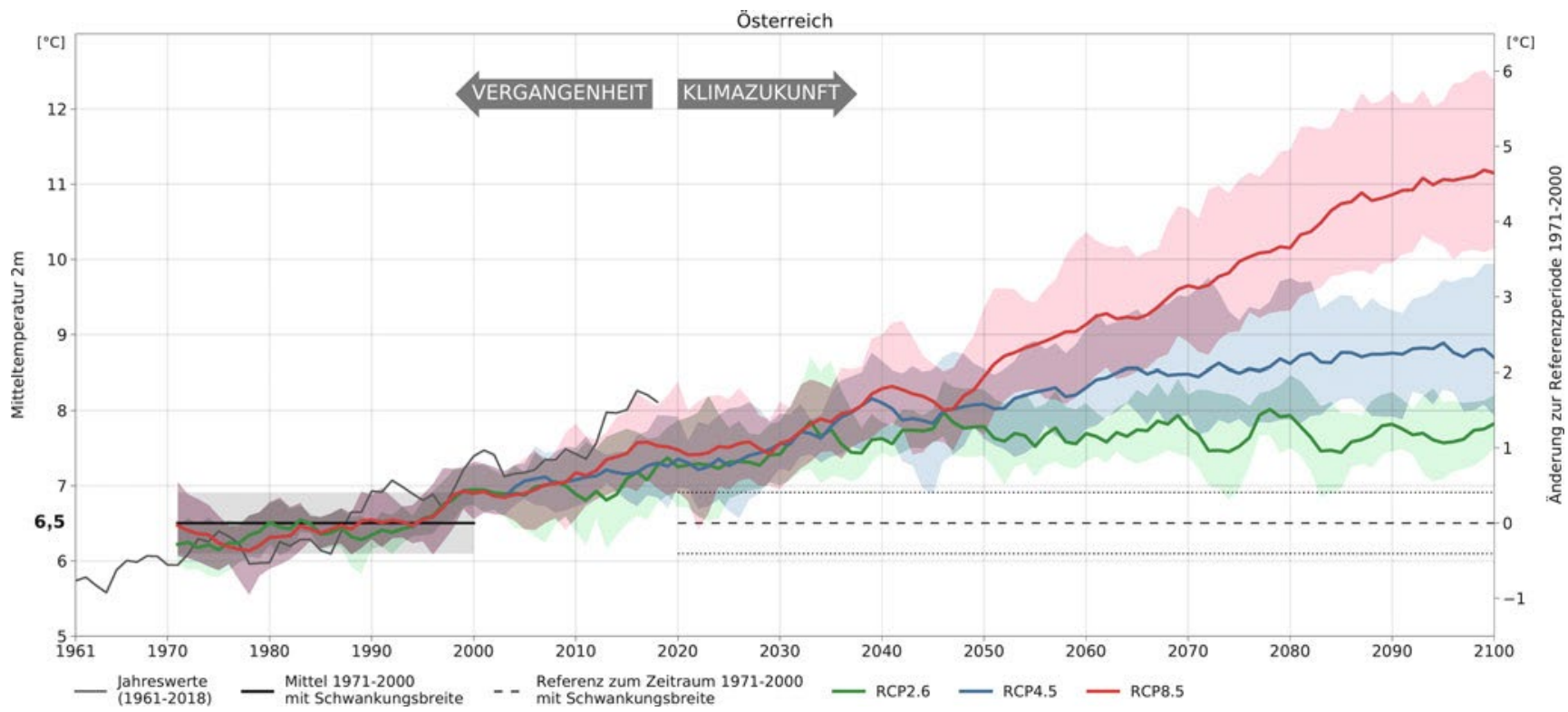

Abb. 2.7 Gemessene vergangene (1961-2018) und zukünftige erwartete (Modellberechnungen für RCP 2.6, RCP 4.5 und RCP 8.5 im Zeitraum 1970-2100) Jahresmitteltemperaturen für Österreich. Da Klimamodelle keine konkreten Vorhersagen für einzelne Jahre machen können, sind die Modellergebnisse und Messdaten geglättet dargestellt (5-jähriges gleitendes Mittel). Die Bandbreite der verschiedenen Klimamodelle ist pro Szenario durch die farbigen Bereiche angedeutet, die dicke farbige Linie zeigt jeweils den Median der Modelle. Die Variation von Jahr zu Jahr ist durch den schattierten Bereich angedeutet. (Chimani et al. 2016; aktualisiert) 

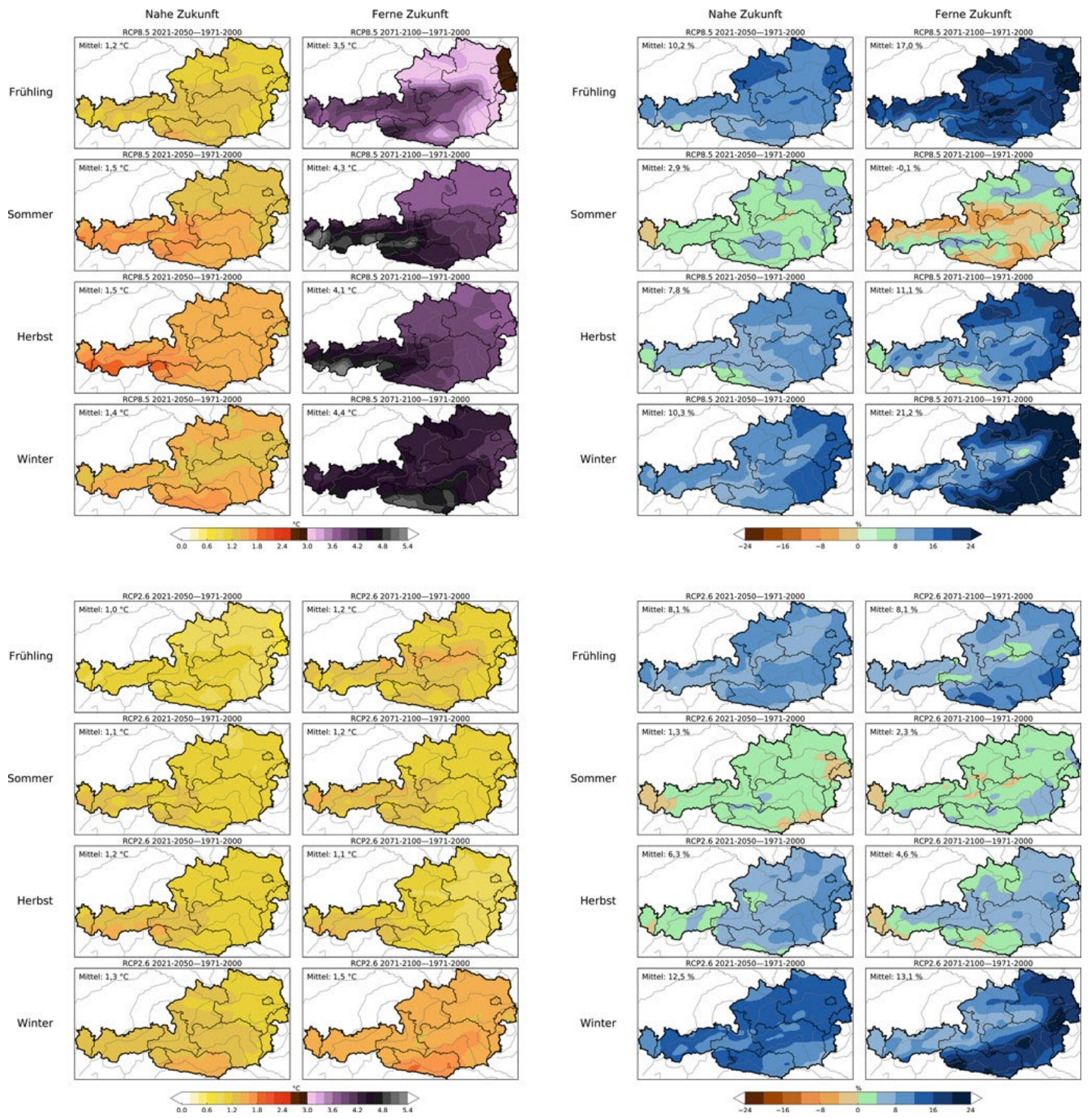

Abb. 2.8 Erwartete saisonale Temperatur- (links) und Niederschlagsänderungen (rechts) für Österreich in der nahen (2021-2050) und fernen (2071-2100) Zukunft im Verhältnis zur Periode 1971-2000 auf Basis der ÖKS15-Klimaszenarien (Chimani et al. 2016) für das Szenario RCP 8.5 („,business as usual“, oben) und RCP 2.6 („Paris-Ziel“, unten). Während den Temperaturänderungen hohes Vertrauen geschenkt werden kann, sind die Niederschlagsänderungen mit deutlich höheren Unsicherheiten behaftet bzw. teilweise auch nicht signifikant

in der Vergangenheit sehr groß sind und die Klimamodelle Niederschlag prinzipiell schlechter berechnen können als die Temperatur. Im saisonalen Vergleich zeigen sich nur im RCP- 8.5-Szenario der fernen Zukunft signifikante Änderungen für größere zusammenhängende Gebiete. Dies gilt im Winter vor allem für Nordostösterreich mit einer Zunahme von durchschnittlich $30 \%$ sowie im Frühling im Bereich der nördlichen Kalkalpen und dem nördlichen Alpenvorland mit einer Zunahme von rund 18 \%. Im Sommer zeigen sich bis auf wenige kleinräumige Ausnahmen keine signifikanten oder interpretierbaren Änderungen. Der Herbst liefert nur für kleinere Gebiete des nördlichen Alpenvorlands eine deutliche Änderung mit einer mittleren Zunahme von $18 \%$. Signifikante Änderungen der maximalen Tagesniederschläge 
in größeren zusammenhängenden Gebieten ergeben sich in beiden Szenarien erst in der fernen Zukunft und zeigen eine Zunahme im Jahresdurchschnitt an. Für die maximalen Mehrtagesniederschläge tritt eine Zunahme ebenso erst in der fernen Zukunft und ausschließlich in RCP 8.5 deutlich hervor. Die deutlichste Änderung ergibt sich im Winter in weiten Teilen Nord- und Ostösterreichs mit Zunahmen zwischen 20 und $40 \%$. Für Trocken- und Niederschlagsepisoden (eine zumindest fünf bzw. drei Tage andauernde durchgängige Periode an Tagen ohne/mit Niederschlag) liefern die Klimaszenarien keine interpretierbaren Änderungen, wobei die Niederschlagshäufigkeit im Sommerhalbjahr tendenziell abnimmt. Bei der Globalstrahlung zeigen sich die stärksten Abnahmen österreichweit im Winter und insbesondere im Flachland (RCP 4.5: $-4,8 \%$; RCP 8.5: $-8,4 \%$ ), ein ähnliches Signal zeigt sich im Frühjahr (hier aber eher in den Bergen), wohingegen die Änderungen im Sommer und Herbst nicht signifikant sind.

\section{Saisonale Schneedecke}

Generell sind Szenarien für die zukünftige Entwicklung der Schneelage vor allem in Höhenlagen aussagekräftig, in denen ein direkter Zusammenhang mit der Temperatur besteht (d. h. in tiefen und mittleren Lagen unterhalb von ca. $1500 \mathrm{~m}$ Seehöhe; Schöner et al. 2018; Olefs et al. 2019), denn die Temperatur gilt als zuverlässigster Parameter der Klimamodelle (Gobiet et al. 2014). Im Hochgebirge ist es im Winter hingegen ohnehin meistens kalt genug für Schneefall. Hier hängt die Schneelage auch in Zukunft mehr vom Niederschlag ab und damit von den Wetterlagen (Schöner et al. 2018). Zukunftsszenarien von Klimamodellen zeigen tendenziell mehr Niederschlag im Winter, was im Hochgebirge sogar zu mehr Schnee führen könnte (z. B. Gobiet et al. 2014). Diese Niederschlagsszenarien sind aber mit sehr großen Unsicherheiten behaftet.

Für den natürlichen Schnee ist in naher Zukunft (20212050) weiterhin mit einer hohen Jahr-zu-Jahr- und dekadischen Variabilität zu rechnen, die den langfristigen Trend überlagert und sich somit regional deutlicher bemerkbar machen kann als der allmähliche Anstieg der mittleren Temperatur (Roth 2018). Andererseits zeigen Studien aus der Schweiz eine Abnahme der mittleren Schneehöhe auch in naher Zukunft um -10 bis $-70 \%$ (hohe bzw. tiefe Lagen; Schmucki et al. 2015; Marty et al. 2017b). Für die ferne Zukunft zeigt sich eine klare Abnahme des Schneefalls von ca. $-20 \%$ bzw. $-40 \%$ (RCP 4.5 bzw. 8.5) im Mittel über alle Höhenstufen und den gesamten Alpenraum (Frei et al. 2018; NCCS 2018). Für die am Boden liegende Schneedecke in der fernen Zukunft werden gemittelt über die Schweiz Reduktionen um ca. 20, 50 und $70 \%$ (RCP 4.5; wirksame Klimaschutzmaßnahmen) bzw. 50, 70 und $90 \%$ (RCP 8.5, kein Klimaschutz) in hohen, mittleren und tiefen Höhenlagen erwartet (Schmucki et al. 2015; NCCS 2018; Kotlarski et al.
2018). Durch Erreichen des Paris-Ziels (RCP 2.6) würden sich diese Abnahmen auf ca. 10, 20 und $40 \%$ (hohe, mittlere, tiefe Lagen der europäischen Alpen) deutlich reduzieren lassen (IPCC 2019b). Je nach Szenario und Höhenlage verkürzt sich dabei die Schneedeckendauer um 2 Wochen bis 1 Monat am Winterbeginn und 1 bis 3 Monate am Ende des Winters (ca. Halbierung der Dauer in $1500 \mathrm{~m}$ Seehöhe ohne Klimaschutz; Marty et al. 2017b). Die Anforderungen an die technische Schneeproduktion werden im Alpenraum aufgrund der steigenden Lufttemperaturen insbesondere in tiefen und mittleren Höhenlagen bis ca. 1500 m stark steigen, da sich die Zeitfenster für die mögliche Schneeerzeugung (Annahme: heutige Technologie bzgl. Grenztemperaturen) deutlich reduzieren werden (Lehning et al. 2019; Spandre et al. 2019).

Im Rahmen des derzeit laufenden, vom ACRP geförderten Forschungsprojekts FuSE-AT wird die zukünftig erwartete Schneedeckenentwicklung für Österreich auf Basis von ÖKS15 (mit bzw. ohne Klimaschutzmaßnahmen) unter Berücksichtigung des Potenzials der technischen Beschneiung regional umfassend abgeschätzt. Detaillierte Projektergebnisse sind für das Jahr 2020 geplant.

\section{Permafrost und Gletscher}

Aufgrund der Wärmeübertragung ist davon auszugehen, dass sich die Bodentemperaturen in Bereichen des eisarmen Permafrosts langfristig um etwa denselben Betrag erhöhen werden wie die Lufttemperaturen. Des Weiteren ist mit fortschreitender zukünftiger Erwärmung auch von einer weiteren Vergrößerung der oberflächennahen Auftauschicht und Verkleinerung des flächigen Permafrosts auszugehen (APCC 2014; Gobiet et al. 2014; Biskaborn et al. 2019).

Aufgrund der erwarteten langfristig fortschreitenden Erwärmung und Trägheit der Anpassung an ein geändertes Klima werden die kleinen und mittleren Gletscher Österreichs bis zum Ende des 21. Jahrhunderts unabhängig vom Klimaszenario sehr wahrscheinlich verschwunden sein, die großen, in stark verkleinerter Form, das 22. Jahrhundert noch erleben (Größe abhängig vom Szenario). Die österreichischen Gletscher werden aufgrund der geringeren Gipfelhöhen früher abschmelzen als die im Mittel höher gelegenen Gletscher der Westalpen (Böhm et al. 2007; Olefs et al. 2009; APCC 2014).

Diese erwarteten Änderungen folgen dem fortschreitenden stark abnehmenden globalen Trend der Kryosphäre (IPCC 2019b).

\section{Wind}

Analysen von Klimaprojektionen zukünftiger Sturmtätigkeit über Zentraleuropa und Österreich zeigen kein eindeutiges Ergebnis bei der Änderung der Anzahl an Stürmen (in etwa gleich viele Studien, die von einer $\mathrm{Zu}$ - bzw. Abnahme ausgehen). Die Mehrzahl dieser Studien beschreibt jedoch im 
Zuge eines sich wandelnden Klimas eine Zunahme der Sturmintensität (Feser et al. 2015; Matulla et al. 2020; für kleinräumige Windböen in Zusammenhang mit Gewittern und Konvektion siehe nächster Abschnitt). Für kleinräumige, thermisch induzierte Windsysteme wie Hangwinde und Thermik sind auch für die Zukunft keine Untersuchungen bekannt.

\section{Starkniederschläge, Gewitter und Hagel}

Für die Abschätzung von zukünftigen Trends konvektiver Ereignisse (Gewitter, Hagel, kleinräumige Starkregen) ist man auf indirekte Methoden angewiesen, die bisher hauptsächlich auf Hagel angewandt wurden (Pistotnik et al. 2020). Auswertungen der ÖKS15-Klimamodelle des sog. Showalter-Index, der ein Maß für die Labilität der Luftschichtung ist, haben gezeigt, dass schwere Gewitter in Zukunft häufiger auftreten könnten, wobei die Zunahmen in der 2. Hälfte des 21. Jahrhunderts ohne Klimaschutzmaßnahmen deutlich stärker sind als mit wirksamen Maßnahmen (Formayer et al. 2018).

Das zukünftige Hagelpotenzial in Mitteleuropa zeigt anhand zweier Methoden, einer Anwendung von hagelauslösenden Größen auf das Gitter von Klimamodellen (Mohr et al. 2015) und einer Untersuchung der Häufigkeit hagelträchtiger Wetterlagen (Kapsch et al. 2012), eine statistisch insignifikante Zunahme für die nahe Zukunft (2021-2050). Für die ferne Zukunft (2071-2100) zeigt sich allerdings eine Zunahme des Hagelrisikos in weiten Teilen Europas, indem im Mittel instabilere Luftschichtungen eine schwächere vertikale Windscherung überkompensieren sollen, wobei diese $\mathrm{Zu}$ nahme im ausgehenden 21. Jahrhundert immer öfter statistisch signifikant werden soll (Marsh et al. 2009; Sander 2011; Púčik et al. 2017; Rädler et al. 2018). Belastbarere Aussagen zur zukünftigen Häufigkeit und Intensität von Gewittern werden erst mit der Verfügbarkeit hochqualitativer, konvektionsauflösender Klimamodelle in den nächsten Jahren möglich sein. Dennoch muss aus heutiger Sicht eher von einem Anstieg der Häufigkeit von schweren Gewittern in Österreich und den damit einhergehenden Problemen wie Hagel, Sturmböen, Blitzschlag, kleinräumigen Überflutungen und Murgängen ausgegangen werden.

\section{Klimatische Wasserbilanz}

Temperaturbedingt ist von einer weiteren Zunahme der Evapotranspiration (Verdunstung) auszugehen, die abhängig vom Klimaszenario insbesondere in tiefen Lagen der warmen Jahreszeit für moderate bis starke langfristige Abnahmen der Bodenfeuchte und somit vermehrte Dürreperioden sorgen wird (Haslinger et al. 2016; Reniu 2017). Extreme Dürreereignisse werden somit in Häufigkeit und Intensität im Laufe des 21. Jahrhunderts weiter zunehmen, ein derartiges Ereignis das im heutigen Klima im Schnitt alle 20 Jahre auftreten kann, wird am Ende des Jahrhunderts alle
2 bis 5 bzw. 5 bis 15 Jahre vorkommen (kein Klimaschutz (RCP 8.5) vs. wirksame Maßnahmen (RCP 4.5); Haslinger et al. 2016).

\section{Abfluss}

Prognosen über Hochwasseränderungen sind nach dem derzeitigen Kenntnisstand nicht möglich, da die zukünftige Entwicklung der Extremwerte des Klimas nicht ausreichend zuverlässig berechnet werden kann. Die natürliche Variabilität der Hochwässer ist wesentlich größer als die erwartete Änderung zufolge des Klimawandels (Blöschl et al. 2011; BMLFUW 2017). Bei den Niedrigwasserabflüssen ist in $\mathrm{Zu}$ kunft oberhalb $900 \mathrm{~m}$ Seehöhe mit einer weiteren Zunahme zu rechnen. Unterhalb $900 \mathrm{~m}$ Seehöhe und insbesondere in den Flachlandregionen Ost- und Südösterreichs wird mit einer weiteren Abnahme der Niedrigwasserabflüsse von 10 $15 \%$ für den Zeithorizont 2021 bis 2050 gerechnet (Blöschl et al. 2011; BMLFUW 2017). Bis Mitte des Jahrhunderts ist beim mittleren jährlichen Abfluss im Südosten Österreichs mit einer Abnahme zu rechnen, wohingegen im Rest des Landes keine statistisch signifikanten Trends zu erwarten sind (Blöschl et al. 2011).

\section{Sonstige touristisch relevante Klimafolgen}

Bereits für die nahe und in verstärkter Form auch für die ferne Zukunft wird mit einer Zunahme von durch den Klimawandel induzierten, niederschlagsbedingten Hangrutschungen im Ausmaß von 1 bzw. 14 Perioden pro Jahr mit unmittelbaren Auswirkungen auf die Straßen- und Eisenbahninfrastruktur gerechnet (Matulla et al. 2018b; Schlögl und Matulla 2018; Enigl et al. 2019). Mit der Zunahme von warmen und trockenen Witterungsphasen in den Sommermonaten ist auch eine Zunahme der Waldbrandhäufigkeit in den Alpen zu erwarten (Sass et al. 2014). Bezüglich der Wasserqualität ist zu erwarten, dass sich die anthropogenen Einflüsse (Verschmutzung, Sanierung) auch in Zukunft rascher bemerkbar machen werden, als sich die klimatischen Rahmenbedingungen ändern. Für Gewässer, die heute im Grenzbereich zwischen Zielzustand und ,mäßigem Zustand" liegen, besteht ein erhöhtes Risiko, infolge der bis 2050 erwarteten Auswirkungen des Klimawandels den ,guten Zustand" zu verfehlen, bei gering belasteten Gewässern wird der Einfluss gering sein. Temperaturerhöhungen in den Gewässern werden zu einer Anpassung der aquatischen Biozönosen führen, die Bioregionen werden sich daher verschieben (Blöschl et al. 2011).

\subsection{Auswirkungen auf touristische Klimaindizes}

Touristische Aktivitäten, die im Freien stattfinden, sind wetterabhängig und damit grundsätzlich anfällig für Aus- 


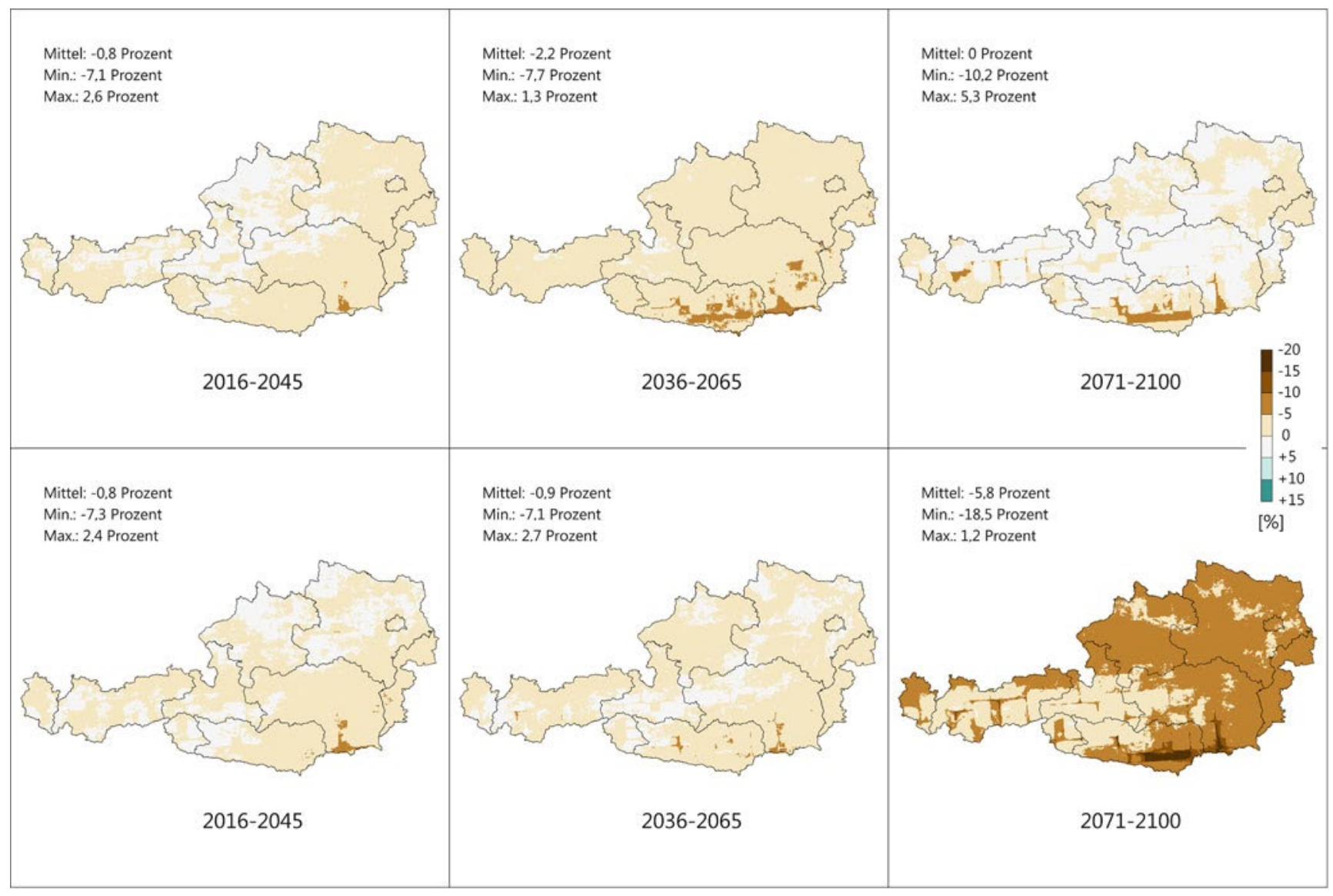

Abb. 2.9 Entwicklung der Niederschlagshäufigkeit im Sommerhalbjahr Ensemblemittel RCP 4.5 (oben) und RCP 8.5 (unten) für 2030 (links), 2050 (mittig) und Ende des 21. Jahrhunderts (rechts). In allen Zeitscheiben und 3 Szenarien kommt es zu einer Abnahme der Niederschlagshäufigkeit. (Datenquelle: CLIMA-MAP, Hohenwallner et al. 2018; Grafik: Herbert Formayer und Benedikt Becsi)

wirkungen des Klimawandels (Steiger 2016). Dabei können unterschiedliche Faktoren eine Rolle spielen:

- Umweltvoraussetzung für Aktivitäten (z. B. Schnee zum Skifahren, Wind zum Segeln, badetaugliche Wassertemperaturen, Erreichbarkeit der Urlaubsdestination),

- Wohlbefinden der Akteure (z. B. Hitzebelastung bei Städtereisen, badetaugliche Witterung, Regenperioden bei Wanderungen),

- Gefahr für Leib und Leben durch extreme Wetterereignisse (z. B. Sturm, schwere Gewitter, Lawinenabgänge).

Dabei können die aktuellen Wetterabläufe relevant sein oder die Vorbedingungen, welche sich über mehrere Wochen und Monate hin entwickeln. Dementsprechend unterschiedlich müssen auch Indikatoren definiert sein. Um aktuelle Wetterabläufe zu repräsentieren, werden häufig Schwellwertüberschreitungen auf Tagesbasis wie etwa Hitzetage (Tmax $\geq 30^{\circ} \mathrm{C}$ ) oder Niederschlagstage (Tagesniederschlag $\geq 1 \mathrm{~mm}$ ) verwendet. Es kann auch die Kombination von mehreren Variablen sein, wie etwa bei der „physiologischen äquivalenten Temperatur“" (PET; Matz- arakis 2007), bei der versucht wird die Energiebilanz eines Menschen abzubilden.

Bei kumulierenden Größen können einerseits einfache saisonale oder monatliche Veränderungen etwa der Mitteltemperatur im Frühjahr oder Sommer verwendet werden, um zum Beispiel die Verlängerung oder Verschiebung der Badetauglichkeit von Gewässern darzustellen. Bei der Schneesicherheit müssen hingegen komplexere Ansätze wie Schneemodelle auf Tagesbasis, wenn möglich mit Berücksichtigung von technischer Beschneiung, verwendet werden, um der Komplexität des Schneedeckenauf-/-abbaus sowie den besonderen Bedingungen auf Skipisten gerecht zu werden (Hofstätter und Formayer 2011 bzw. Hanzer et al. 2014; s. auch Abschn. 6.1).

Neben rein meteorologisch definierten Indikatoren gibt es auch Indikatoren, welche versuchen sozioökonomische Kenngrößen, wie die Bedingungen in Quell- und Zielgebieten, sowie die Größe von Tourismusströmen mit klimatischen Kennzahlen zu verknüpfen. Hierfür werden häufig Regressionsmodelle verwendet (z. B. Agnew und Palutikof 2001; Maddison 2001; Lise und Tol 2002). Diese Indikatoren sind eher für globale Betrachtungen geeignet und weniger für die Darstellung der touristischen Eignung innerhalb von Öster- 


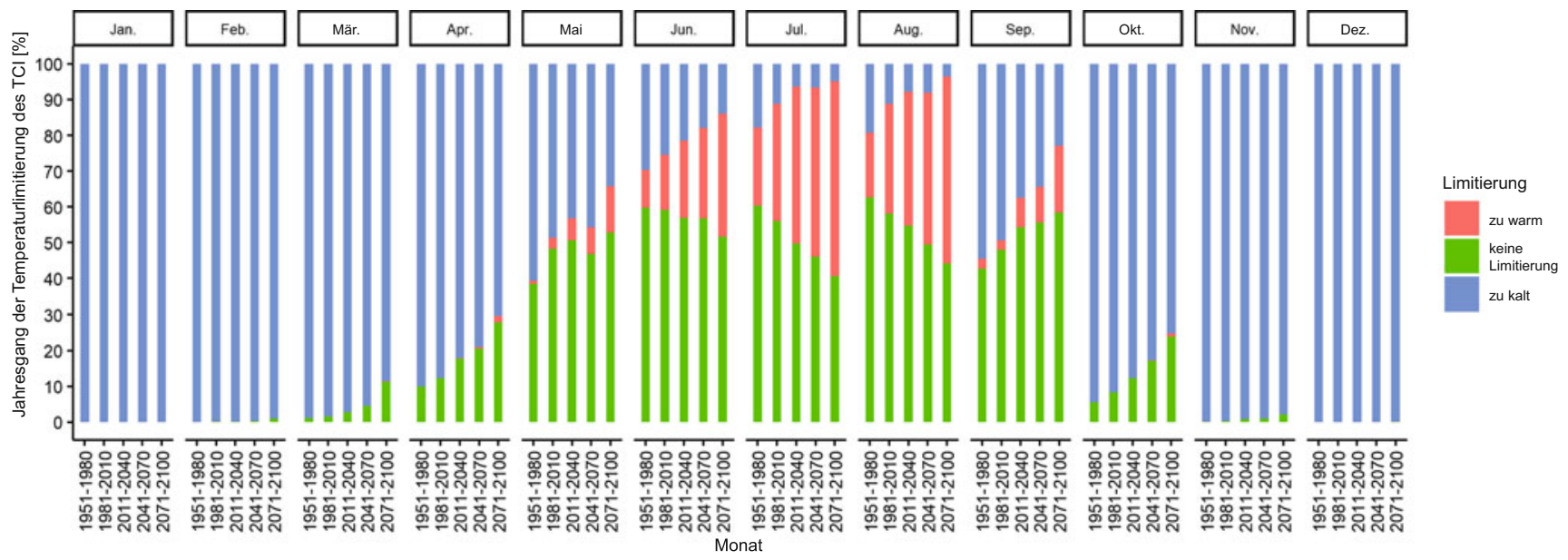

Abb. 2.10 Jahresgang der Temperaturlimitierung des Tourism Climatic Index (TCI) für Wien Hohe Warte beobachtet und mittleres RCP-8.5Szenario für das 21. Jahrhundert (Datenquelle: ZAMG). Durch diese TCI-Auswertung können quantitativ die Verbesserung der thermischen Bedingungen durch den Klimawandel in Wien für Freiluftaktivitäten in den Übergangsjahreszeiten sowie eine Verschlechterung in den Sommermonaten gezeigt werden. Der TCI ist jedoch nicht geeignet Freiluftaktivitäten in den Wintermonaten zu bewerten. (Grafik: Herbert Formayer)

reich sowie der klimawandelbedingten Veränderungen, die in diesem Kapitel vorwiegend diskutiert werden. Daher wird hier nicht näher auf diese eingegangen, obwohl sie für Untersuchungen der Attraktivität der Alpen als kühle Sommerdestination auf europäischer Skala durchaus geeignet sind.

Eine Vielzahl an klassischen klimatologischen Indikatoren wurde im Rahmen von ÖKS15 (Chimani et al. 2016) sowie CLIMA-MAP (Hohenwallner et al. 2018) für Österreich mit hoher räumlicher Auflösung sowohl historisch anhand von Beobachtungsdaten als auch für das ganze 21. Jahrhundert berechnet. Exemplarisch ist in Abb. 2.9 die Veränderung der Häufigkeit von Tagen mit Niederschlag im Sommerhalbjahr dargestellt, wie sie für das Projekt CLIMA-MAP berechnet wurde. Für das RCP 4.5 und RCP 8.5 wurde aus allen Modellen der Median des Änderungssignals berechnet und für die nahe, mittlere und ferne Zukunft dargestellt. In allen Zeitscheiben und beiden Emissionsszenarien kommt es im Sommerhalbjahr zu einer Abnahme der Niederschlagshäufigkeit. Am stärksten ist die Änderung am Ende des 21. Jahrhunderts, wenn keine Klimaschutzmaßnahmen umgesetzt werden. Dies führt landesweit zu einer Abnahme der Niederschlagstage um rund $6 \%$. Für touristische Freiluftaktivitäten im Sommerhalbjahr bedeutet dies meist eine Verbesserung, da niederschlagsfreie Tag besser für Baden, Radfahren oder Wandern geeignet sind. Lediglich bei Städtereisen während des Hochsommers könnte dies zu einer verstärkten Hitzebelastung und damit zu einer Reduktion der Attraktivität dieser Tourismussparte führen (Nicholls 2006).

\subsubsection{Touristische Indizes im Zusammenhang mit dem Klimawandel}

Um die Beziehung zwischen dem Klima und dem Tourismus herzustellen, wurden in der Vergangenheit diverse Indizes entwickelt. Eine Zusammenstellung tourismusrelevanter Klimaindikatoren bzw. Tourismusmodelle für Österreich ist in Tab. 2.1 wiedergegeben, jedoch ohne Anspruch auf Vollständigkeit. Die Liste kann mit faktisch allen klassischen klimatischen Indikatoren für Temperatur, Niederschlag, Sonnenscheindauer oder Schneedecke erweitert werden.

Nachstehend wird am Beispiel des TCI untersucht, ob und inwieweit der Klimawandel und sich ändernde klimatische Parameter Auswirkungen auf diese Indizes haben:

Ein Indikator, der gezielt den thermischen Komfort berücksichtigt ist der Tourism Climatic Index (TCI; Mieczkowski 1985; Amelung und Viner 2006). Der thermische Komfort wird hierbei als Funktion der Temperatur und der Luftfeuchtigkeit angesetzt. Zusätzlich fließen auch noch die tägliche Niederschlagsmenge, die mittlere Windgeschwindigkeit und die Sonnenscheindauer ein. Abweichend von der ursprünglichen Version, welche aus Monatsdaten berechnet wird, werden hier Tagesdaten zur Berechnung verwendet, wodurch wesentliche Limitierungen des TCI überwunden werden. In Abb. 2.10 ist der Jahresgang des TCI für die Station Wien Hohe Warte dargestellt, sowohl historisch als auch für ein Klimaszenario, das dem Ensemblemittel der RCP-8.5-Szenarien von ÖKS15 (Chimani et al. 2016) entspricht. Diese Auswertungen wurden extra für diesen Bericht gemacht, daher sind keine Referenzen angegeben. Es wurde die Limitierung des TCI aufgrund der Temperaturverhältnisse untersucht, wobei differenziert wurde, ob die Temperatur zu niedrig oder zu hoch war. Historisch werden die Klimaperioden 1951-1980 sowie 1981-2010 betrachtet, für die Zukunft die Perioden 2010-2040, 2041-2070 und 20712100. Historisch ist der TCI in Wien in den Wintermonaten November bis Februar ausschließlich durch zu niedrige Temperaturen limitiert. Im März treten erstmals temperaturunlimitierte TCI-Werte auf. Erst im Mai kommen historisch zu 
Tab. 2.1 Zusammenstellung von tourismusrelevanten Indikatoren

\begin{tabular}{|c|c|c|}
\hline Indikator/Modelle & Definition (klimatische Komponenten) & Quelle \\
\hline $\begin{array}{l}\text { Schneesicherheit, Verfügbarkeit von } \\
\text { Naturschnee }\end{array}$ & $\begin{array}{l}100 \text { Tage mit Schneedecke } \geq 30 \mathrm{~cm} \\
\text { zwischen } 16.12 \text {. und } 15.04 .\end{array}$ & Abegg (1996); Landauer et al. $(2012,2014)$ \\
\hline Optimale Reisetemperatur (Deutschland) & $21,53 \pm 1,6^{\circ} \mathrm{C}$ Tagesmitteltemperatur & Agnew und Palutikof (2001) \\
\hline Pooled Travel Cost Model & $\begin{array}{l}\text { Mittleres saisonales Temperaturmaximum } \\
{\left[{ }^{\circ} \mathrm{C}\right] \text {, saisonale Niederschlagssumme }[\mathrm{mm}]}\end{array}$ & Maddison (2001) \\
\hline Tourist Destination Model & $\begin{array}{l}\text { Monatsmitteltemperatur des wärmsten Mo- } \\
\text { nats, Niederschlagssumme des Sommers } \\
\text { (JJA) }\end{array}$ & Lise und Tol (2002) \\
\hline Schneesicherheit auf Skipisten & $\begin{array}{l}\text { Naturschnee und Kunstschnee auf Piste } \\
\geq 150 \mathrm{~mm} \text { Wasseräquivalent }\end{array}$ & Prettenthaler und Formayer (2011) \\
\hline Wanderwetter & $\begin{array}{l}\text { Tagestemperaturmaximum in } 1500 \text { masl } \\
\geq 15^{\circ} \mathrm{C} \text { an zumindest } 3,5,7 \text { zusammen- } \\
\text { hängenden Tagen }\end{array}$ & Prettenthaler und Formayer (2011) \\
\hline $\begin{array}{l}\text { Wahrscheinlichkeit für Schlechtwetter- } \\
\text { einbruch }\end{array}$ & $\begin{array}{l}\text { Tagestemperaturmaximum in } 1500 \text { masl } \\
<0{ }^{\circ} \mathrm{C} \text { und Tagesniederschlag } \geq 5 \mathrm{~mm}\end{array}$ & Prettenthaler und Formayer (2011) \\
\hline $\begin{array}{l}\text { Rocky Mountain National Park Be- } \\
\text { sucherschätzung }\end{array}$ & $\begin{array}{l}\text { Tagestemperaturmaximum }>80^{\circ} \mathrm{F} \\
\left(26,7{ }^{\circ} \mathrm{C}\right) \text {; Tagestemperaturminimum } \\
<10{ }^{\circ} \mathrm{F}\left(-12,2^{\circ} \mathrm{C}\right) \text {; Tagesniederschlag } \\
>0,25 \text { in }(6,35 \mathrm{~mm})\end{array}$ & Richardson und Loomis (2004) \\
\hline Urlaubserlebnis im Hochgebirge & Gletscher- und Permafrostrückgang & Pröbstl und Damm (2009) \\
\hline Tourism Climatic Index (TCI) & $\begin{array}{l}\text { Tagesmaximumtemperatur, Tagesmitteltem- } \\
\text { peratur, Minimum und Mittel der relativen } \\
\text { Luftfeuchte, Tagesniederschlag, Sonnen- } \\
\text { scheindauer, mittlere Windgeschwindigkeit }\end{array}$ & $\begin{array}{l}\text { Amelung und Viner (2006); Scott et al. } \\
\text { (2004) }\end{array}$ \\
\hline Holiday Climate Index (HCI) & $\begin{array}{l}\text { Tagesmaximumtemperatur, relative Luft- } \\
\text { feuchte, Tagesniederschlag, Bewölkung, } \\
\text { Windgeschwindigkeit }\end{array}$ & Scott et al. (2016) \\
\hline $\begin{array}{l}\text { Physiologische äquivalente Temperatur } \\
\text { (PET) }\end{array}$ & $\begin{array}{l}\text { Temperatur, Strahlungstemperatur, Luft- } \\
\text { feuchtigkeit, Wind; versucht den Wärme- } \\
\text { haushalt eines Menschen abzubilden }\end{array}$ & Matzarakis (2007) \\
\hline Badetage & $\begin{array}{l}\text { Tagesmaximumtemperatur }>23^{\circ} \mathrm{C} \text {; } \\
\text { Sonnenscheindauer }>9 \mathrm{~h}\end{array}$ & CLIMA-MAP (Hohenwallner et al. 2018) \\
\hline Hitzetage & Tagesmaximumtemperatur $\geq 30^{\circ} \mathrm{C}$ & CLIMA-MAP (Hohenwallner et al. 2018) \\
\hline Sommertage & Tagesmaximumtemperatur $\geq 25^{\circ} \mathrm{C}$ & CLIMA-MAP (Hohenwallner et al. 2018) \\
\hline Tropennächte & Tagesminimumtemperatur $\geq 20^{\circ} \mathrm{C}$ & CLIMA-MAP (Hohenwallner et al. 2018) \\
\hline Nasse Tage & Tagesniederschlag $\geq 1 \mathrm{~mm}$ & CLIMA-MAP (Hohenwallner et al. 2018) \\
\hline
\end{tabular}

hohe Temperaturen vor, diese erreichen im Juli und August ihr Maximum, nehmen im September stark ab und kommen im Oktober nicht mehr vor.

Bereits zwischen den beiden historischen Perioden zeigen sich klare Veränderungen. So nehmen in den Monaten Mai und September die thermisch nicht limitierten TCI-Werte in der 1981-bis-2010-Periode deutlich zu. Im Juli und August hingegen nehmen die nichtlimitierten TCI-Werte ab, da die Limitierung durch zu hohe Temperaturen zunimmt. Waren in der Klimaperiode 1951-1980 im Juli und August die durch zu hohe und zu niedrige Temperaturen limitierten Werte mit rund
$20 \%$ noch etwa gleich hoch, so liegen diese in der 1981-bis2010-Periode bei rund $15 \%$ bei zu niedrigen Temperaturen und rund $30 \%$ bei zu hohen Temperaturen. Bereits heute sind also in Wien im Sommer zu heiße Tage schon ein häufigeres Problem als zu kalte Tage.

Diese bereits beobachtete Entwicklung setzt sich in den Szenarien fort. In den Übergangsmonaten März bis Mai und September bis November nehmen die unlimitierten TCIWerte kontinuierlich zu. Von Juni bis August nehmen die Limitierungen durch zu hohe Temperaturen stärker zu, als die Limitierungen durch zu kalte Temperaturen abnehmen. 
Dadurch sinkt in Wien in diesen Monaten die Anzahl der thermisch nichtlimitierten TCI-Werte von historisch rund $60 \%$ auf etwa $40 \%$ am Ende des 21. Jahrhunderts.

\subsubsection{Analyse möglicher Albedoeffekte}

In einer Studie von Joanneum Research (Schwaiger et al. 2017) wurde mit einem einfachen Strahlungsmodell versucht, den Kühleffekt von technischer Beschneiung aufgrund der Erhöhung der Albedo zu quantifizieren. Die Reduktion der aufgenommenen Sonnenstrahlung wurde der Erwärmung durch die bei der Produktion des technischen Schnees freigesetzten Treibhausgase gegenübergestellt. Die Studie kam zu dem Ergebnis, dass technische Beschneiung in Summe zu einer Abkühlung führt und damit dem anthropogenen Klimawandel entgegenwirkt. Zentral für die Ergebnisse dieser stark umstrittenen Studie ist die Wirkung der technisch beschneiten Pisten auf den Gesamtstrahlungshaushalt eines Skigebietes. In einer Studie des Forschungsprogrammes StartClim (Weihs und Laimighofer 2019) wurde die Methodik der Studie von Joanneum Research mit einem komplexen 3-D-Strahlungsmodell, unter Einbeziehung hochaufgelöster Gelände- und Landnutzungsdaten, sowie einer realistischen natürlichen Schneedecke verglichen. Dabei stellte sich heraus, dass die einfache Strahlungsmodellierung zu einer drastischen Überschätzung der Gebietsalbedo und damit des Abkühlungseffekts führt. Für den Monat April, den Monat mit der stärksten Kühlwirkung, ergibt die einfache Methodik eine Reduktion der aufgenommenen Sonnenstrahlung im Mittel um 14,7 W/ $\mathrm{m}^{2}$, die realistische Modellierung jedoch nur 2,3 W/m². Die Kühlwirkung wird daher bei einer einfachen Strahlungsmodellierung um den Faktor 6 überschätzt, da etwa Mehrfachreflexionen und damit die Absorption an Gegenhängen sowie der „Canyoneffekt“" von Bäumen an den Pistenrändern nicht berücksichtigt werden. Die realistische Berechnung der „Kühlwirkung“ durch technische Beschneiung führt zu deutlich geringeren Werten als in der Studie von Joanneum Research angenommen. Dadurch wird es sehr unwahrscheinlich, dass eine Bilanzierung mit dem Erwärmungseffekt durch den Treibhausgasausstoß aus der technischen Beschneiung letztlich zu einer Abkühlung führt.

\subsection{Handlungsoptionen, Kommunikations- und Forschungsbedarf}

Großer Forschungsbedarf herrscht beim generellen Zusammenhang zwischen dem Klimawandel und der atmosphärischen Zirkulation. Es gibt z. B. Anzeichen dafür, dass Wetterlagen mit der globalen Erwärmung tendenziell immer länger andauern (erhöhte Persistenz von Hitzewellen mit Trockenheit, Kaltlufteinbrüchen, Starkschneefällen etc.) und somit eine Saison prägen können (z. B. Mann et al. 2017; Coumou et al. 2018; Francis et al. 2018; Kornhuber et al. 2019). In diesem Zusammenhang scheint auch die Berücksichtigung der stratosphärischen Ozonchemie bei der Wechselwirkung zwischen arktischem Meereis und der atmosphärischen Zirkulation eine wichtige Rolle zu spielen (Romanowsky et al. 2019). Des Weiteren gilt es, die Ursachen und Dynamiken von Extremereignissen und deren statistische Einordnung besser verstehen zu lernen. Aussagen zur mittleren Temperaturentwicklung sind relativ zuverlässig. Aussagen zur Niederschlagsentwicklung sind dagegen mit großen Unsicherheiten behaftet. Hier besteht dringender Bedarf, die Datenlage zu verbessern, vor allem für höher gelegene Gebirgsregionen. Um die Niederschlagsentwicklung zuverlässiger einschätzen zu können, sind weitere Erkenntnisse über den Prozess der Niederschlagsbildung insbesondere im Gebirge notwendig. Deutliche Verbesserungen bei den saisonalen und dekadischen Wetter- und Klimaprognosen sind Voraussetzung zur Einschätzung der künftigen, kurz- und mittelfristigen Variabilität der Schneedecke oder anderer Parameter im Klimasystem. Ein deutliches Fragezeichen ergibt sich auch aufgrund der weiterhin steigenden Sonnenscheindauer bei markant rückläufigem Luftdruck im Alpenraum (Auer et al. 2014). Eine vollständige Zusammenfassung des Forschungsbedarfs findet sich im APCC Science Plan - Zur strategischen Entwicklung der Klimaforschung in Österreich (CCCA 2018).

\subsection{Zusammenfassung}

Die Ergebnisse sind in der Abb. 2.11 zusammenfassend dargestellt. Angegeben wird in diesem Zusammenhang auch die Verlässlichkeit der getroffenen Aussagen.

Die in Abb. 2.11 dargestellten und bewerteten Wirkungen der klimainduzierten Phänomene werden im Folgenden ausführlich beschrieben.

- Die Lufttemperatur ist in Österreich seit Ende des 19. Jahrhunderts in allen Höhenlagen und Saisonen langfristig um ca. $2{ }^{\circ} \mathrm{C}$ angestiegen $\left(+0,25^{\circ} \mathrm{C}\right.$ pro Jahrzehnt). Dies entspricht einem doppelt so starken Anstieg im Vergleich zum mittleren globalen Anstieg und liegt $20 \%$ über dem Anstieg der mittleren Lufttemperatur von $1,5^{\circ} \mathrm{C}$ über den globalen Landflächen. Der langfristige Anstieg ist insbesondere im Winter überlagert von starken natürlichen Jahr-zu-Jahr- und multidekadischen Schwankungen (hohe Übereinstimmung, starke Beweislage).

- Für die nahe Zukunft (2021-2050) wird unabhängig vom Emissionsszenario ein weiterer Temperaturanstieg von ca. $1,3{ }^{\circ} \mathrm{C}$ im Vergleich zur Klimanormalperiode 1971-2000 erwartet (hohe Übereinstimmung, starke Beweislage). 
Veränderung klimainduzierter Phänomene mit Folgen für den Tourismus in Österreich im Laufe des 21. Jahrhunderts

\begin{tabular}{|c|c|c|c|c|}
\hline Klimainduzierte Phänomene & Indikator & Mögliche Folgen für den Tourismus & Klimaänderungssignal & Ausmaß der Veränderung \\
\hline Temperaturschwankung & Frost/Tau Wechsel & $\begin{array}{l}\text { Materialermüdung, Infrastruktur, } \\
\text { Straßenglätte (Kapitel 3) }\end{array}$ & $\Rightarrow$ & 0 \\
\hline Hitze & Hitzetage & $\begin{array}{c}\text { Hitzebelastung (Kapitel 9, 10); erhöhter } \\
\text { Energiebedarf für Klimatisierung (Kapitel 4, } 5 \text {, } \\
\text { 8, 10), Risiken bei Freizeitaktivitäten (Kapitel } \\
\text { 7) }\end{array}$ & ৯ & 3 \\
\hline Mittlere Temperaturänderung & $\begin{array}{l}\text { Jahres-/saisonale } \\
\text { Mittelwerte }\end{array}$ & $\begin{array}{l}\text { Alpine Gefahren durch Permafrost- und } \\
\text { Gletscherrückgang, Tauglichkeit für } \\
\text { Freiluftaktivitäten, Schneesicherheit (Kapitel } \\
6,7 \text { ) }\end{array}$ & $\gtrsim$ & 3 \\
\hline Kältewelle & $\begin{array}{c}\text { Eistage, Frosttage, } \\
\text { Heizgradtag }\end{array}$ & Wohlbefinden, Heizbedarf (Kapitel 4, 5, 6) & $\S$ & 2 \\
\hline $\begin{array}{c}\text { Großräumige } \\
\text { Starkniederschläge }\end{array}$ & $\begin{array}{l}\text { 3- bzw. 5-tägige } \\
\text { Niederschlagssummen }\end{array}$ & $\begin{array}{l}\text { Hochwasser, Erdrutsche, Gefährdung von } \\
\text { Infrastruktur, Verkehrseinschränkungen, } \\
\text { mehrtägiges Schlechtwetter; Auswirkung auf } \\
\text { Gebäude (Schäden), Gästeausfall, Risiken bei } \\
\text { Freizeitaktivitäten (Kapitel 4, 5, 6, 7) }\end{array}$ & $\Rightarrow$ & 0 \\
\hline Lokale Starkniederschläge & $\begin{array}{l}\text { 1-tägige (besser } \\
\text { stündliche } \\
\text { Niederschlagssumme, } \\
\text { Blitzaktivität) }\end{array}$ & $\begin{array}{l}\text { Lokale Hochwasser, Muren, Gefährdung von } \\
\text { Infrastruktur, Verkehrseinschränkungen, } \\
\text { Lebensmittelbeschaffung (Preisanstieg, } \\
\text { erhöhte Importe), Risiken bei } \\
\text { Freizeitaktivitäten (Kapitel } 4,5,6,7 \text { ) }\end{array}$ & $\gtrsim$ & 3 \\
\hline $\begin{array}{l}\text { Trockenheit/ } \\
\text { Trockenperioden }\end{array}$ & $\begin{array}{c}\text { Anzahl an } \\
\text { zusammenhängenden } \\
\text { Tagen ohne } \\
\text { Niederschlag; } \\
\text { klimatologische } \\
\text { Wasserbilanz }\end{array}$ & $\begin{array}{l}\text { Lebensmittelbeschaffung (Preisanstieg, } \\
\text { erhöhte Importe; Kapitel 4, 5), Auswirkung auf } \\
\text { Vegetation, Bewässerungsbedarf (z.B. Golf), } \\
\text { Wald/Flurbrände, Trinkwasserversorgung, } \\
\text { (Kapitel 6, 7) }\end{array}$ & $\gtrsim$ & 3 \\
\hline Schnee & $\begin{array}{l}\text { Schneehöhe, } \\
\text { Schneedeckendauer, } \\
\text { Neuschneesumme }\end{array}$ & $\begin{array}{l}\text { Schneesicherheit, Lawinengefahr, } \\
\text { Verkehrsbehinderung, Schneelast auf Dächer, } \\
\text { (Kapitel 4, 5, 6) }\end{array}$ & $\Xi$ & 3 \\
\hline $\begin{array}{l}\text { Wind (kleinräumig - } \\
\text { Gewitterstürme) }\end{array}$ & $\begin{array}{l}\text { Windgeschwindigkeit, } \\
\text { Windspitzen, } \\
\text { Blitzaktivität }\end{array}$ & $\begin{array}{l}\text { Gefährdung durch umstürzende Bäume, } \\
\text { Infrastruktur (Dächer, Fenster), } \\
\text { Verkehrsbehinderung, Stopp von Seilbahnen, } \\
\text { Outdooraktivitäten (Kapitel 4, 5, 6, 7) }\end{array}$ & $\gtrsim$ & 3 \\
\hline $\begin{array}{l}\text { Wind (großräumig - } \\
\text { atlantische Stürme, Föhn) }\end{array}$ & $\begin{array}{l}\text { Windgeschwindigkeit, } \\
\text { Windspitzen }\end{array}$ & $\begin{array}{c}\text { Gefährdung durch umstürzende Bäume, } \\
\text { Verkehrsbehinderung, Stopp von Seilbahnen, } \\
\text { Outdooraktivitäten (Kapitel 4, 5, 6, 7) }\end{array}$ & $\Rightarrow$ & 0 \\
\hline \multirow{2}{*}{\multicolumn{2}{|c|}{ Zunehmende Sicherheit der Aussage }} & kung $\longrightarrow$ & Klimaänderungssi & \\
\hline & & $\begin{array}{ll} & 3 \\
\end{array}$ & $\Rightarrow$ & ৯ \\
\hline
\end{tabular}

Abb. 2.11 Veränderung klimainduzierter Phänomene mit Folgen für den Tourismus in Österreich im Laufe des 21. Jahrhunderts. (Grafik: Herbert Formayer und Marc Olefs)

- Für die ferne Zukunft (2071-2100) wird die Temperatur (Ensemblemedian aller Modelle) je nach Emissionsszenario um weniger als $2^{\circ} \mathrm{C}(\mathrm{RCP} 2.6$, ,Paris Lifestyle“ - tiefgreifende Klimaschutzmaßnahmen), ca. $2{ }^{\circ} \mathrm{C}$ (RCP 4.5, effektive Klimaschutzmaßnahmen) oder $4{ }^{\circ} \mathrm{C}$ (RCP 8.5 „business as usual“ - keine Klimaschutzmaßnahmen) im Vergleich zur Klimanormalperiode 1971-2000 ansteigen. Die mögliche Bandbreite ohne Klimaschutzmaßnahmen (RCP 8.5) liegt dabei zwischen $+3,6$ und $+5,8^{\circ} \mathrm{C}$ (hohe Übereinstimmung, starke Beweislage).

- Bei der Niederschlagsentwicklung in der Vergangenheit zeigen sich regionale Unterschiede in den letzten
150 Jahren (10-15 \% Zu- bzw. Abnahme in Westen bzw. Südosten Österreichs; hohe Übereinstimmung, starke Beweislage).

- Für die ferne Zukunft (2100) wird eine Zunahme des Niederschlags in Teilregionen im Winter im Vergleich zur Klimanormalperiode 1971-2000 erwartet (ca. 2040 \%), die Häufigkeit von Sommerniederschlägen nimmt tendenziell ab (hohe Übereinstimmung, schwache Beweislage).

- Die natürliche Schneedeckendauer und Schneehöhe haben insbesondere im Westen und Süden Österreichs seit den 1950er-Jahren langfristig signifikant in allen Höhenlagen abgenommen, diese Abnahme ist aber über- 
lagert durch starke Jahr-zu-Jahr- und multidekadische natürliche Klimaschwankungen, die es auch in Zukunft weiterhin geben wird. In diesem Zusammenhang haben sich ebenfalls die klimatologischen Rahmenbedingungen für die technische Schneeerzeugung geändert. Anzahl und Dauer der potenziellen Schneizeiten haben sich langfristig verringert (hohe Übereinstimmung, starke Beweislage).

- In der nahen Zukunft (2021-2050) überlagern die starken regionalen Jahr-zu-Jahr- und multidekadischen natürlichen Schwankungen des Klimasystems (Temperatur und Niederschlag) in allen Höhenlagen deutlich den Effekt der allmählich steigenden Temperaturen auf die Schneedecke. Diese Schwankungen können sich somit regional sogar stärker bemerkbar machen als der langfristig abnehmende Trend (hohe Übereinstimmung, schwache Beweislage).

- Für die ferne Zukunft (2071-2100) überwiegt in hohen Lagen der Temperaturanstieg gegenüber dem möglichen Anstieg der Winterniederschläge (hohe Übereinstimmung, starke Beweislage).

- Im schlimmsten Fall und ohne Klimaschutz (RCP 8.5) bedeutet das eine Abnahme der Schneehöhe um ca. 50, 70 und $90 \%$ in hohen, mittleren und tiefen Lagen. Mit wirksamen Klimaschutzmaßnahmen (RCP 4.5) kann diese Reduktion auf ca. 20, 50 und $70 \%$ gedämpft werden, bei globalem Erreichen des Paris-Ziels (RCP 2.6) würden sich diese Abnahmen auf ca. 10, 20 und $40 \%$ (hohe, mittlere, tiefe Lagen der europäischen Alpen) drastisch reduzieren lassen. Die Schneedeckendauer verringert sich somit um bis zu 1 Monat zu Beginn und bis zu 3 Monaten zum Ende der schneebedeckten Zeit (hohe Übereinstimmung, starke Beweislage).

- Die Anzahl und Dauer der potenziellen Zeiten für die technische Beschneiung reduzieren sich in diesem Zusammenhang, unter der Annahme heutiger Technologie (Grenztemperaturen), insbesondere in mittleren und tiefen Höhenlagen weiter (hohe Übereinstimmung, starke Beweislage).

- Die Sonnenscheindauer hat im Alpenraum in den letzten 130 Jahren um ca. $10 \%$ zugenommen (hohe Übereinstimmung, starke Beweislage).

- Alle in Österreich beobachteten Gletscher haben seit 1980 signifikant an Fläche und Volumen verloren (hohe Übereinstimmung, starke Beweislage).

- Temperaturextrema (z. B. Anzahl der Hitzetage) haben signifikant zugenommen, diese Zunahme wird abhängig vom Emissionsszenario in Zukunft weitergehen und auch die Intensität von Hitzewellen wird weiterhin zunehmen (hohe Übereinstimmung, starke Beweislage).

- Großräumige (flächige) Starkniederschläge zeigen in der Vergangenheit über ganz Mitteleuropa keine signifikanten Trends (hohe Übereinstimmung, mittlere Beweislage).
- Die Zahl der Tage mit starken bis extremen kleinräumigen (konvektiven) Niederschlägen hat zulasten der Zahl an Tagen mit schwachen bis moderaten Niederschlägen zugenommen (mittlere Übereinstimmung, mittlere Beweislage).

- Über die vergangene langfristige Entwicklung von konvektiven Extremereignissen (lokale Starkregen, Gewitter, Hagel) sind derzeit nur indirekte Aussagen möglich, dabei hat sich das Potenzial für deren Bildung erhöht (hohe Übereinstimmung, schwache Beweislage).

- Insbesondere für die ferne Zukunft (2071-2100) und ohne globale Klimaschutzmaßnahmen zeigt sich eine deutliche und signifikante Zunahme von konvektiven Extremereignissen (Risiko für lokale Starkregen, Gewitter und Hagel; hohe Übereinstimmung, mittlere Beweislage)

- Aufgrund der in der Zukunft weiter erwarteten Zunahme der Verdunstung ist mit häufigeren und intensiveren extremen Dürreereignissen im Laufe des 21. Jahrhunderts zu rechnen.

\section{Kernaussagen - Kapitel 2}

- Die Lufttemperatur ist in Österreich seit Ende des 19. Jahrhunderts um knapp $2{ }^{\circ} \mathrm{C}$ in etwa doppelt so stark angestiegen wie im globalen Mittel ( $+20 \%$ im Vergleich zu den globalen Landflächen). Ein weiterer Temperaturanstieg von $+1,3{ }^{\circ} \mathrm{C}$ bis zum Jahr 2050 (unabhängig vom Emissionsszenario) bzw. unter 2 bis $4{ }^{\circ} \mathrm{C}$ (tief greifende globale Emissionsreduktionen (Paris-Ziel) bzw. keine Maßnahmen) bis zum Jahr 2100 ist zu erwarten. Die Erwärmung geht einher mit einer Zunahme der Hitzebelastung (längere und intensivere Hitzewellen; hohe Übereinstimmung, starke Beweislage).

- Die natürliche Schneedeckendauer und Schneehöhe haben insbesondere im Westen und Süden Österreichs seit 1950 langfristig signifikant in allen Höhenlagen abgenommen. Abhängig von der Höhenlage muss mit einer weiteren langfristigen Abnahme im Ausmaß von 10-40 \% bzw. 50-90 \% (drastische globale Klimaschutzmaßnahmen bzw. keine Maßnahmen) bis ans Ende des 21. Jahrhunderts gerechnet werden. In diesem Zusammenhang werden sich auch die Anzahl und Dauer der potenziellen Zeitfenster für die technische Beschneiung bei der Annahme heutiger Grenztemperaturen in allen Höhenlagen weiter reduzieren (hohe Übereinstimmung, starke Beweislage).

- In der Vergangenheit hat sich das Potenzial für die Bildung von konvektiven Extremereignissen (Risiko für lokale Starkregen, Überflutungen, Gewitter und Hagel) erhöht. Insbesondere für die zweite Hälfte des 21. Jahrhunderts und ohne Klimaschutzmaßnahmen 
ist mit einer deutlichen Zunahme der Intensität und Häufigkeit solcher Ereignisse und des damit zusammenhängenden Potenzials für kleinräumige Überflutungen, Hangrutschungen und Muren zu rechnen (hohe Übereinstimmung, mittlere Beweislage).

- Aufgrund der in der Zukunft weiter erwarteten Zunahme der Verdunstung ist insbesondere ohne Klimaschutz mit häufigeren und intensiveren extremen Dürreereignissen im Laufe des 21. Jahrhunderts zu rechnen.

- Das Ausmaß dieser Klimarisiken in der zweiten Hälfte des Jahrhunderts ist dabei wesentlich durch den Menschen beeinflussbar (drastische und rasche globale Klimaschutzmaßnahmen sowie regionale und sektorale Anpassungsmaßnahmen).

\section{Literatur}

Abegg, B. (1996) Klimaänderung und Tourismus: Klimafolgenforschung am Beispiel des Wintertourismus in den Schweizer Alpen. vdf Hochschulverlag AG, Zürich, Schweiz.

Agnew, M.D. \& Palutikof, J.P. (2001) Impacts of climate on the demand for tourism. In: Matzarakis, A., de Freitas, C.R. (Hrsg.) Proceedings of the first international workshop on climate, tourism and recreation, S. 41-50. International Society of Biometeorology - Commission on Climate Tourism and Recreation, 5-10 Oktober 2001, Neos Marmaras, Chalkidiki, Griechenland.

Aigner, G., Gattermayr, W. \& Zenkl, C. (2018) Die Winter in Tirol seit 1895: eine Analyse amtlicher Temperatur- und Schneemessreihen. Innsbruck, Österreich. Online unter: https://www.zukunft-skisport.at/ site/assets/files/1019/studie_tirol_forum-zukunft-skisport_nov2018. pdf (letzter Zugriff: 25.03.2020).

Amelung, B. \& Viner, D. (2006) Mediterranean tourism: exploring the future with the tourism climatic index. Journal of SustainableTourism 14(4), 349-366. DOI: https://doi.org/10.2167/jost549.0

APCC (2014) Österreichischer Sachstandsbericht Klimawandel 2014 (AAR14). Austrian Panel on Climate Change (APCC), Verlag der Österreichischen Akademie der Wissenschaften (ÖAW), Wien, Österreich. Online unter: http://www.austriaca.at/APCC_AAR2014.pdf (letzter Zugriff: 24.03.2020).

APCC (2018) Österreichischer Special Report Gesundheit, Demographie und Klimawandel (ASR18). Austrian Panel on Climate Change (APCC), Verlag der Österreichischen Akademie der Wissenschaften (ÖAW), Wien, Österreich. Online unter: https://austriaca.at/APCC ASR18.pdf (letzter Zugriff: 24.05.2020).

Auer, I., Böhm, R. \& Mohnl, H. (1989) Klima von Wien: eine anwendungsorientierte Klimatographie. Magistrat der Stadt Wien, Österreich

Auer, I., Böhm, R., Jurkovic, A., Lipa, W., Orlik, A., Potzmann, R., Schöner, W., Ungersböck, M., Matulla, C., Briffa, K., Jones, P.D., Efthymiadis, D., Brunetti, M., Nanni, T., Maugeri, M., Mercalli, L., Mestre, O., Moisselin, J.M., Begert, M., Müller-Westermeier, G., Kveton, V., Bochnicek, O., Stastny, P., Lapin, M., Szalai, S., Szentimrey, T., Cegnar, T., Dolinar, M., Gajic-Capka, M., Zaninovic, K., Majstorovic, Z. \& Nieplova, E. (2007): HISTALP - historical instrumental climatological surface time series of the greater Alpine region 1760-2003. International Journal of Climatology 27(1), 17-46. https://doi.org/10.1002/joc.1377

Auer, I., Böhm, R. \& Schöner, W. (2009) The importance of high quality regional scientific information in coping with global climate change. In: World Meteorological Organization (WMO) (Hrsg.) Climate Sense, S. 179-183. Tudor Rose, Leicester, Vereinigtes Königreich. Online unter: https://library.wmo.int/doc_num.php?explnum_id=3066 (letzter Zugriff: 25.03.2020).

Auer, I., Foelsche, U., Böhm, R., Chimani, B., Haimberger, L., Kerschner, H., Koinig, K. A., Nicolussi, K. \& Spötl, C. (2014) Band 1, Kapitel 3: Vergangene Klimaänderung in Österreich. In: APCC (2014) Österreichischer Sachstandsbericht Klimawandel 2014, S. 227-300. Austrian Panel on Climate Change (APCC), Verlag der Österreichischen Akademie der Wissenschaften (ÖAW), Wien, Österreich. Online unter: http://www.austriaca.at/APCC_AAR2014.pdf (letzter Zugriff: 24.03.2020).

Beniston, M. (1997) Variations of snow depth and duration in the Swiss Alps over the last 50 years: links to changes in largescale climatic forcings. Climatic Change 36, 281-300. DOI: https://doi. org/10.1023/A:1005310214361

Beniston, M., Farinotti, D., Stoffel, M., Andreassen, L.M., Coppola, E., Eckert, N., Fantini, A., Giacona, F., Hauck, C., Huss, M., Huwald, H., Lehning, M., López-Moreno, J.-I., Magnusson, J., Marty, C., Morán-Tejéda, E., Morin, S., Naaim, M., Provenzale, A., Rabatel, A., Six, D., Stötter, J., Strasser, U., Terzago, S. \& Vincent, C. (2018) The European mountain cryosphere: a review of its current state, trends, and future challenges. The Cryosphere 12(2), 759-794. DOI: https:// doi.org/10.5194/tc-12-759-2018

Biskaborn, B.K., Smith, S.L., Noetzli, J., Matthes, H., Vieira, G., Streletskiy, D.A., Schoeneich, P., Romanovsky, V.E., Lewkowicz, A.G., Abramov, A., Allard, M., Boike, J., Cable, W.L., Christiansen, H.H., Delaloye, R., Diekmann, B., Drozdov, D., Etzelmüller, B., Grosse, G., Guglielmin, M., Ingeman-Nielsen, T., Isaksen, K., Ishikawa, M., Johansson, M., Johannsson, H., Joo, A., Kaverin, D., Kholodov, A., Konstantinov, P., Kröger, T., Lambiel, C., Lanckman, J-P., Luo, D., Malkova, G., Meiklejohn, I., Moskalenko, N., Oliva, M., Phillips, M., Ramos, M., Sannel, A.B.K., Sergeev, D., Seybold, C., Skryabin, P., Vasiliev, A., Wu, Q., Yoshikawa, K., Zheleznyak, M. \& Lantuit, H. (2019) Permafrost is warming at a global scale. Nature Communications 10(264). DOI: https://doi.org/10.1038/s41467-018-08240-4

Blöschl, G., Schöner, W., Kroiß, H., Blaschke, A.P., Böhm, R., Haslinger, K., Kreuzinger, N, Merz, R., Parajka, J., Salinas, J.L. \& Viglione, A. (2011) Anpassungsstrategien an den Klimawandel für Österreichs Wasserwirtschaft - Ziele und Schlussfolgerungen der Studie für Bund und Länder. Österreichische Wasser- und Abfallwirtschaft 63, 1-10. DOI: https://doi.org/10.1007/s00506-010-0274-2

Blöschl, G., Hall, J., Parajka, J., Perdigão, R.A.P., Merz, B., Arheimer, B., Aronica, G.T., Bilibashi, A., Bonacci, O., Borga, M., Canjevac, I., Castellarin, A., Chirico, G.B., Claps, P., Fiala, K., Frolova, N., Gorbachova, L., Gül, A., Hannaford, J., Harrigan, S., Kireeva, M., Kiss, A., Kjeldsen, T.R., Kohnová, S., Koskela, J.J., Ledvinka, O., Macdonald, N., Mavrova-Guirguinova, M., Mediero, L., Merz, R., Molnar, P., Montanari, A., Murphy, C., Osuch, M., Ovcharuk, V., Radevski, I., Rogger, M., Salinas, J.L., Sauquet, E., Šraj, M., Szolgay, J., Viglione, A., Volpi, E., Wilson, D., Zaimi, K. \& Živkovic, N. (2017) Changing climate shifts timing of European floods. Science 357(6351), 588-590. DOI: https://doi.org/10.1126/science.aan2506

BMLFUW (2017) Klimawandel in der Wasserwirtschaft - Follow up zur ZAMG/TU-Wien Studie (2011) Anpassungsstrategien an den Klimawandel für Österreichs Wasserwirtschaft im Auftrag von Bund und Ländern. Bundesministerium für Land- und Forstwirtschaft, Umwelt und Wasserwirtschaft (BMLFUW), Wien, Österreich. Online unter: https://www.bmlrt.gv.at/wasser/wasser-oesterreich/foerderungen/ trinkwasser_abwasser/aktuelle projekte/klimawandel_wasserwirtschaft.html (letzter Zugriff: 01.04.2020).

BMLRT (2019) Der Klimawandel erhöht auch die Wassertemperatur der Donau. Bundesministerium für Landwirtschaft, Regionen und Tourismus (BMLRT), Wien, Österreich. Online unter: https://www. bmlrt.gv.at/wasser/wasser-oesterreich/wasserkreislauf/wtdonau2017. html (letzter Zugriff: 09.05.2020). 
Boeckli, L., Brenning, A., Gruber, S. \& Noetzli, J. (2012) Permafrost distribution in the European Alps: calculation and evaluation of an index map and summary statistics. The Cryosphere 6(4), 807-820. DOI: https://doi.org/10.5194/tc-6-807-2012.

Böhm, R. (1979) Stadtentwicklung und Trend der Wärmeinselintensität. Archiv für Meteorologie, Geophysik und Bioklimatologie Serie B 27, 31-46. DOI: https://doi.org/10.1007/BF02245908

Böhm, R., Auer, I., Schöner, W. \& Hagen, M. (1998) Long alpine barometric time series in different altitudes as a measure for 19th/20th century warming. Preprints of the 8th Conference on Mountain Meteorology, 72-76. American Meteorlogical Society (AMS), Boston, MA, USA. Online unter: http://www.zamg.ac.at/histalp/download/ abstract/Boehm-etal-1998-F.pdf (letzter Zugriff: 01.04.2020).

Böhm, R. (2006) ALP-IMP (EVK-CT-2002-00148): Multi-centennial climate variability in the Alps based on instrumental data, model simulations and proxy data. Final report for RTD-project. Zentralanstalt für Meteorologie und Geodynamik (ZAMG), Wien, Österreich. Online unter: http://www.zamg.ac.at/ALP-IMP/ (letzter Zugriff: 01.04.2020).

Böhm, R., Schöner, W., Auer I., Hynek, B., Kroisleitner, C. \& Weyss, G. (2007) Gletscher im Klimawandel: vom Eis der Polargebiete zum Goldbergkees in den Hohen Tauern. Eigenverlag der Zentralanstalt für Meteorologie und Geodynamik (ZAMG), Wien, Österreich.

Böhm, R. (2012) Changes of regional climate variability in central Europe during the past 250 years. The European Physical Journal Plus 127(54). DOI: https://doi.org/10.1140/epjp/i2012-12054-6

CCCA (2018) Science Plan: zur strategischen Entwicklung der Klimaforschung in Österreich. Climate Change Centre Austria (CCCA), Wien, Österreich. Online unter: https://www.ccca.ac.at/fileadmin/00_DokumenteHauptmenue/03_Aktivitaeten/Science_Plan/ CCCA_Science_Plan_2_Auflage_20180326.pdf (letzter Zügriff: 25.03.2020).

Chimani, B., Heinrich, G., Hofstätter, M., Kerschbaumer, M., Kienberger, S., Leuprecht, A., Lexer, A., Peßenteiner, S., Poetsch, M.S., Salzmann, M., Spiekermann, R., Switanek, M. \& Truhetz, H. (2016) ÖKS15 - Klimaszenarien für Österreich. Daten, Methoden und Klimaanalyse. Projektendbericht. Online unter: https://data.ccca.ac.at/ dataset/a4ec86ca-eeae-4457-b0c7-78eed6b71c05 (letzter Zugriff: 01.04.2020).

Chimani, B., Matulla, C., Eitzinger, J., Hiebl, J., Hofstätter, M., Kubu, G., Maraun, D., Mendlik, T., Schellander-Gorgas, T. \& Thaler, S. (2019) Guideline zur Nutzung der ÖKS15-Klimawandelsimulationen sowie der entsprechenden gegitterten Beobachtungsdatensätze. Ergebnis des Projektes STARC-Impact, gefördert durch den Klimaund Energiefonds im Rahmen des Programms „Austrian Climate Research Programme“ (ACRP). Online unter: https://data.ccca.ac.at/ dataset/guideline-zur-nutzung-der-oeks15-klimawandelsimulationen-v02/resource/37729819-bf1a-4066-8909-d615f9a92728 (letzter Zugriff: 01.04.2020).

Coumou, D., Di Capua, G., Vavrus, S., Wang, L. \& Wang, S. (2018) The influence of Arctic amplification on mid-latitude summer circulation. Nature Communications 9(2959). DOI: https://doi.org/10.1038/ s41467-018-05256-8.

Dokulil, M.T. \& Donabaum, U. (2014) Phytoplankton of the Danube River: composition and long-term dynamics. Acta Zoologica Bulgarica Suppl. 7, 147-152. Online unter: http://www.acta-zoologica-bulgarica.eu/downloads/acta-zoologica-bulgarica/2014/supplement-7-147-152.pdf (letzter Zugriff: 01.04.2020).

Ehlers, J. \& Gibbard, P. (2011) Quaternary glaciation. In: Singh, V.P., Singh, P. \& Haritashya, U.K. (Hrsg.) Encyclopedia of Snow, Ice and Glaciers, S. 873-882. Springer, Dordrecht, Niederlande.

Enigl, K., Matulla, C., Schlögl, M. \& Schmid, F. (2019) Derivation of canonical total-sequences triggering landslides and floodings in complex terrain. Advances in Water Resources 129, 178-188. DOI: https://doi.org/10.1016/j.advwatres.2019.04.018
Feser, F., Barcikowska, M., Krueger, O., Schenk, F., Weisse, R. \& Xia, L. (2015) Storminess over the North Atlantic and Northwestern Europe: a review. Quarterly Journal of the Royal Meteorological Society 141, 350-382. DOI: https://doi.org/10.1002/qj.2364.

Fischer, A., Olefs, M., \& Abermann, J. (2011) Glaciers, snow and ski tourism in Austria's changing climate. Annals of Glaciology 52(58), 89-96. DOI: https://doi.org/10.3189/172756411797252338

Fischer, A., Seiser, B., Stocker Waldhuber, M., Mitterer, C. \& Abermann, J. (2015) Tracing glacier changes in Austria from the Little Ice Age to the present using a lidar-based high-resolution glacier inventory in Austria. The Cryosphere 9(2), 753-766. DOI: https:// doi.org/10.5194/tc-9-753-2015

Fischer, A., Helfricht, K. \& Stocker-Waldhuber, M. (2016) Local reduction of decadal glacier thickness loss through mass balance management in ski resorts. The Cryosphere 10(6), 2941-2952. DOI: https:// doi.org/10.5194/tc-10-2941-2016

Fischer, E.M. \& Knutti, R. (2016) Observed heavy precipitation increase confirms theory and early models. Nature Climate Change 6, 986991. DOI: https://doi.org/10.1038/nclimate3110

Formayer, H. \& Fritz, A. (2017) Temperature dependency of hourly precipitation intensities - surface versus cloud layer temperature. International Journal of Climatology 37(1), 1-10. DOI: https://doi. org/10.1002/joc.4678

Formayer, H. \& Haas, P. (2011) Einfluss von Luftmasseneigenschaften auf die Schneefallgrenze in Österreich. In: Prettenthaler, F. \& Formayer, H. (Hrsg.) Tourismus im Klimawandel: Zur regionalwirtschaftlichen Bedeutung des Klimawandels für die österreichischen Tourismusgemeinden, S. 48-50. Studien zum Klimawandel in Österreich, Band 6. Verlag der Österreichischen Akademie der Wissenschaften (ÖAW), Wien.

Formayer, H., Reichl, J., Offenthaler, I., Nadeem, I., Schmidthaler, M., König, M. \& Laimighofer, J. (2018) Shifts in Weather Incidents Threatening reliability of the electricity distribution and transmission /economic performance due to Climate Change \& Opportunities For Foresight planning. Endbericht zum Projekt SWITCH-OFF, gefördert durch den Klima- und Energiefonds im Rahmen des Programms „Austrian Climate Research Programme“ (ACRP). Institut für Meteorologie, Universität für Bodenkultur Wien, Österreich.

Francis, J.A., Skific, N. \& Vavrus, S.J. (2018) North American weather regimes are becoming more persistent: is Arctic amplification a factor? Geophysical Research Letters 45(20), 11414-11422. DOI: https://doi.org/10.1029/2018GL080252

Frei, P., Kotlarski, S., Liniger, M. A., \& Schär, C (2018) Future snowfall in the Alps: projections based on the EURO-CORDEX regional climate models. The Cryosphere 12(1), 1-24. DOI: https://doi. org/10.5194/tc-12-1-2018

Ganopolski, A., Calov, R. \& Claussen, M. (2010) Simulation of the last glacial cycle with a coupled climate ice-sheet model of intermediate complexity. Climate of the Past 6(2), 229-244. DOI: https:// doi.org/10.5194/cp-6-229-2010

Geels, F.W. (2018) Sustainability transitions. In: Castree, N., Hulme, M. \& Proctor, J.D. (Hrsg.) Companion to Environmental Studies, S. 719-724. Routledge, Abingdon, Vereinigtes Königreich.

Gobiet, A., Kotlarski, S., Beniston, M., Heinrich, G., Rajczak, J. \& Stoffel, M. (2014) 21st century climate change in the European Alps: a review. Science of the Total Environment 493, 1138-1151. DOI: https://doi.org/10.1016/j.scitotenv.2013.07.050

Gobiet, A., Ulreich, K., Hofstätter, M., Podesser, A., Olefs, M., Vergeiner, J. \& Zenkl, G. (2017) Langfristige Entwicklung hochalpiner Wintertemperaturen. Zentralanstalt für Meteorologie und Geodynamik (ZAMG), Wien. Online unter: https://www.zamg.ac.at/cms/de/ dokumente/klima/dok_news/2017/gobiet_etal-2017-hochalpinewintertemperaturen/at_download/file (letzter Zugriff: 02.04.2020).

Gobiet, A., Kotlarski, S., Frei, P., Rajczak J., Verfaillie, D., Morin, S. \& Olefs, M. (2018) Klimawandel - Was bedeutet er für den Schnee in den Alpen? Berg und Steigen 105, 90-98. Online unter: http:// 
www.bergundsteigen.at/file.php/archiv/2018/4/90-98\%28klimawandel\%29.pdf (letzter Zugriff: 02.04.2020).

Grünewald, T., Wolfsperger, F. \& Lehning, M. (2018) Snow farming: conserving snow over the summer season. The Cryosphere 12(1), 385-400, DOI: https://doi.org/10.5194/tc-12-385-2018

Haeberli, W. \& Beniston, M. (1998) Climate change and its impacts on glaciers and permafrost in the Alps. Ambio 27(4), 258-265. Online unter: https://www.jstor.org/stable/4314732 (letzter Zugriff:02.04.2020).

Haimberger, L., Tavolato, C. \& Sperka, S. (2012) Homogenization of the global radiosonde temperature dataset through combined comparison with reanalysis background series and neighboring stations. Journal of Climate 25, 8108-8131. DOI: https://doi.org/10.1175/ JCLI-D-11-00668.1

Hanzer, F., Marke, T. \& Strasser, U. (2014) Distributed, explicit modelling of technical snow production for a ski area in the Schladming Region (Austrian Alps). Cold Regions Science and Technology 108, 113-124. DOI: https://doi.org/10.1016/j.coldregions.2014.08.003

Hartl, L., Fischer, A. \& Olefs, M. (2018) Analysis of past changes in wet bulb temperature in relation to snow making conditions based on long term observations Austria and Germany. Global and Planetary Change 167, 123-136. DOI: https://doi.org/10.1016/j. gloplacha.2018.05.011

Hartmann, D.L., Klein Tank, A.M.G., Rusticucci, M., Alexander, L.V., Brönnimann, S., Charabi, Y., Dentener, F.J., Dlugokencky, E.J., Easterling, D.R., Kaplan, A., Soden, B.J., Thorne, P.W., Wild, M. \& Zhai, P.M. (2013) Observations: Atmosphere and Surface. In: Stocker, T.F., Qin, D., Plattner, G.-K., Tignor, M., Allen, G.-K., Boschung, J., Nauels, A., Xia, Y., Bex, V. \& Midgley, P.M. (Hrsg.) Climate Change 2013: The Physical Science Basis. Contribution of Working Group I to the Fifth Assessment Report of the Intergovernmental Panel on Climate Change (IPCC), S. 159-254. Cambridge University Press, Cambridge, Vereinigtes Königreich und New York, NY, USA. Online unter: https://www.ipcc.ch/site/assets/ uploads/2017/09/WG1AR5_Chapter02_FINAL.pdf (letzter Zugriff: 02.04.2020).

Haslinger, K. \& Bartsch, A. (2016) Creating long-term gridded fields of reference evapotranspiration in Alpine terrain based on a recalibrated Hargreaves method. Hydrology and Earth System Sciences 20(3), 1211-1223. DOI: https://doi.org/10.5194/hess-20-1211-2016

Haslinger, K., Schöner, W. \& Anders, I. (2016) Future drought probabilities in the Greater Alpine Region Based on COSMO-CLM experiments: spatial patterns and driving forces. Meteorologische Zeitschrift 25 (2), 137-148. DOI: https://doi.org/10.1127/ metz/2015/0604

Haslinger, K., Holawe, F. \& Blöschl, G. (2018) Spatial characteristics of precipitation shortfalls in the greater Alpine Region: a data-based analysis from observations. Theoretical and Applied Climatology 136, 717-731. DOI: https://doi.org/10.1007/s00704-018-2506-5

Haslinger, K., Hofstätter, M., Kroisleitner, C., Schöner, W., Laaha, G., Holawe, F. \& Blöschl, G. (2019) Disentangling drivers of meteorological droughts in the European Greater Alpine Region during the last two centuries. Journal of Geophysical Research: Atmospheres 124(23), 12404-12425. DOI: https://doi.org/10.1029/2018JD029527

Helfricht, K., Huss, M., Fischer, A. \& Otto, J.C. (2019) Calibrated ice thickness estimate for all glaciers in Austria. Frontiers in Earth Science 7, 68. DOI: https://doi.org/10.3389/feart.2019.00068

Hiebl, J. \& Frei, C. (2018) Daily precipitation grids for Austria since 1961 - development and evaluation of a spatial dataset for hydroclimatic monitoring and modelling. Theoretical and Applied Climatology 132(1-2), 327-345. DOI: https://doi.org/10.1007/s00704017-2093-x

Hiebl, J. \& Hofstätter, M. (2012) No increasing multi-day regional temperature variability in Austria following climate warming. Climatic Change 113, 733-750. DOI: https://doi.org/10.1007/s10584-0110389-x
Hiebl, J. \& Schöner, W. (2018) Temperature inversions in Austria in a warming climate: changes in space and time. Meteorologische Zeitschrift 27(4), 309-323. DOI: https://doi.org/10.1127/metz/2018/0899

Hock, R. (2003) Temperature index melt modelling in mountain areas. Journal of Hydrology 282 (1-4), 104-115. DOI: https://doi. org/10.1016/S0022-1694(03)00257-9

Hofstätter, M. \& Formayer, H. (2011) Modellierung der Schneedecke unter Berücksichtigung maschineller Beschneiung. In: Prettenthaler, F. \& Formayer, H. (Hrsg.) Tourismus im Klimawandel: Zur regionalwirtschaftlichen Bedeutung des Klimawandels für die österreichischen Tourismusgemeinden, S. 41-47. Studien zum Klimawandel in Österreich, Band 6. Verlag der Österreichischen Akademie der Wissenschaften (ÖAW), Wien, Österreich.

Hofstätter, M., Lexer, A., Homann, M. \& Blöschl, G. (2018) Large-scale heavy precipitation over central Europe and the role of atmospheric cyclone track types. International Journal of Climatology 38 (Suppl. 1), 497-517. DOI: https://doi.org/10.1002/joc.5386.

Hohenwallner, D., Becsi, B., Formayer, H., Huber, H., Liehr, C., Prutsch, A. \& Zebisch, M (2018) Climate change impact maps for Austrian regions. Endbericht zum Projekt CLIMA-MAP, gefördert durch den Klima- und Energiefonds im Rahmen des Programms „Austrian Climate Research Programme" (ACRP).

Hurrell, J.W. (1995) Decadal Trends in the North Atlantic Oscillation: Regional Temperatures and Precipitation. Science 269(5224), 676679. DOI: https://doi.org/10.1126/science.269.5224.676

IPCC (2013) Summary for Policymakers. In: Stocker, T.F., Qin, D., Plattner, G.-K., Tignor, M., Allen, G.-K., Boschung, J., Nauels, A., Xia, Y., Bex, V. \& Midgley, P.M. (Hrsg.) Climate Change 2013: The Physical Science Basis. Contribution of Working Group I to the Fifth Assessment Report of the Intergovernmental Panel on Climate Change, S. 3-29. Cambridge University Press, Cambridge, Vereinigtes Königreich und New York, NY, USA. Online unter: https:// www.ipcc.ch/site/assets/uploads/2018/02/WG1AR5_SPM_FINAL. pdf (letzter Zugriff: 02.04.2020).

IPCC (2014) Climate Change 2014: Synthesis Report. Contribution of Working Groups I, II and III to the Fifth Assessment Report of the Intergovernmental Panel on Climate Change. Intergovernmental Panel on Climate Change (IPCC), Genf, Schweiz. Online unter: https:// www.ipcc.ch/site/assets/uploads/2018/02/SYR_AR5_FINAL_full. pdf (letzter Zugriff: 02.04.2020).

IPCC (2018) Global warming of $1.5^{\circ} \mathrm{C}$. An IPCC Special Report on the impacts of global warming of $1.5^{\circ} \mathrm{C}$ above pre-industrial levels and related global greenhouse gas emission pathways, in the context of strengthening the global response to the threat of climate change, sustainable development, and efforts to eradicate poverty. Intergovernmental Panel on Climate Change (IPCC), Genf, Schweiz. Online unter: https://www.ipcc.ch/site/assets/uploads/sites/2/2019/06/ SR15_Full_Report_Low_Res.pdf (letzter Zugriff: 02.04.2020).

IPCC (2019a) Climate Change and Land. An IPCC Special Report on climate change, desertification, land, degradation, sustainable land management, food security and greenhouse gas fluxes in terrestrial ecosystems: Summary for Policymakers. Intergovernmental Panel on Climate Change (IPCC), Genf, Schweiz. Online unter: https://www. ipcc.ch/site/assets/uploads/sites/4/2020/02/SPM_Updated-Jan20.pdf (letzter Zugriff: 02.04.2020).

IPCC (2019b) The Ocean and Cryosphere in a Changing Climate. An IPCC Special Report on the Ocean and Cryosphere in a Changing Climate: Summary for Policymakers. Intergovernmental Panel on Climate Change (IPCC), Genf, Schweiz. Online unter: https://www. ipcc.ch/site/assets/uploads/sites/3/2019/11/03_SROCC_SPM_FINAL.pdf (letzter Zugriff: 14.04.2020).

Ivancic, T.J \& Shaw, S.B. (2016) A U.S.-based analysis of the ability of the Clausius-Clapeyron relationship to explain changes in extreme rainfall with changing temperature. Journal of Geophysical Research: Atmospheres 121(7), 3066-3078. DOI: https://doi.org/ 10.1002/2015JD024288 
Jacob, D., Petersen, J., Eggert, B., Alias, A., Bøssing Christensen, O., Bouwer, L.M., Braun, A., Colette, A., Déqué, M., Georgievski, G., Georgopoulou, E., Gobiet, A., Menut, L., Nikulin, G., Haensler, A., Hempelmann, N., Jones, C., Keuler, K., Kovats, S., Kröner, N., Kotlarski, S., Kriegsmann, A., Martin, E., van Meijgaard, E., Moseley, C., Pfeifer, S., Preuschmann, S., Radermacher, C., Radtke, K., Rechid, D., Rounsevell, M., Samuelsson, P., Somot, S., Soussana, J.-F., Teichmann, C., Valentini, R., Vautard, R., Weber, B. \& Yiou, P. (2014) EURO-CORDEX: new high-resolution climate change projections for European impact research. Regional Environmental Change 14(2), 563-578. DOI: https://doi.org/10.1007/s10113-0130499-2

Kapsch, M.-L., Kunz, M., Vitolo, R. \& Economou, T. (2012) Longterm trends of hail-related weather types in an ensemble of regional climate models using a Bayesian approach. Journal of Geophysical Research: Atmospheres 117(D15107). DOI: https://doi.org/10.1029/ 2011JD017185

Kenner, R., Noetzli, J., Hoelzle, M., Raetzo, H. \& Phillips, M. (2019) Distinguishing ice-rich and ice-poor permafrost to map ground temperatures and ground ice occurrence in the Swiss Alps. The Cryosphere 13(7), 1925-1941. DOI: https://doi.org/10.5194/tc-131925-2019

Klein, G., Vitasse, Y., Rixen, C., Marty, C. \& Rebetez, M. (2016) Shorter snow cover duration since 1970in the Swiss Alps due to earlier snowmelt more than to later snow onset. Climatic Change 139(3-4), 637-649. DOI: https://doi.org/10.1007/s10584-016-1806-y

Kornhuber, K., Osprey, S., Coumou, D., Petri, S., Petoukhov, V., Rahmstorf, S. \& Gray, L. (2019) Extreme weather events in early summer 2018 connected by a recurrent hemispheric wave-7 pattern. Environmental Research Letters 14(5), 054002. DOI: https://doi. org/10.1088/1748-9326/ab13bf

Kotlarski, S., Gobiet, A., Frei, P., Morin, S., Rajczak, J., Scherrer, S. \& Verfaillie, D. (2018) Das Alpenklima im Wandel: Winterliche Schneefallmengen werden abnehmen, in tiefen Lagen fast komplettes Verschwinden der Schneedecke möglich. FdSnow 53, 38 45. Online unter: https://www.stiftung.ski/fdsnow/dflip/FdSnow.pdf (letzter Zugriff: 14.04.2020).

Kromp-Kolb, H. \& Formayer, H. (2018) + 2 Grad: warum wir uns für die Rettung der Welt erwärmen sollten. Molden, Wien und Graz, Österreich.

Kromp-Kolb, H., Nakicenovic, N., Seidl, R., Steininger, K., Ahrens, B., Auer, I., Baumgarten, A., Bednar-Friedl, B., Eitzinger, J., Foelsche, U., Formayer, H., Geitner, C., Glade, T., Gobiet, A., Grabherr, G., Haas, R., Haberl, H., Haimberger, L., Hitzenberger, R., König, M., Köppl, A., Lexer, M., Loibl, W., Molitor, R., Moshammer, H., Nachtnebel, H.-P., Prettenthaler, F., Rabitsch, W., Radunsky, K., Schneider, L., Schnitzer, H., Schöner, W., Schulz, N., Seibert, P., Stagl, S., Steiger, R., Stötter, H., Streicher, W. \& Winiwarter, W. (2014): Synthese. In: APCC (2014) Österreichischer Sachstandsbericht Klimawandel 2014 (AAR14), S. 67-131. Austrian Panel on Climate Change (APCC), Verlag der Österreichischen Akademie der Wissenschaften (ÖAW), Wien, Österreich. Online unter: http://www. austriaca.at/APCC_AAR2014.pdf (letzter Zugriff: 14.04.2020).

Landauer, M., Pröbstl, U. \& Haider, W. (2012) Managing cross-country skiing destinations under the conditions of climate change-Scenarios for destinations in Austria and Finland. Tourism Management 33(4), 741-751. DOI: https://doi.org/10.1016/j.tourman.2011.08.007

Landauer, M., Pröbstl, U. \& Haider, W. (2014) The influence of culture on climate change adaptation strategies: preferences of cross-country Skiers in Austria and Finland. Journal of Travel Research 53(1), 96-110. DOI: https://doi.org/10.1177/0047287513481276

Laternser, M. \& Schneebeli, M. (2003) Long-term snow climate trends of the Swiss Alps (1931-99). International Journal of Climatology 23(7), 733-750. DOI: https://doi.org/10.1002/joc.912

Leduc, M., Mailhot, A., Frigon, A., Martel, J.-L., Ludwig, R., Brietzke, G.B., Giguere, M., Brissette, F., Turcotte, R., Braun, M. \& Scinocca,
J. (2019) The ClimEx project: A 50-member ensemble of climate change projections at $12-\mathrm{km}$ resolution over Europe and Northeastern North America with the Canadian Regional Climate Model (CR'CM5). Journal of Applied Meteorology and Climatology 58(4), 663-693. DOI: https://doi.org/10.1175/JAMC-D-18-0021.1

Lehmann, J., Coumou, D. \& Frieler, K. (2015) Increased record-breaking precipitation events under global warming. Climatic Change 132(4), 501-515. DOI: https://doi.org/10.1007/s10584-015-1466-3

Lehning, M., Ebner, P.P. \& Rhyner, H. (2019) Schnee und Klimawandel im Hochgebirge: Prozessverständnis, Vorhersagen und Anpassungsmaßnahmen. FdSnow 53, 46-51. Online unter: https:/www.stiftung. ski/fdsnow/dflip/FdSnow.pdf (letzter Zugriff: 14.04.2020).

Lenderink, G. \& van Meijgaard, E. (2008) Increase in hourly precipitation extremes beyond expectations from temperature changes. Nature Geoscience 1(8), 511-514. DOI: https://doi.org/10.1038/ ngeo 262

Lenderink, G. \& van Meijgaard, E. (2010) Linking increases in hourly precipitation extremes to atmospheric temperature and moisture changes. Environmental Research Letters 5(2), 025208. DOI: https:// doi.org/10.1088/1748-9326/5/2/025208

Lise, W. \& Tol, R.S.J. (2002) Impact of climate on tourism demand. Climatic Change 55(4), 429-449. DOI: https://doi.org/ 10.1023/A:1020728021446

Lochbihler, K., Lenderink, G. \& Siebesma, A.P. (2019) Response of extreme precipitating cell structures to atmospheric warming. Journal of Geophysical Research: Atmospheres 124(13), 6904-6918. DOI: https://doi.org/10.1029/2018JD029954

Maddison, D. (2001) In search of warmer climates? The impact of climate change on flows of British tourists. Climatic Change 49(1-2), 193-208. DOI: https://doi.org/10.1023/A:1010742511380

Manara, V., Brunetti, M., Celozzi, A., Maugeri, M., Sanchez-Lorenzo, A. \& Wild, M. (2016) Detection of dimming/brightening in Italy from homogenized all-sky and clear-sky surface solar radiation records and underlying causes (1959-2013). Atmospheric Chemistry and Physics 16(17), 11145-11161. DOI: https://doi.org/10.5194/acp-1611145-2016

Mann, M. E., Rahmstorf, S., Kornhuber, K., Steinman, B.A., Miller, S.K. \& Coumou, D. (2017) Influence of anthropogenic climate change on planetary wave resonance and extreme weather events. Scientific Reports 7, 45242. DOI: https://doi.org/10.1038/srep45242

Marke, T., Hanzer, F., Olefs, M. \& Strasser, U. (2018) Simulation of past changes in the Austrian snow cover 1948-2009. Journal of Hydrometeorology 19(10), 1529-1545. DOI: https://doi.org/10.1175/ JHM-D-17-0245.1

Marsh, P.T., Brooks, H.E. \& Karoly, D.J. (2009) Preliminary investigation into the severe thunderstorm environment of Europe simulated by the Community Climate System Model 3. Atmospheric Research 93(1-3), 607-618. DOI: https://doi.org/10.1016/j.atmosres.2008.09.014

Marty, C. (2008) Regime shift of snow days in Switzerland. Geophysical Research Letters 35(12), L12501. DOI: https://doi.org/10.1029/ 2008GL033998

Marty, C., Tilg, A.-M. \& Jonas, T. (2017a) Recent evidence of largescale receding snow water equivalents in the European Alps. Journal of Hydrometeorology 18(4), 1021-1031. DOI: https://doi.org/10.1175/ JHM-D-16-0188.1

Marty, C., Schlögl, S., Bavay, M. \& Lehning, M. (2017b) How much can we save? Impact of different emission scenarios on future snow cover in the Alps. The Cryosphere 11(1), 517-529. DOI: https://doi. org/10.5194/tc-11-517-2017

Matulla, C., Schöner, W., Alexandersson, H., von Storch, H. \& Wang, X.L. (2008) European storminess: late nineteenth century to present. Climate Dynamics 31(2-3), 125-130. DOI: https://doi.org/10.1007/ s00382-007-0333-y

Matulla, C., Tordai, J., Schlögl, M., Ganekind, M., Matulla, H., Ressl, H. \& Chimani, B. (2018a) Establishment of a long-term lake-sur- 
face temperature dataset within the European Alps extending back to 1880. Climate Dynamics 52(9-10), 5673-5689. DOI: https://doi. org $/ 10.1007 / \mathrm{s} 00382-018-4479-6$

Matulla, C., Hollósi, B., Andre, K., Gringinger, J., Chimani, B., Namyslo, J., Fuchs, T., Auerbach, M., Herrmann, C., Sladek, B., Berghold, H., Gschier, R. \& Eichinger-Vill, E. (2018b) Climate change driven evolution of hazards to Europe's transport infrastructure throughout the twenty-first century. Theoretical and Applied Climatology 133(1-2), 227-242. DOI: https://doi.org/10.1007/s00704017-2127-4

Matulla, C., Feser, F., Tordai, J., Schlögl, M., Starke, H., Schöner, W., Chimani, B., Hofstätter, M., Brazdil, R. \& Andre, K. (2020) Kapitel Stürme. In: Glade, T., Mergili, M. \& Sattler, K. (Hrsg.) ExtremA 2019: Aktueller Wissensstand zu Extremereignissen alpiner Naturgefahren in Österreich, S. 123-144. V\&R unipress, Wien, Österreich.

Matzarakis, A. (2007) Climate, thermal comfort and tourism. In: Amelung, B., Blazejczyk, K. \& Matzarakis, A. (Hrsg.) Climate change and tourism - assessment and coping strategies, S. 139-154. Institute of Geography and Spatial Organization, Polish Academy of Sciences, Warschau, Polen.

Mieczkowski, Z.(1985) The tourism climate index: a method for evaluating world climates for tourism. The Canadian Geographer/ Le Géographe canadién 29(3), 220-233. DOI: https://doi.org/ 10.1111/j.1541-0064.1985.tb00365.x

Milankovitch, M. (1941) Kanon der Erdbestrahlung und seine Anwendung auf das Eiszeitenproblem. Reprint in englischer Sprache (1998): Canon of insolation and the ice-Age problem. Königlich Serbische Akademie, Belgrad, Serbien.

Mohr, S., Kunz, M. \& Keuler, K. (2015) Development and application of a logistic model to estimate the past and future hail potential in Germany. Journal of Geophysical Research 120(9), 3939-3956. DOI: https://doi.org/10.1002/2014JD022959

Nabat, P., Somot, S., Mallet, M., Sanchez-Lorenzo, A. \& Wild, M. (2014) Contribution of anthropogenic sulfate aerosols to the changing EuroMediterranean climate since 1980. Geophysical Research Letters 41 (15), 5605-5611. DOI: https://doi.org/10.1002/2014GL060798

NCCS (2018) CH2018 - Climate Scenarios for Switzerland, Technical Report. National Centre for Climate Services (NCCS), Zürich, Schweiz. Online unter: https://naturwissenschaften.ch/uuid/9081 ee9e-9a07-5ffc-a720-4031 feb879b1?r=$20190807115818 \quad 1565138729$ 947a7370-149b-5b30-afd6a22c38db1c60 (letzter Zugriff: 01.04.2020).

Nemec, J., Gruber, C., Chimani, B. \& Auer, I. (2013) Trends in extreme temperature indices in Austria based on a new homogenised dataset. International Journal of Climatology 33(6), 1538-1550. DOI: https://doi.org/10.1002/joc.3532

Nicholls, S. (2006) Climate change, tourism and outdoor recreation in Europe. Managing Leisure 11(3), 151-163. DOI: https://doi. org/10.1080/13606710600715226

Noetzli, J., Christiansen, H.H., Gugliemin, M., Romanovsky, V.E., Shiklomanov, N.I., Smith, S.L. \& Zhao, L. (2016) Permafrost thermal states [in "State of the Climate in 2015"]. Bulletin of the American Meteorological Society 97(8), 20-21. DOI: https://doi.org/10.1175/ 2016BAMSStateoftheClimate. 1

Olefs, M. \& Obleitner, F. (2007) Numerical simulations on artificial reduction of snow and ice ablation. Water Resources Research 43(6), W06405. DOI: https://doi.org/10.1029/2006WR005065

Olefs, M. \& Fischer, A. (2008) Comparative study of technical measures to reduce snow and ice ablation in Alpine glacier ski resorts. Cold Regions Science and Technology 52(3), 371-384. DOI: https://doi. org/10.1016/j.coldregions.2007.04.021

Olefs, M., Kuhn, M. \& Fischer, A. (2009) The effect of climate change on the runoff behaviour of glacierised Alpine catchments with regard to reservoir power stations. Geophysical Research Abstracts 11, EGU2009-4813. EGU General Assembly 2009. Online unter:
https://meetingorganizer.copernicus.org/EGU2009/EGU2009-4813. pdf (letzter Zugriff: 15.04.2020).

Olefs, M. \& Lehning, M. (2010) Textile protection of snow and ice: measured and simulated effects on the energy and mass balance. Cold Regions Science and Technology 62(2-3), 126-41. DOI: https:// doi.org/10.1016/j.coldregions.2010.03.011

Olefs, M., Fischer, A. \& Lang, J. (2010) Boundary conditions for artificial snow production in the Austrian Alps. Journal of Applied Meteorology and Climatology 49(6), 1096-1113. DOI: https://doi. org/10.1175/2010JAMC2251.1

Olefs, M., Girstmair, A., Hiebl, J., Koch, R. \& Schöner, W. (2017) An area-wide snow climatology for Austria since 1961 based on newly available daily precipitation and air temperature grids. Geophysical Research Abstracts 19, EGU2017-12249. EGU General Assembly 2017. Online unter: https://meetingorganizer.copernicus.org/ EGU2017/EGU2017-12249.pdf (letzter Zugriff: 15.04.2020).

Olefs, M., Koch, R. \& Gobiet, A. (2019) Klima und Schnee in Österreich: beobachtete Vergangenheit und erwartete Zukunft. FdSnow 53, 28-37. Online unter: https://www.stiftung.ski/fdsnow/dflip/FdSnow. pdf (letzter Zugrifff: 15.04.2020).

Pfeifroth, U., Bojanowski, J.S., Clerbaux, N., Manara, V., Sanchez-Lorenzo, A., Trentmann, J., Walawender, J.P. \& Hollmann, R. (2018) Satellite-based trends of solar radiation and cloud parameters in Europe. Advances in Science and Research 15, 31-37. DOI: https:// doi.org/10.5194/asr-15-31-2018

Pistotnik, G., Hofstätter, M. \& Lexer, A. (2020) Kapitel Starkniederschlag und Hagel. In: Glade, T., Mergili, M. \& Sattler, K. (Hrsg.) ExtremA 2019: Aktueller Wissensstand zu Extremereignissen alpiner Naturgefahren in Österreich, S. 145-176. V\&R unipress, Wien, Österreich.

Pomeroy, J.W. \& Brun, E. (2001) Physical properties of snow. In: Jones, H.G. (Hrsg.) Snow ecology: an interdisciplinary examination of snow-covered ecosystems, S. 45-126. Cambridge University Press, Cambridge, Vereinigtes Königreich und New York, NY, USA.

Prettenthaler F. \& Formayer, H. (2011) Tourismus im Klimawandel: Zur regionalwirtschaftlichen Bedeutung des Klimawandels für die österreichischen Tourismusgemeinden. Studien zum Klimawandel in Österreich, Band 6. Verlag der Österreichischen Akademie der Wissenschaften (ÖAW), Wien, Österreich.

Pröbstl, U. \& Damm, B. (2009) Wahrnehmung und Bewertung von Naturgefahren als Folge von Gletscherschwund und Permafrostdegradation in Tourismus: Destinationen am Beispiel des Tuxer Tals (ZillertalerAlpen/Österreich). Endbericht von Start-Clim2008.F in StartClim2008: Anpassung an den Klimawandel in Österreich. Online unter: http://www.startclim.at/fileadmin/user_upload/reports/ StC108F.pdf (letzter Zugriff: 15.04.2020).

Púčik, T., Groenemeijer, P., Rädler, A.T., Tijssen, L., Nikulin, G., Prein, A. F., van Meijgaard, E., Fealyh, R., Jacob, D. \& Teichmann, C. (2017) Future changes in European severe convection environments in a regional climate model ensemble. Journal of Climate 30(17), 6771-6794. DOI: https://doi.org/10.1175/JCLI-D-16-0777.1

Rädler, A.T., Groenemeijer, P., Faust, E. \& Sausen, R. (2018) Detecting severe weather trends using an additive regressive convective hazard model (AR-CHaMo). Journal of Applied Meteorology and Climatology 57(3), 569-87. DOI: https://doi.org/10.1175/ JAMC-D-17-0132.1

Rafelsberger, P. (2007) Phänologische, klimatologische Untersuchungen einer Thermikwolke. Diplomarbeit, Institut für Meteorologie und Geophysik, Universität Innsbruck, Österreich.

Reniu, M.G. (2017) Evapotranspiration projections in Austria under different climate change scenarios. Masterarbeit am Institut für Meteorologie und Klimatologie (BOKU-Met), Universität für Bodenkultur Wien, Österreich. Online unter: https://abstracts.boku.ac.at/ download.php?dataset_id=17712\&property_id=107\&role_id=NONE (letzter Zugriff: $\overline{15}$.04.2020). 
Richardson, R.B. \& Loomis, J.B. (2004) Adaptive recreation planning and climate change: a contingent visitation approach. Ecological Economics 50(1-2), 83-99. DOI: https://doi.org/10.1016/j.ecolecon.2004.02.010

Rogelj, J., Popp, A., Calvin, K.V., Luderer, G., Emmerling, J., Gernaat, D., Fujimori, S., Strefler, J., Hasegawa, T., Marangoni, G., Krey, V., Kriegler, E., Riahi, K., van Vuuren, D.P., Doelman, J., Drouet, L., Edmonds, J., Fricko, O., Harmsen, M., Havlík, P., Humpenöder, F., Stehfest, E. \& Tavoni, M. (2018) Scenarios towards limiting global mean temperature increase below 1.5 C. Nature Climate Change 8(4), 325-332. DOI: https://doi.org/10.1038/s41558-018-0091-3

Romanowsky, E., Handorf, D., Jaiser, R., Wohltmann, I., Dorn, W., Ukita, J., Cohen, J., Dethloff, K. \& Rex, M. (2019) The role of stratospheric ozone for Arctic-midlatitude linkages. Scientific Reports 9, 7962. DOI: https://doi.org/10.1038/s41598-019-43823-1

Roth, R. (Hrsg.) (2018) Gemeinsames Positionspapier Expertenforum 2018: Klima.Schnee.Sport. FdSnow 53, 6-7. Online unter: https://www.stiftung.ski/fdsnow/dflip/FdSnow.pdf (letzter Zugrifff: 15.04.2020).

Saffioti, C., Fischer, E.M., Scherrer, S.C. \& Knutti, R. (2016) Reconciling observed and modeled temperature and precipitation trends over Europe by adjusting for circulation variability. Geophysical Research Letters 43(15), 8189-8198. DOI: https://doi.org/10.1002/ 2016GL069802

Sander, J. (2011) Extremwetterereignisse im Klimawandel: Bewertung der derzeitigen und zukünftigen Gefährdung. Dissertation, Ludwig Maximilians Universität München, Deutschland. Online unter: https://core.ac.uk/download/pdf/11032462.pdf (letzter Zugriff: 15.04.2020)

Sass O., Malowerschnig, B., Vacik, H., Arpaci, A., Müller, M., Formayer, H., Leidinger, D. \& Sailer, R. (2014) Fire risk and vulnerability of Austrian forests under the impact of climate change. Endbericht zum Projekt FIRIA, gefördert durch den Klima- und Energiefonds im Rahmen des Programms „Austrian Climate Research Programme“ (ACRP). Online unter: https://www.klimafonds. gv.at/wp-content/uploads/sites/6/01112014FIRIASASSEBACRP3B068712K10AC1K00091.pdf (letzter Zugriff: 15.04.2020).

Scherrer, S.C. \& Begert, M. (2019) Effects of large-scale atmospheric flow and sunshine duration on the evolution of minimum and maximum temperature in Switzerland. Theoretical and Applied Climatology 138(1-2), 227-235. DOI: https://doi.org/10.1007/ s00704-019-02823-x

Scherrer, S.C., Appenzeller, C. \& Laternser, M. (2004) Trends in Swiss Alpine snow days: the role of local- and large-scale climate variability. Geophysical Research Letters 31(13), L13215. DOI: https://doi. org/10.1029/2004GL020255

Schlögl, M. \& Matulla, C. (2018) Potential future exposure of European land transport infrastructure to rainfall-induced landslides throughout the 21st century. Natural Hazards and Earth System Sciences 18(4), 1121-1132. DOI: https://doi.org/10.5194/nhess-18-1121-2018

Schmucki, E., Marty, C., Fierz, C. \& Lehning, M. (2015). Simulations of 21 st century snow response to climate change in Switzerland from a set of RCMs. International Journal of Climatology 35(11), 3262-3273. DOI: https://doi.org/10.1002/joc.4205

Schöner, W., Auer, I. \& Böhm, R. (2009) Long term trend of snow depth at Sonnblick (Austrian Alps) and its relation to climate change. Hydrological Processes 23(7), 1052-1063. DOI: https:// doi.org/10.1002/hyp.7209

Schöner, W., Böckli, L., Hausmann, H., Otto, J.-C., Reisenhofer, S., Riedl, C. \& Seren, S. (2012) Patterns of permafrost at Hoher Sonnblick (Austrian Alps) - Extensive field-measurements and modelling approaches. Austrian Journal of Earth Sciences 105(2), 154-168. Online unter: https://epic.awi.de/id/eprint/35601/1/schoener_et_al_ ajes_105_2.pdf (letzter Zugriff: 15.04.2020).

Schöner, W., Koch, R., Matulla, C., Marty, C. \& Tilg, A.-M. (2018) Spatiotemporal patterns of snow depth within the Swiss-Austrian
Alps for the past half century (1961 to 2012) and linkages to climate change. International Journal of Climatology 39(3), 1589-1603. DOI: https://doi.org/10.1002/joc.5902

Schroeer, K. \& Kirchengast, G. (2018) Sensitivity of extreme precipitation to temperature: the variability of scaling factors from a regional to local perspective. Climate Dynamics 50(11-12), 3981-3994. DOI: https://doi.org/10.1007/s00382-017-3857-9

Schwaiger, H., Bird, D.N., Damm, A., Kortschak, D. \& Prettenthaler, F. (2017) Die Klima- und Energiebilanz von Skigebieten mit technischer Beschneiung unter Berücksichtigung des Albedo-Effektes. Joanneum Research Forschungsgesellschaft mbH, LIFE - Zentrum für Klima, Energie und Gesellschaft, Graz, Österreich. Online unter: https://www.joanneum.at/fileadmin/LIFE/News_Bilder_Logos/ news/Klima-und_Energiebilanz_von_Beschneiung.pdf (letzter Zugriff: 15.04 .2020$)$.

Scott, D., McBoyle, G. \& Schwartzentruber, M. (2004) Climate change and the distribution of climatic resources for tourism in North America. Climate Research 27(2), 105-117. DOI: https://doi.org/10.3354/ cr027105

Scott, D., Rutty, M., Amelung, B. \& Tang, M. (2016) An inter-comparison of the holiday climate index (HCI) and the tourism climate index (TCI) in Europe. Atmosphere 7(6), 80. DOI: https://doi.org/10.3390/ atmos 7060080

Serquet, G., Marty, C., Dulex, J.P. \& Rebetez, M. (2011) Seasonal trends and temperature dependence of the snowfall/precipitation-day ratio in Switzerland. Geophysical Research Letters 38(7), L07703. DOI: https://doi.org/10.1029/2011GL046976

Shakun, J.D., Clark, P.U., He, F., Marcott, S.A., Mix, A.C., Liu, Z., Otto-Bliesner, B., Schmittner, A. \& Bard, E. (2012) Global warming preceded by increasing carbon dioxide concentrations during the last deglaciation. Nature 484(7392), 49-54. DOI: https://doi. org/10.1038/nature10915

Spandre, P., François, H., Morin, S., George-Marcelpoil, E. (2015) Snowmaking in the French Alps: climatic context, existing facilities and outlook. Journal of Alpine Research 103(2), 1-17. DOI: https:// doi.org/10.4000/rga.2913

Spandre, P., François, H., Verfaillie, D., Lafaysse, M., Déqué, M., Eckert, N., George, E. \& Morin, S. (2019) Climate controls on snow reliability in French Alps ski resorts. Scientific Reports 9, 8043. DOI: https://doi.org/10.1038/s41598-019-44068-8

Steiger, R. (2016) Klimawandel und Naturtourismus. In: Mayer, M. \& Job, H. (Hrsg.) Naturtourismus: Chancen und Herausforderungen, S. 49-60. Studien zur Freizeit- und Tourismusforschung, Band 12. Verlag MetaGIS-Systems, Mannheim, Deutschland.

Steiger, R. \& Abegg, B. (2013) The sensitivity of Austrian ski areas to climate change. Tourism Planning \& Development 10(4), 480-493. DOI: https://doi.org/10.1080/21568316.2013.804431

Stephenson, D., Hannachi, A. \& O'Neill, A. (2004) On the existence of multiple climate regimes. Quarterly Journal of the Royal Meteorological Society 130(597), 583-605. DOI: https://doi.org/10.1256/ qj.02.146

Switanek, M.B., Troch, P.A., Castro, C.L., Leuprecht, A., Chang, H.-I., Mukherjee, R. \& Demaria, E.M.C. (2017) Scaled distribution mapping: a bias correction method that preserves raw climate model projected changes. Hydrology and Earth System Sciences 21(6), 2649-2666. DOI: https://doi.org/10.5194/hess-21-2649-2017

Trimmel, H., Weihs, P., Faroux, S., Formayer, H., Hamer, P., Hasel, K., Laimighofer, J., Leidinger, D., Masson, V., Nadeem, I., Oswald, S., Revesz, M. \& Schoetter, R. (2019) Thermal conditions during heat waves of a mid-European metropolis under consideration of climate change, urban development scenarios and resilience measures for the mid-21st century. Meteorologische Zeitschrift. PrePub DOI: https:// doi.org/10.1127/metz/2019/0966

Trnka, M., Balek, J., Štěpánek, P., Zahradníček, P., Možný, M., Eitzinger, J., Žalud, Z., Formayer, H., Turňa, M., Nejedlík, P., Semerádová, D., Hlavinka, P. \& Brázdil, R. (2016) Drought trends over part of 
Central Europe between 1961 and 2014. Climate Research 70(2-3), 143-160. DOI: https://doi.org/10.3354/cr01420

Tzedakis, P.C., Channell, J.E.T., Hodell, D.A., Kleiven, H.F. \& Skinner, L.C. (2012) Determining the natural length of the current interglacial. Nature Geoscience 5, 138-141. DOI: https://doi.org/10.1038/ ngeo 1358

UNFCCC (2015) The Paris Agreement. United Nations Framework Convention on Climate Change (UNFCCC), Bonn, Deutschland. Online unter: https://unfccc.int/files/essential_background/convention/application/pdf/english paris agreement.pdf (letzter Zugriff: 16.04.2020).

van Vuuren, D.P., Edmonds, J., Kainuma, M., Riahi, K., Thomson, A., Hibbard, K., Hurtt, G.C., Kram, T., Krey, V., Lamarque, J.-F., Masui, T., Meinshausen, M., Nakicenovic, N., Smith, S.J. \& Rose, S.K. (2011) The representative concentration pathways: an overview. Climatic Change 109(1-2), 5. DOI: https://doi.org/10.1007/ s10584-011-0148-z

Walker, M., Johnsen, S., Rasmussen, S.O., Popp, T., Steffensen, J.-P., Gibbard, P., Hoek, W., Lowe, J., Andrews, J., Björck, S., Cwynar, L.C., Hughen, K., Kershaw, P., Kromer, B., Litt, T., Lowe, D.J., Nakagawa, T., Newnham, R. \& Schwander, J. (2009) Formal definition and dating of the GSSP (Global Stratotype Section and Point) for the base of the Holocene using the Greenland NGRIP ice core, and selected auxiliary records. Journal of Quaternary Science 24(1), 3-17. DOI: https://doi.org/10.1002/jqs. 1227

Waters, C.N., Zalasiewicz, J., Summerhayes, C., Barnosky, A.D., Poirier, C., Gałuszka, A., Cearreta, A. , Edgeworth, M., Ellis, E.C., Ellis, M., Jeandel, C., Leinfelder, R., McNeill, J.R., Richter, D. deB., Steffen, W., Syvitski, J., Vidas, D., Wagreich, M., Williams, M., Zhisheng, A., Grinevald, J., Odada, E., Oreskes, N. \& Wolfe, A.P. (2016) The Anthropocene is functionally and stratigraphically distinct from the Holocene. Science 351(6269), aad2622. DOI: https:// doi.org/10.1126/science.aad2622

Weihs, P. \& Laimighofer, J. (2019) SnowAlb - Effekte künstlicher Beschneiung auf den Strahlungshaushalt der Skiregion Saalbach-Hinterglemm. Endbericht von Start-Clim2018.C in StartClim2018: Synergien und Nutzungskonflikte bei der Umsetzung von Klimawandelanpassung und den Sustainable Development Goals in Österreich aus Sicht der Klimaforschung. Online unter: http://www.startclim. at/fileadmin/user_upload/StartClim2018_reports/StCl18C_lang.pdf (letzter Zugriff: 16.04.2020).
Wild, M. (2009) Global dimming and brightening: a review. Journal of Geophysical Research 114(D10), D00D16. DOI: https://doi.org/ 10.1029/2008JD011470

WMO (1970) The Beaufort scale of wind force (technical and operational aspects). Report submitted by the President of the Commission for Maritime Meteorology to the WMO Executive Committee at its twenty-second session. Report on marine science affairs (MSA) No. 3. World Meteorolgical Organization (WMO), Genf, Schweiz.

Zachos, J., Pagani, M., Sloan, L., Thomas, E. \& Billups, K. (2001) Trends, rhythms, and aberrations in global climate 65 Ma to present. Science 292(5517), 686-693. DOI: https://doi.org/10.1126/ science. 1059412

ZAMG (2019) HISTALP Langzeitklimareihen - Österreich: Sommerbericht 2019. Online unter: http://www.zamg.ac.at/histalp/download/ newsletter/HISTALP_AT_Sommernewsletter_2019.pdf (letzter Zugriff: 16.04.2020).

ZAMG (2020a) HISTALP Langzeitklimareihen - Österreich: Winterbericht 2019/20. Online unter: http://www.zamg.ac.at/histalp/ download/newsletter/HISTALP_AT_Winterbericht_2019_2020.pdf (letzter Zugriff: 16.04.2020).

ZAMG (2020b) HISTALP Langzeitklimareihen - Österreich: Jahresbericht 2019. Online unter: http://www.zamg.ac.at/histalp/download/ newsletter/HISTALP AT Jahresnewsletter 2019.pdf (letzter Zugriff: 16.04.2020).

Zampieri, M., Scoccimarro, E. \& Gualdi, S. (2013) Atlantic influence on spring snowfall over the Alps in the past 150 years. Environmental Research Letters 8(3), 034026. DOI: https://doi.org/10.1088/17489326/8/3/034026

Zebisch, M., Vaccaro, R., Niedrist, G., Schneiderbauer, S., Streifeneder, T., Weiß, M., Troi, A., Renner, K., Pedoth, L., Baumgartner, B. \& Bergonzi, V. (Hrsg.) (2018) Klimareport Südtirol 2018. Eurac Research, Bozen, Italien. Online unter: http://www.eurac.edu/de/research/mountains/remsen/projects/Documents/klimareport/Klimareport\%202018\%20DE_new.pdf (letzter Zugriff: 16.04.2020).

Žuvela-Aloise, M., Andre, K., Schwaiger, H., Bird, D.N. \& Gallaun, H. (2018) Modelling reduction of urban heat load in Vienna by modifying surface properties of roofs. Theoretical and Applied Climatology 131(3-4), 1005-1018. DOI: https://doi.org/10.1007/ s00704-016-2024-2

Open Access Dieses Buch wird unter der Creative Commons Namensnennung 4.0 International Lizenz (http://creativecommons.org/licenses/by/4.0/deed.de) veröffentlicht, welche die Nutzung, Vervielfältigung, Bearbeitung, Verbreitung und Wiedergabe in jeglichem Medium und Format erlaubt, sofern Sie den/die ursprünglichen Autor(en) und die Quelle ordnungsgemäß nennen, einen Link zur Creative Commons Lizenz beifügen und angeben, ob Änderungen vorgenommen wurden.

Die in diesem Buch enthaltenen Bilder und sonstiges Drittmaterial unterliegen ebenfalls der genannten Creative Commons Lizenz, sofern sich aus der Abbildungslegende nichts anderes ergibt. Sofern das betreffende Material nicht unter der genannten Creative Commons Lizenz steht und die betreffende Handlung nicht nach gesetzlichen Vorschriften erlaubt ist, ist für die oben aufgeführten Weiterverwendungen des Materials die Einwilligung des jeweiligen Rechteinhabers einzuholen. 